Aus der Abteilung Augenheilkunde

(Prof. Dr. med. H. Hoerauf)

im Zentrum Augenheilkunde und Hals - Nasen - Ohrenheilkunde der Medizinischen Fakultät der Universität Göttingen

\title{
Muskuloskeletale Beschwerden an der Wirbelsäule bei Augenärzten
}

\author{
INAUGURAL - DISSERTATION \\ zur \\ Erlangung des Doktorgrades \\ der Medizinischen Fakultät \\ der Georg - August - Universität zu Göttingen
}

\author{
vorgelegt von \\ Silke Eichberg \\ aus \\ Rostock - Warnemünde
}

Göttingen 2013 
Dekan: Prof. Dr. rer. nat. H. K. Kroemer

I. Berichterstatter: Prof. Dr. med. H. Hoerauf

II. Berichterstatter: PD Straube, BM BCh, MA, DPhil

III. Berichterstatter: Prof. Dr. med. dent. Mausberg

Tag der mündlichen Prüfung: 28.08.2013 


\section{Inhaltsverzeichnis}

$\begin{array}{ll}\text { Abkürzungsverzeichnis } & 4\end{array}$

1. Einleitung

1.1. Bedeutung von muskuloskeletalen Beschwerden bei Augenärzten 5

1.2. Fehlhaltung bei augenärztlichen Tätigkeiten 5

$\begin{array}{ll}\text { 1.3. } & \text { Aktuelle Datenlage }\end{array}$

$\begin{array}{lr}\text { 1.4. Pilot - Fragebogen und Auswertung } & 10\end{array}$

$\begin{array}{ll}\text { 1.5. Zielsetzung und Fragestellung } & 14\end{array}$

2. Material und Methoden

$\begin{array}{lr}\text { 2.1. } & \text { Fragebogen - Erstellung } \\ \end{array}$

$\begin{array}{ll}\text { 2.2. Erstellung des BVA - Umfragebogens } & 15\end{array}$

$\begin{array}{ll}\text { 2.2.1. Konsequenzen aus dem Pilot - Fragebogen und Änderungen } & 15\end{array}$

$\begin{array}{ll}\text { 2.2.2. Gliederung und Fragenkomplexe } & 16\end{array}$

$\begin{array}{lr}\text { 2.2.3. Probelauf } & 16\end{array}$

\begin{tabular}{lr} 
2.3. & Logistische Vorbereitung der BVA - Umfrage \\
\hline
\end{tabular}

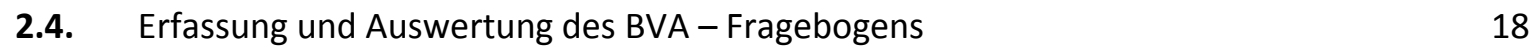

3. Ergebnisse

\begin{tabular}{lr} 
3.1. & Fragebogen - Rücklaufquote \\
\hline
\end{tabular}

$\begin{array}{lr}\text { 3.2. Kollektivbeschreibung } & 20\end{array}$ 
3.2.1. Ergebnisse zu Zielgrößen

3.2.1.1. Zielgröße „Vorschäden“ unter den Augenärzten/innen

3.2.1.2. Zielgröße „aktuelle Beschwerden“ unter den Augenärzten/innen

3.2.2. Zielgröße „aktuelle Beschwerden“ und Einflußgrößen (Spezialgebiet,

Tätigkeitsschwerpunkte, Sport) beeinflusst durch Alter und Geschlecht

3.2.3. Zielgröße „aktuelle Beschwerden“ der Augenärzte/innen beeinflusst durch

die Berufsjahre und deren Auswirkungen auf die Symptome und Lokalisationen der Beschwerden

3.2.4. Zielgröße „aktuelle Beschwerden“ der Augenärzte/innen beeinflusst durch die Tätigkeitsschwerpunkte „konservativ“, „operativ“ und Kombination von „konservativ“ und „operativ“ und deren Auswirkungen auf die Symptome und Lokalisationen der Beschwerden

3.3. Darstellung der einzelnen Tätigkeiten des Augenarztes und deren Auswirkungen auf die Zielgröße aktuelle Beschwerden unter den Augenärzten/innen

3.4. Behandlungen, denen sich die Augenärzte/innen mit aktuellen Beschwerden am muskuloskeletalen Bewegungsapparat unterzogen

3.5. Angaben aller Augenärzte/innen zur Intensität der Schmerzen/Beschwerden am muskuloskeletalen Bewegungsapparat

3.6. Angaben zur Optimierung des Arbeitsplatzes in der Augenheilkunde

\section{Diskussion}

4.1. Vergleich der Ergebnisse der Erhebung unter den Teilnehmern des BVA zu epidemiologischen Daten aus der Normalbevölkerung

4.2. Häufigkeit und Einfluss von Vorschäden auf bestehende Beschwerdelokalisationen 
4.4. Relevanz der Häufigkeit und Lokalisation der aktuellen Beschwerden in den einzelnen Tätigkeitsschwerpunkten

4.5. Optimierung des Arbeitsplatzes

5. Zusammenfassung

6. Anhang: Fragebögen

7. Literaturverzeichnis 


\section{Abkürzungsverzeichnis}

$\begin{array}{ll}\text { AAD } & \text { Augenärztliche Akademie Deutschland } \\ \text { BKK } & \text { Betriebskrankenkassen } \\ \text { BWS } & \text { Brustwirbelsäule } \\ \text { BV } & \text { Bundesverband } \\ \text { BVA } & \text { Berufsverband der Augenärzte Deutschland e.V. } \\ \text { HWS } & \text { Halswirbelsäule } \\ \text { ISG } & \text { lleosakralgelenk } \\ \text { LWS } & \text { Lendenwirbelsäule } \\ \text { MSDs } & \text { Musculoskeletal disorders } \\ \text { N } & \text { Newton } \\ \text { NAS } & \text { Numerische Analogskala } \\ \text { NSAID } & \text { nonsteroidal anti - inflammatory drug } \\ \text { RKI } & \text { Robert Koch Institut } \\ \text { TKK } & \text { Techniker Krankenkasse } \\ \text { UMG } & \text { Universitätsmedizin Göttingen }\end{array}$




\section{Einleitung}

\subsection{Bedeutung von muskuloskeletalen Beschwerden bei Augenärzten}

Muskuloskeletale Erkrankungen (Musculoskeletal disorders $=$ MSDs) im Rumpf- und Nackenbereich, sowie auch oberen Extremitäten sind eine Zivilisationskrankheit mit hoher Morbidität und enormen sozioökonomischen Konsequenzen. So haben vier von fünf Erwachsenen im Laufe Ihres Lebens ein- oder mehrmals Rückenschmerzen. (TKK 1997) Im Rahmen der routinemäßigen augenärztlichen Untersuchung kommt es zu einer starken und chronischen Mehrbelastung der Wirbelsäule und der oberen Extremitäten mit daraus möglicherweise resultierender höherer Inzidenz und Prävalenz als in der Durchschnittsbevölkerung. Die Ursache für die einseitige und übermäßige Beanspruchung der Muskulatur und Gelenke an der Wirbelsäule und der oberen Extremitäten, besteht in dem regelmäßigen Wiederholen von Arbeitsabläufen mit instabiler und unphysiologischer Haltung. Hierzu kommt ein zum Teil sehr hohes Arbeitsaufkommen durch stetig steigende Patientenzahlen sowie ein dadurch bedingter Mangel an Entspannungspausen.

Die Wirbelsäule des menschlichen Körpers kompensiert durch ihre doppelte $S$ - Form extreme Belastungen. Dabei wirkt die Krümmung wie eine Spiralfeder, indem, sie Erschütterungen und Belastungen gleichmäßig auf den Körper verteilt. Die umgebende Rumpfmuskulatur sowie die Bänder und die zwischen den einzelnen Wirbeln liegenden Bandscheiben dienen zur Stabilisierung und Flexibilität der Wirbelsäule. Neben altersbedingten Veränderungen unterliegt die Wirbelsäule den beschriebenen belastungsbedingten Umgestaltungen, die zum Beispiel durch langes falsches Sitzen und Stehen zur frühzeitigen Entstehung chronischer Schäden führen.

\subsection{Fehlhaltung bei augenärztlichen Tätigkeiten}

Die langandauernde Arbeit in einer bestimmten Haltung, häufig unter ergonomisch schlechten Bedingungen, wie beispielsweise an der Spaltlampe (s. Abb.1) oder das lange Sitzen am OP-Mikroskop (s. Abb.2), können Beschwerden sowohl in der Halswirbelsäule (HWS) mit zusätzlicher ausstrahlender Armproblematik als auch in der Lendenwirbelsäule (LWS) hervorrufen und für den Betroffenen eine erhebliche Verminderung der Lebensqualität bis hin zur Berufsunfähigkeit verursachen.

Der Arbeitsplatz des Ophthalmologen besteht aus Schreibtisch, Spaltlampe, Phoropter und Stuhl bzw. Hocker. Die Anordnung ist in Abb. 4 und 5 dargestellt. Bisher ist diese 
Ausstattung eher zweckorientiert als ergonomisch ausgerichtet. Beim Arbeiten mit der Spaltlampe (s. Abb.1) und auch beim langen Sitzen am OP-Mikroskop (s. Abb.2) werden besonders drei Bereiche der Wirbelsäule beansprucht. Zum einem wird der Kopf überstreckt und dadurch die HWS nach dorsal abgeknickt und ventral überstreckt. Somit kommt es zu einer ungleichmäßigen Belastung der Bandscheiben, welche eine frühzeitige Degeneration mit Fissurbildung im Anulus fibrosus der Bandscheiben zur Folge haben kann. Die vorgebeugte Haltung führt zu einer Verstärkung der konvexen Krümmung in der Brustwirbelsäule (BWS), also einer stärkeren Ausbildung der Kyphose. Im Bereich der LWS kommt es entgegen der anatomisch vorgegebenen Lordose zu einer stetigen Kyphosierung und somit auch hier zu einer stärkeren Belastung der Bandscheiben. Die so entstehenden unkontrollierten Fehlbewegungen zwischen den Wirbelsegmenten führen zu einer Kompression der Spinalnerven mit Schmerzsymptomatik und können zur Entstehung einer Protrusio bis hin zum Prolaps des Diskus führen. Die Arbeit am Phoropter (s. Abb. 4) zur Bestimmung der Sehschärfe und auch die indirekte Ophthalmoskopie (s. Abb. 3) belasten einseitig und unphysiologisch Schultergelenk und Schultergürtel, sowie den Nackenbereich. Aufgrund dieser Überbeanspruchung des Schultergelenks kann es langfristig zu einer Arthrose kommen. Durch die meist nicht vollständig ausgeführten Drehbewegungen zwischen Spaltlampe und Schreibtisch bei der Untersuchung, kommt es zu einer unphysiologischen und stark belastenden Verdrehung der Wirbelsäule, besonders in den Bereichen der HWS und der LWS. Dieses kann zur Entstehung eines Prolapses erheblich beitragen.

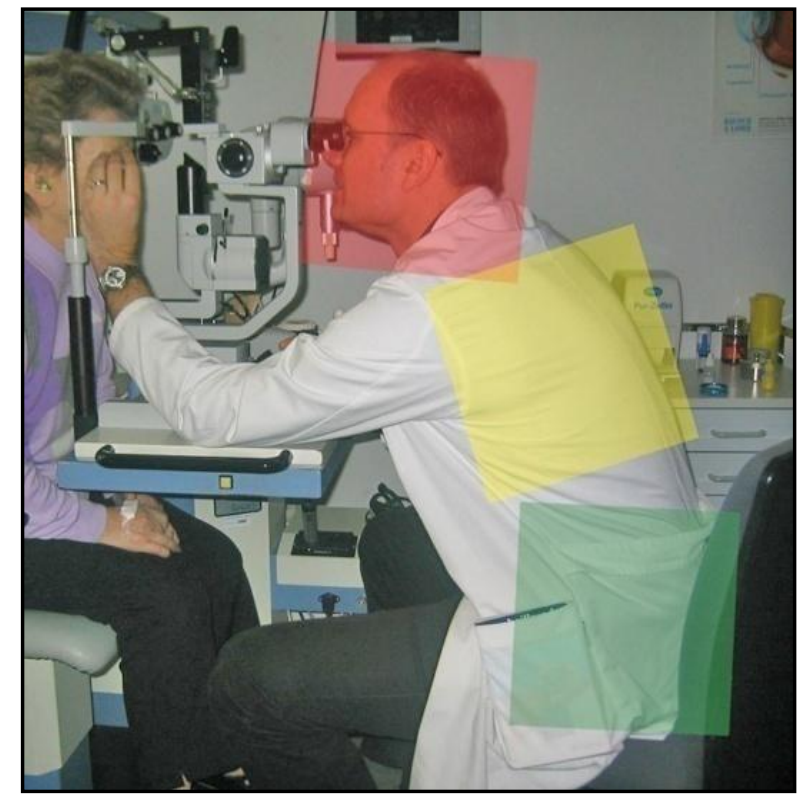

Abbildung 1: Sitzposition an der Spaltlampe

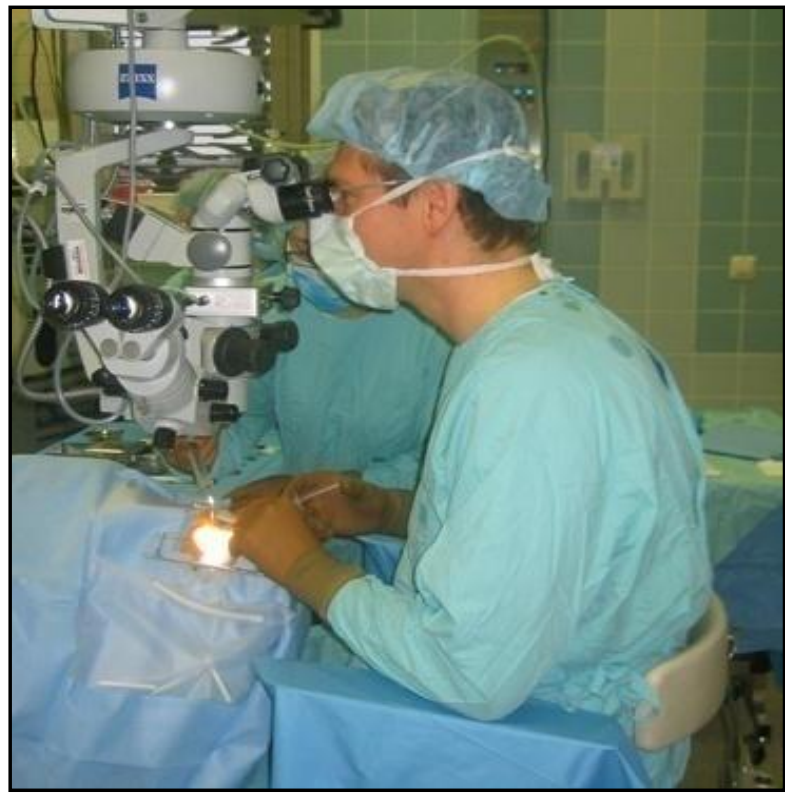

Abbildung 2: Sitzposition bei der Operation am Auge 


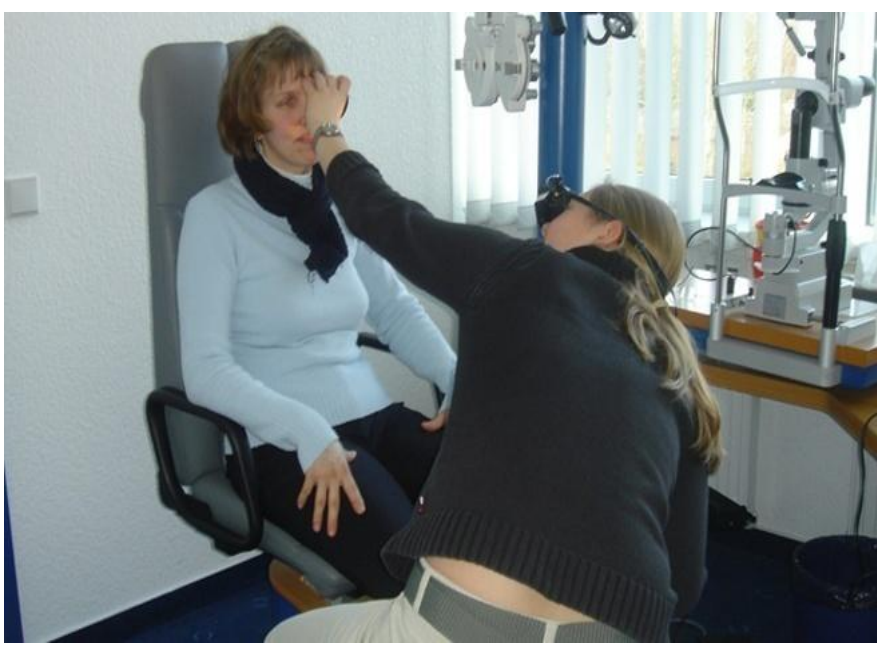

Abbildung 3: Indirekte Ophthalmoskopie

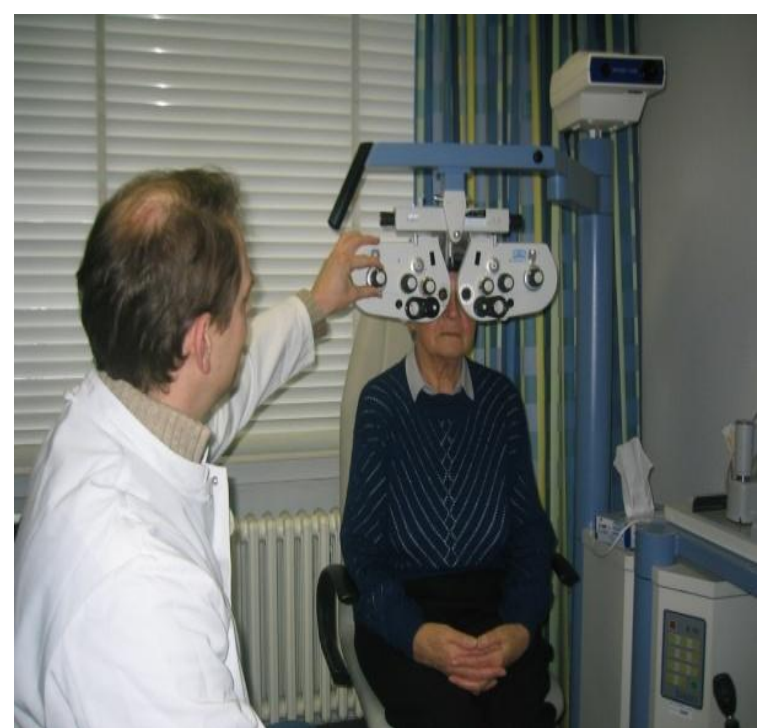

Abbildung 4: Sitzposition am Phoropter

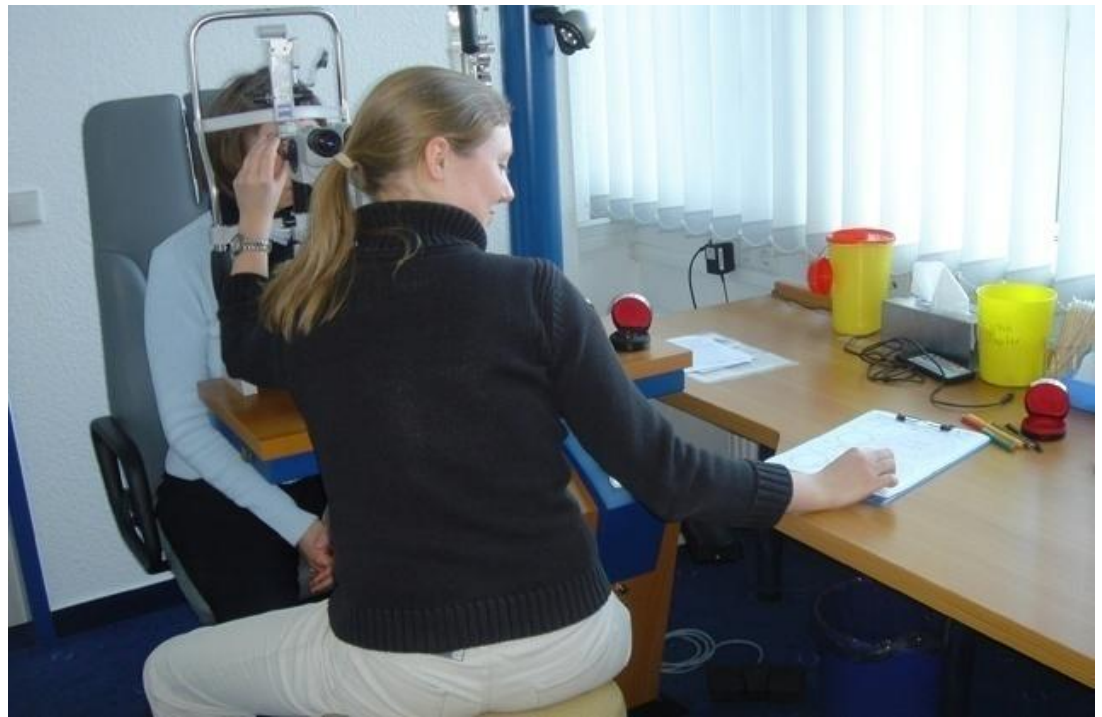

Abbildung 5: Drehbewegung zwischen Spaltlampe und Schreibtisch

(Quelle: Abb.1,2,4, Prof. Dr. med. H. Hoerauf, Augenklinik der Universität Göttingen; Abb. 3,5, PD Dr. med. S. Bopp, Augen-Tagesklinik Bremen) 


\subsection{Aktuelle Datenlage}

Ungefähr $90 \%$ der Menschen weltweit litten mindestens einmal in ihrem Leben an Rückenschmerzen. In der Allgemeinbevölkerung sind im Jahr $40-55 \%$ betroffen. (Carey 1989; Frank 1993) Aber nicht nur in der Allgemeinbevölkerung treten Rückenschmerzen vermehrt auf. Schon seit den 80er-Jahren belegen Studien ein häufigeres Auftreten von Rückenschmerzen unter Zahnärzten (Al Wazzan et al. 2001).

Es gibt vereinzelte Untersuchungen, die sich damit beschäftigt haben, dass Augenärzte in Bezug auf Rückenschmerzen möglicherweise einem größeren Risiko ausgesetzt sind als die Allgemeinbevölkerung, da diese lange in unphysiologischen Positionen arbeiten. In diesen Studien wurden verschiedene Aspekte in Bezug auf die augenärztliche Tätigkeit untersucht, wie zum Beispiel die Krafteinwirkung auf die Wirbelsäule durch die augenärztliche Tätigkeit. Des Weiteren wurde die Anzahl der Arbeitsjahre zu der Häufigkeit des Auftretens von Rückenschmerzen in Bezug gesetzt und in einer weiteren Studie betrachtete man verschiedene Bereiche des muskuloskeletalen Bewegungsapparates korreliert mit dem Stressniveau, dem Geschlecht, Patientenkontakt pro Woche, Arbeitsjahren und den verschiedenen Spezialisierungsrichtungen des Augenarztes. Eine Untersuchung dazu, inwiefern die Beschwerden mit dem Alter der Betroffenen und deren Tätigkeitsschwerpunkten im „konservativen“ und/oder „operativen“ Bereich korrelieren, liegt bisher nicht vor und soll daher mit der vorliegenden Studie erbracht werden.

In einer Studie des Zentrums für Physikalische und Rehabilitative Medizin des Sophien- und Hufeland - Klinikums Weimar zur Belastung der Wirbelsäule bei der augenärztlichen Tätigkeit (Seidel et al. 2005) wurde der Belastungswert (der Kraftwert, angegeben in Newton=N), der durch eine eingenommene Haltung auf die Wirbelsäule wirkt ermittelt. Dabei sind drei Arbeitshaltungen des Augenarztes untersucht worden: die Kopfophthalmoskopie, die direkte Ophthalmoskopie und die Untersuchung mit der Spaltlampe. (s. Abb.1 und 3) Die Studie ergab, dass der größte Belastungswert bei der Arbeit an der Spaltlampe entsteht. Hier wurde ein Wert von $1500 \mathrm{~N}$ gemessen, der auf den Bereich der Lenden- und Sakralwirbelsäule (L5/S1) wirkt. Damit wird konstitutionsabhängig (Größe, Gewicht, Konstitutionstyp und Gewebetyp) eine um 150\%ige höhere Belastung der lumbosakralen Übergangsregion durch die Spaltlampenuntersuchung provoziert, als in der Normalbevölkerung. Die Belastungswerte resultieren im Wesentlichen aus dem Vorneigewinkel und der Distanz der Arme zum Körper.

Eine englische Arbeit, die 1994 erhoben wurde, befasste sich mit Rückenschmerzen bei Augenärzten (Chatterjee et al. 1994). Es wurde eine Befragung per Fragebogen über die 
Arbeitsweise und das Auftreten von Rückenschmerzen bei allen Fachärzten der Augenheilkunde in England durchgeführt. Ein statistisch erhöhtes Risiko im Vergleich zur Allgemeinbevölkerung konnte nicht nachgewiesen werden.

Von den 325 Augenärzten, die an der Befragung teilgenommen haben, gaben 54\% ( $n=174)$ an, unter Rückenbeschwerden zu leiden. In der Studie wurde die Anzahl der Arbeitsjahre zu der Häufigkeit des Auftretens von Rückenschmerzen in Bezug gesetzt und es zeigte sich eine Zunahme der Beschwerden mit steigender Anzahl an Arbeitsjahren (>15 Arbeitsjahre). Des Weiteren wurden nach den Behandlungen gefragt, die durchgeführt wurden, um die Beschwerden zu lindern. 56\% $(n=97)$ der Teilnehmer gaben an, hauptsächlich Non Steroidal Anti - Inflammatory Drug (NSAIR) einzunehmen, 23\% $(n=40)$ der Teilnehmer gaben eine Physiotherapie an, $8 \%(n=14)$ der Teilnehmer haben alternative Behandlungen durchgeführt und $32 \%(n=56)$ machten keine Angaben.

Eine ähnliche 2005 in Nordamerika durchgeführte Umfrage (Dhimitri et al. 2005) erfasste muskuloskeletale Beschwerden im Bereich des Nackens, des unteren Rückens und der oberen Extremitäten. Diese drei Bereiche wurden korreliert mit dem Stressniveau, dem Geschlecht, Patientenkontakt pro Woche, Arbeitsjahren und den verschiedenen Spezialisierungsrichtungen des Augenarztes. Ergebnisse dieser Untersuchung zeigten, dass es bei einem hohen Stressniveau zu häufigeren Beschwerden im Nacken und im Bereich der oberen Extremitäten, sowie im Rückenbereich kommt. Hat ein Augenarzt beispielsweise mehr als 100 Patientenkontakte pro Woche, gab es eine positive Assoziation zu den Beschwerden im Nackenbereich. Augenärztinnen, die auf diese Studie geantwortet haben, klagten häufiger über Schmerzen im Bereich des Nackens und der oberen Extremitäten als ihre männlichen Kollegen, obwohl sie weniger Patientenkontakte und Behandlungen pro Woche durchführten.

Für die einzelnen Spezialisierungen in der Augenheilkunde ergab sich folgende Verteilung bezogen auf die Lokalisationen von Beschwerden: in der Allgemeinen Augenheilkunde waren die Beschwerden am häufigsten an den oberen Extremitäten $(66,3 \%)$, gefolgt von den Beschwerden im Nackenbereich (61,3\%) und den Beschwerden in der LWS (59,5\%). Bei den auf Netzhaut- und Glaskörperchirurgie spezialisierten Augenärzte standen die Beschwerden im Bereich der LWS (23,3\%) im Vordergrund, gefolgt von den Beschwerden im Nackenbereich (21,5\%) und den Beschwerden in den oberen Extremitäten (17,9\%). Bei den Kollegen mit Spezialisierungen auf Glaukom - Erkrankung, Plastische Augenheilkunde und Neuroophthalmologie gab es in den drei Beschwerdebereichen sehr niedrige Ergebniswerte.

Einschränkend muss erwähnt werden, dass nur 28\% ( $n=697)$ der Teilnehmer geantwortet haben. 


\subsection{Pilot - Fragebogen und Auswertung}

Aufgrund eigener Erfahrungen und entsprechender Berichte aus dem Kollegenkreis der Augenärzte/innen, haben im Jahre 2006 Frau PD Dr. med. S. Bopp aus Bremen und Professor Dr. med. H. Hoerauf aus Göttingen (beide operativ tätige Augenärzte) zusammen mit Frau L. - L. Sagebiel aus Lübeck (Physiotherapeutin) erstmals einen Kurs zur „richtigen Haltung als Augenarzt" auf dem größten deutschen Fortbildungskongress für Augenärzte, der Augenärztlichen Akademie Deutschland (AAD), angeboten. Die Nachfrage ist seither extrem hoch, so dass der Kurs seitdem jährlich ausgebucht ist. Dies unterstreicht die Bedeutung dieses Themas unter Augenärzten. Im Rahmen dieses Kurses wurde den Teilnehmern ein Fragebogen ausgehändigt. Mit diesem Fragebogen, der zehn Fragen umfasste, wollten die Verfasser einen ersten differenzierteren Eindruck über die Verbreitung muskuloskeletaler Beschwerden bei Augenärzten erhalten. Die Erfahrungen dieser Pilotstudie sollten als Grundlage dienen für seine Einsetzbarkeit zur Evaluation von Auswirkungen der augenärztlichen Tätigkeit auf die Wirbelsäule und dienten der Vorbereitung einer deutschlandweiten Umfrage unter Augenärzten.

Der Fragebogen wurde im selben Jahr 2006 nach statistischer Beratung durch Fr. Dr. med. C. Weiß (Koordinierungszentrum für klinische Studien Köln) auf sechzehn Fragen erweitert und genauer spezifiziert (siehe Anhang). Hierbei wurde insbesondere auf die Differenzierung des Geschlechtes, der Beschwerdedauer, der Leitsymptome, der Beschwerden an der Wirbelsäule bezogen auf die Region, die Extremitäten bzw. den Schulterbereich eingegangen. Mit diesem verbesserten Fragebogen wurde noch im gleichen Jahr eine neue Umfrage unter den Teilnehmern der Tagung der Retinologischen Gesellschaft in Kiel durchgeführt. Insgesamt haben sich 93 Augenärzte an dieser Befragung beteiligt, die sowohl aus dem Bereich der konservativen als auch der operativen Augenheilkunde stammten. Durch die Fachausrichtung des Kongresses war allerdings eine gewisse Selektion von Augenärzten mit dem Spezialgebiet Netzhauterkrankungen gegeben. 
Die Auswertung des Pilot - Fragebogen ergab folgendes Ergebnis: 34 der 93 befragten Teilnehmer waren männlich (37\%), 56 Teilnehmer weiblich (60\%). 3 Teilnehmer (3\%) machten diesbezüglich keine Angaben. (s. Abb.6)

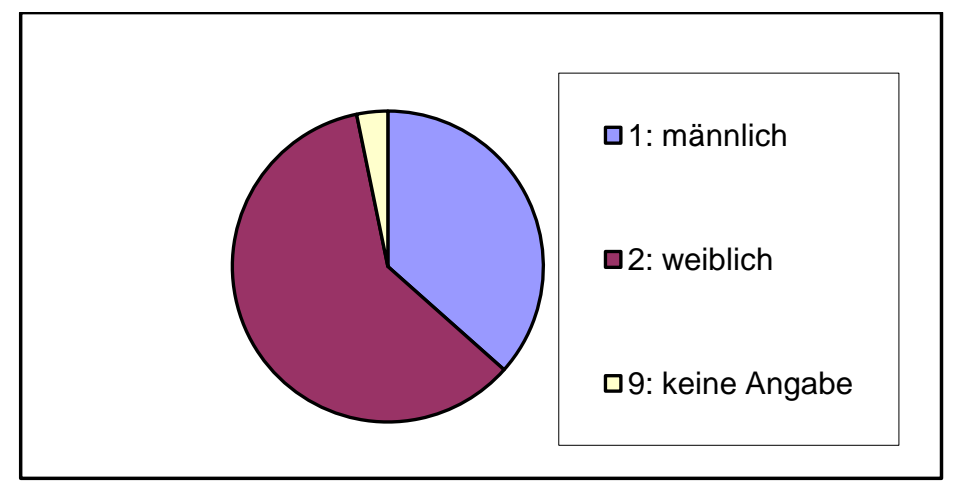

Abbildung 6: Gechlechterverteilung der Augenärzte/innen innerhalb der Umfrage unter den Teilnehmern der Tagung der Retinologischen Gesellschaft in Kiel 2006 (Weiß 2006/2008)

In der Frage, in der nach der Dauer der Beschwerden gefragt wurde, ist gleichzeitig auch nach der Art der Beschwerden gefragt worden. 10 Teilnehmer (11\%) gaben an, akute Beschwerden zu haben, 31 Teilnehmer (33\%) klagten über chronische Beschwerden und 52 Teilnehmer (56\%) machten hier keine Angaben. (s. Abb. 7)

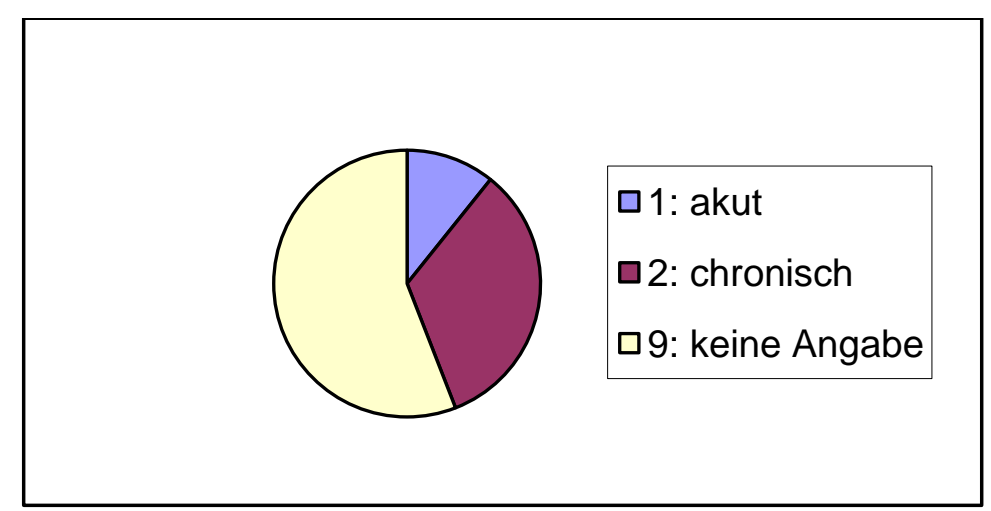

Abbildung 7: Beschwerdeart klassifiziert nach akut, chronisch und keine Angabe innerhalb der Umfrage unter den Teilnehmern der Tagung der Retinologischen Gesellschaft in Kiel 2006 (Weiß 2006/2008) 
Die Dauer der Beschwerden bezog sich auf die Wirbelsäulenbeschwerden. Dabei bestanden diese bei 3 Teilnehmern (3\%) seit kurzem, bei 4 Teilnehmern (4\%) weniger als ein Jahr, bei 43 Teilnehmern (46\%) schon länger als ein Jahr und 43 Teilnehmer (46\%) machten hierzu keine Angaben. (s.Abb.8)

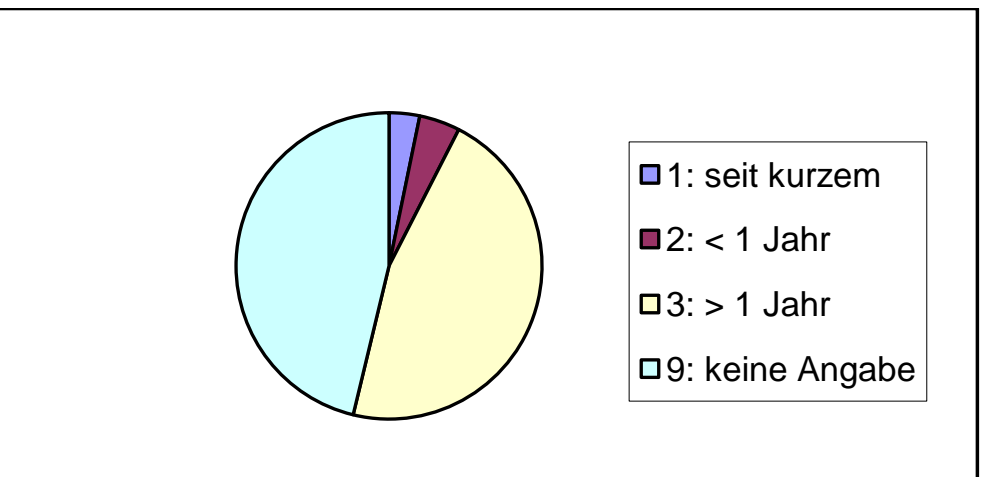

Abbildung 8: Beschwerdedauer (seit kurzem, <1Jahr, >1Jahr, keine Angaben) innerhalb der Umfrage unter den Teilnehmern der Tagung der Retinologischen Gesellschaft in Kiel 2006 (Weiß 2006/2008)

Bei den Teilnehmern zeigte sich eine Lokalisationshäufigkeit der Beschwerden in den einzelnen Bereichen mit unterschiedlicher Gewichtung. Besonders stark war der Bereich der LWS mit $65 \%$ betroffen, gefolgt von den Beschwerden in der HWS mit $50 \%$. Die Häufigkeit der Beschwerden an der Schulter war mit 20\% höher als die Häufigkeit der Beschwerden an der BWS mit 17\%, sowie den an den oberen Extremitäten mit $7 \%$ und den unteren Extremitäten mit 5\%. (s. Abb. 9)

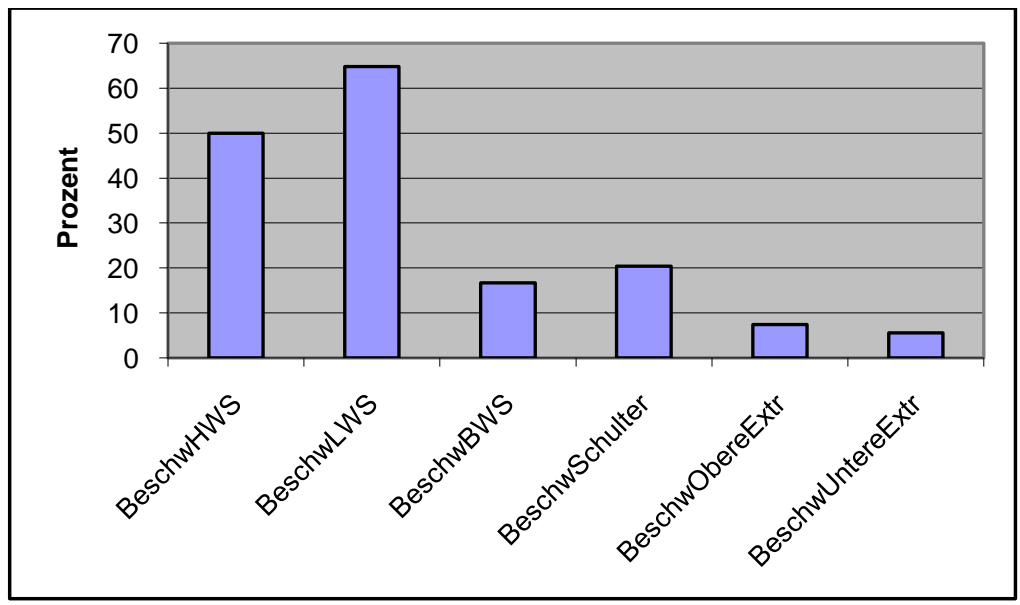

Abbildung 9: Häufigkeiten der Beschwerdelokalisation (in \%) (Beschwerden an der Halswirbelsäule (HWS), Beschwerden an der Lendenwirbelsäule (LWS), Beschwerden an der Brustwirbelsäule (BWS), Beschwerden an der Schulter, Beschwerden an der oberen Extremität, Beschwerden an der unteren Extremität) innerhalb der Umfrage unter den Teilnehmern der Tagung der Retinologischen Gesellschaft in Kiel 2006 (Weiß 2006/2008) 
Bei der Frage zu den Leitsymptomen der muskuloskeletalen Beschwerden gaben 72\% Schmerzen, 35\% Immobilität und 29\% ein Steifheitsgefühl an. 22\% der Teilnehmer klagten über Parästhesien und ungefähr 2\% äußerten andere Leitsymptome. (s. Abb. 10)

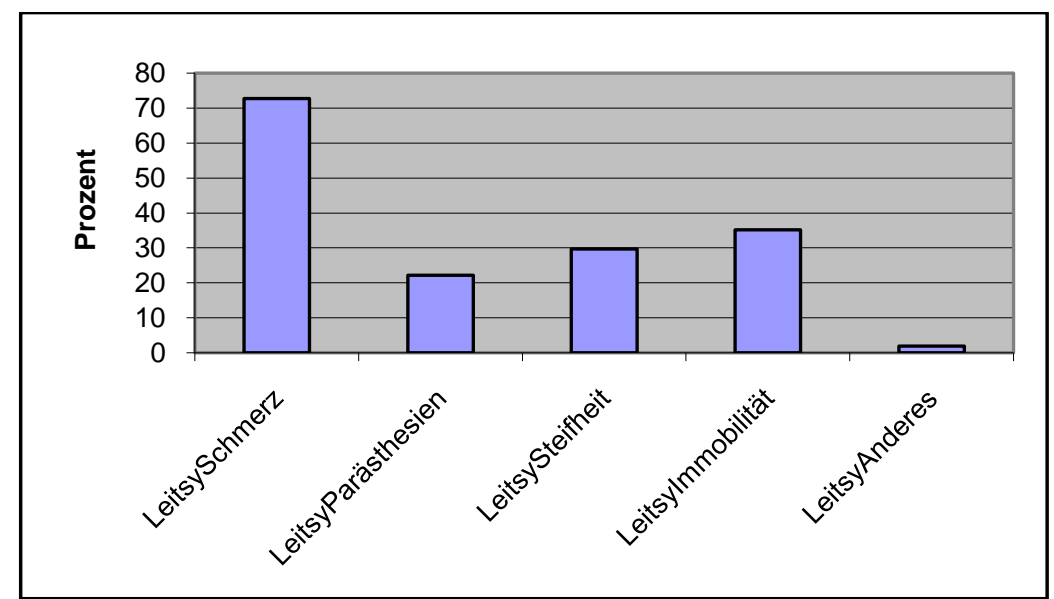

Abbildung 10: Häufigkeiten der Leitsymptome (in \%) (Schmerzen, Parästhesien, Steifheit, Immobilität, andere Leitsymptome) innerhalb der Umfrage unter den Teilnehmern der Tagung der Retinologischen Gesellschaft in Kiel 2006 (Weiß 2006/2008) 


\subsection{Zielsetzung und Fragestellung}

Die Erfahrungen aus den dargestellten selektiven Befragungen von Augenärzten zeigen, dass ein erheblicher Anteil von ihnen an muskuloskeletalen Beschwerden leidet. Ob dieser Eindruck repräsentativ für alle Augenärzte ist und ob bestimmte fachspezifische Tätigkeiten die Entwicklung dieser Beschwerden begünstigen, ist unklar.

Ziel der vorliegenden Untersuchung war daher die Durchführung einer Befragung an einer repräsentativen Gruppe von Augenärzten unter besonderer Berücksichtigung fachspezifischer ergonomischer Belastungen, um Aussagen über Häufigkeit der Beschwerden, Vorschäden und mögliche Einflussfaktoren beruflicher und außerberuflicher Art treffen zu können, insbesondere im Vergleich zur Häufigkeit in der Allgemeinbevölkerung. Die Ergebnisse können zur Ableitung spezifischer Präventionsmaßnahmen in der Augenheilkunde beitragen.

Auf der Basis eines Fragebogens wurden daher als Zielparameter definiert:

- Augenärzte/innen mit „Vorschäden“ (Frage 5)

- Augenärzte/innen mit „aktuelle Beschwerden“ (Frage 6)

Des Weiteren wird geschaut, ob die Häufigkeitsverteilung von muskuloskeletalen Beschwerden bei Augenärzten von Einflussfaktoren bestimmt wird.

Zu diesen Einflussfaktoren zählen:

- biographische Faktoren: Alter in Jahren (Frage 1); Geschlecht (Frage 1) und Berufsjahre (Frage 3a)

- berufsspezifische Faktoren: augenärztliche Spezialisierungen (Frage 2); die Tätigkeitsschwerpunkte „konservativ“ und/oder „operativ“ (Frage 3a) und spezielle Operationen (Frage 4)

- private Faktoren: Sport (Frage 8).

Die Zielfaktoren und Einflussfaktoren wurden jeweils in den Ausprägungen (ja/nein) bei den Fragen $2 ; 5 ; 6 ; 8$ und 9 sowie $(\mathrm{m} / \mathrm{w})$ bei der Frage 1 erfasst. 


\section{Material und Methoden}

\subsection{Fragebogen - Erstellung}

Ausgehend vom Pilot - Fragebogen für die Tagung der Retinologischen Gesellschaft im Jahre 2006, wurde in Zusammenarbeit mit Frau PD Dr. A. Heutelbeck und Herrn Prof. E. Hallier, beide aus der Abteilung Arbeitsmedizin und Sozialmedizin der Universitätsmedizin Göttingen (UMG), sowie Herrn Prof. R. Hilgers aus der Abteilung Medizinische Statistik der UMG ein neuer Fragebogen erstellt.

\subsection{Erstellung des BVA - Umfragebogens}

\subsubsection{Konsequenzen aus dem Pilot - Fragebogen und Änderungen}

Im einzelnem wurden folgende Fragen des Pilot - Fragebogens (siehe Anhang) verändert:

Frage eins und Frage zwei wurden miteinander verbunden, so dass Alter und Geschlecht nun als Antwort auf eine Frage anzugeben waren.

Auch Frage drei „Welchen Arbeitsschwerpunkt in der Augenheilkunde haben Sie?" und Frage vier „Wie lange arbeiten Sie bereits im augenärztlichen Fachgebiet?“ wurden zu einer Frage zusammengefasst. Somit konnten zwei Gruppierungen erstellt werden, um eine Auswertung der Daten zu ermöglichen.

Die Frage fünf „Wie/Wo sind Sie beschäftigt?“ erschien als nicht relevant, da die Art der Beschäftigung (selbständig/angestellt) und der Ort der Beschäftigung (Praxis/Krankenhaus) in Bezug auf die Beschwerden an der Wirbelsäule und den Extremitäten keinen genauen Zusammenhäng erkennen ließ.

Frage sechs „Sind bei Ihnen morphologische Schäden an der Wirbelsäule bekannt?" ist ungenau und wurde präzisiert nach Vorschäden an der Wirbelsäule und den Extremitäten.

Frage neun „Wo haben Sie am meisten Beschwerden?" wurde weiter differenziert in muskuläre und neurologische Beschwerden der Extremitäten und als Unterfrage bei Frage sechs aufgeführt.

Frage zehn „Haben Sie wegen Ihrer Beschwerden eine Behandlung durchgeführt?" und Frage elf „Welche Konsequenzen haben Sie angesichts Ihrer Beschwerden ergriffen?" konnten zu einer Frage zusammengefasst werden und bildeten eine weitere Unterfrage der Frage sechs.

Die Frage dreizehn „Wie viele Patienten sehen Sie pro Woche?“ erschien ebenfalls wenig sinnvoll, da aufgrund ungenauer Angaben kein kausaler Zusammenhang zwischen der 
Dauer der Tätigkeit des Augenarztes und der Anzahl Patientenkontakte hergestellt werden konnte.

\subsubsection{Gliederung und Fragenkomplexe}

Bei der Gliederung der Fragen zur Zusammenstellung des neuen Fragebogens für die große BVA - Umfrage erschien es sinnvoll, zuerst nach den persönlichen Daten, der beruflichen Tätigkeit als Augenarzt in Bezug auf das Spezialgebiet, den Arbeitsbereich „konservativ“ und/oder "operativ“ und die Arbeitsjahre und Arbeitsstunden pro Woche, sowie nach der Anzahl operativer Eingriffe zu fragen. (Frage 1-4)

Frage fünf nach den Vorschäden und anderen Erkrankungen an der Wirbelsäule sollte der Einleitung beziehungsweise Überleitung zum eigentlichen Thema, den muskuloskeletalen Beschwerden unter Augenärzten, dienen.

Der nächste Fragenkomplex sollte die aktuellen Beschwerden an der Wirbelsäule und den Extremitäten mit Bezug auf die Symptome, die häufigste Lokalisation der Beschwerden, die Tätigkeit mit den stärksten Beschwerden und die selbst eingeleiteten Behandlungen erfassen.

Abschließend wird das Gesamtausmaß der Beschwerden abgefragt sowie bereits ausgeübte Präventionsmaßnahmen durch Physiotherapie, Gymnastik, Sport und ergonomische Gestaltung des Arbeitsplatzes.

\subsubsection{Probelauf}

Im Februar 2009 wurde ein Probelauf dieses optimierten Fragebogens bei niedergelassenen Augenärzten/innen aus Augenarztpraxen in Göttingen und bei angestellten Kolleginnen/-gen in der Augenklinik der UMG durchgeführt. Insgesamt wurden zehn Fragebogen persönlich verteilt, wovon acht zurück gesendet wurden. Dieser Probelauf mit niedriger Fallzahl sollte mögliche Unklarheiten in der Formulierung der Fragestellungen und Probleme beim Ausfüllen des Bogens erfassen.

Aufgrund dieses Testes gab es nochmals fünf wesentliche Veränderungen am Fragebogen: Frage drei „Führen Sie Laserbehandlungen durch?“ und Frage zehn „Haben Sie schon mal an den Kursen ,Die richtige Haltung als Augenarzt' auf den AAD -Tagungen teilgenommen?“ wurden entfernt. Ferner resultierte eine Umstrukturierung bei den Unterfragen von Frage sechs, wobei Frage sechs c "Wo haben Sie die Beschwerden“ entfernt wurde. Frage acht nach der Sportart wurde aufgrund der umfangreichen verschiedenen Sportarten entfernt. Frage neun „Könnten Sie sich vorstellen, dass durch eine Optimierung Ihres Arbeitsplatzes eine Linderung der Beschwerden zu erzielen wäre?“ wurde erweitert und verbessert. 


\subsection{Logistische Vorbereitung der BVA - Umfrage}

Die Durchführung der Befragung von muskuloskeletalen Beschwerden unter Augenärzten in Deutschland wurde in Zusammenarbeit mit dem BVA durchgeführt, da fast alle Augenärzte/innen Mitglieder des BVA sind. Der BVA übernahm dabei die Versendung des Fragebogens und des Begleitschreibens per Post. Die Portokosten für die Versendung übernahm im Rahmen eines Sponsoring - Vertrages die Firma bon Optik aus Lübeck.

Insgesamt wurden 5954 Mitglieder des BVA angeschrieben.

Für die Rücksendung des Fragebogens gab es drei Möglichkeiten:

a) Die Teilnehmer konnten den Fragebogen per Post an die angegebene Adresse zurücksenden.

b) Die Teilnehmer konnten den Bogen per Fax an eine Nummer zurücksenden.

c) Die Teilnehmer konnten den Fragebogen via Internet ausfüllen und absenden.

Für die Option c) wurde in Zusammenarbeit mit der Abteilung „Informationstechnologie Applikationen Forschung und Lehre" der UMG eine Onlineversion des Fragebogens angefertigt und mit Hilfe des Programms EvaSys online gestellt. Für den Online-Zugang gab es ein gemeinsames Passwort, welches den befragten Augenärzten im Anschreiben mitgeteilt wurde. 


\subsection{Erfassung und Auswertung des BVA - Fragebogens}

Zur Auswertung und zur Erfassung des schriftlichen Fragebogens und der Onlineversion des Fragebogens halfen verschiedene Computerprogramme. Das Programm Access 2007 von Microsoft Office diente zur Erfassung und Codierung des Fragebogens, der mit der Post oder per Fax zurück kam. Zur Bearbeitung wurde eine Fragebogen - Maske, welche dem Original - Fragebogen entsprach, mit dem gleichen Programm erstellt. Somit konnten Eingabegrenzen für die Erfassung der Daten erstellt und die Fehlereingabe minimiert werden. Gleichzeitig erhielten die Fragenbögen eine Nummerierung, die dann per Hand auf die einzelnen Fragebögen aufgetragen wurde.

Die Onlinedaten des Fragebogens und die Erfassung der zurück geschickten Bögen wurden später zusammengefügt, wobei sie die gleiche Codierung hatten. Die Auswertung und Erstellung von Graphen wurde mit Excel 2007 Microsoft Office und dem Statistica Version 8 Programm (Firma StatSoft) durchgeführt.

Für die weitere Auswertung der Daten war es hilfreich, diese in Gruppen einzuteilen. Zuerst wurden die Augenärzte/innen nach ihren gesamt geleisteten Berufsjahren ( $<10$ Berufsjahre, 10-20 Berufsjahre, 20-30 Berufsjahre, 30-45 Berufsjahre) und anschließend nach den Tätigkeitsschwerpunkten konservativ, operativ und der Kombination von konservativ und operativ gruppiert. Diese konnten dann in Bezug auf die Fragestellungen miteinander verglichen werden. (s. Abb. 11)

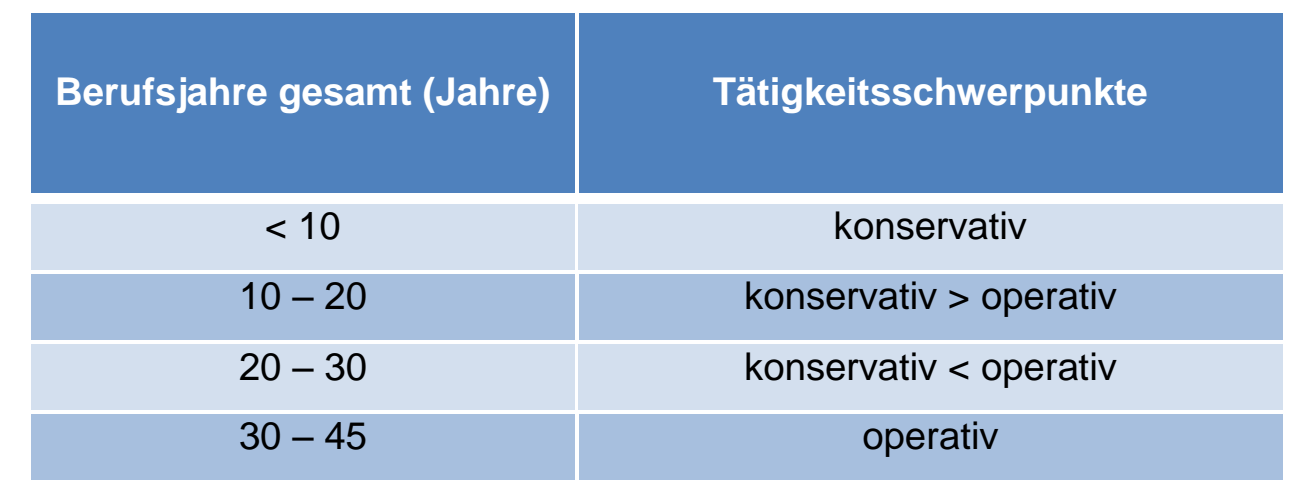

Abbildung 11: Darstellung der Gruppen Einteilung Berufsjahre gesamt in Jahre und der Tätigkeitsschwerpunkte konservativ, operativ und der Kombination von konservativ und operativ 


\section{Ergebnisse}

\subsection{Fragebogen - Rücklaufquote}

Im Frühjahr 2009 wurden 5954 Fragebögen an die Mitglieder des Berufsverbandes der Augenärzte versandt. Insgesamt haben 1861 (31\%) der Augenärzte/innen geantwortet. Von diesen 1861 Fragebögen konnten 54 Fragebögen nicht gewertet werden, da bei der Rückübersendung per Fax Fehler auftraten, zum einen kamen von dem zweiseitigen Fragebogen nur eine Seite an oder nur leere Blätter. Insgesamt kamen somit 1807 (30\%) der verschickten Frageböge zur Auswertung. Einige der Teilnehmer machten keine Angaben (missing values), diese sind in der untenstehenden Tabelle aufgeführt. (s. Abb. 12)

\begin{tabular}{|c|c|c|c|}
\hline Frage & $\begin{array}{l}\text { Rückmeldungen } \\
\text { gesamt }\end{array}$ & $\begin{array}{l}\text { korrekte } \\
\text { Rückmeldungen }\end{array}$ & $\begin{array}{l}\text { keine Angaben } \\
\text { (missing values) }\end{array}$ \\
\hline 1. Alter & 1807 & 1800 & 7 \\
\hline 1. Geschlecht & 1807 & 1789 & 18 \\
\hline 2. Spezialgebiet & 1807 & 1751 & 56 \\
\hline 3. Tätigkeitsschwerpunkte & 1807 & 1744 & 63 \\
\hline 3. Berufsjahre gesamt & 1807 & 1755 & 52 \\
\hline 5. Vorschäden & 1807 & 1800 & 7 \\
\hline 6. aktuelle Beschwerden & 1807 & 1803 & 4 \\
\hline 8. Sport & 1807 & 1796 & 11 \\
\hline 9. Optimierung des & & & \\
\hline Arbeitsplatzes & 1807 & 1596 & 211 \\
\hline
\end{tabular}

Abbildung 12: Darstellung des Gesamtkollektivs der zur Auswertung stehenden Augenärzte/innen und deren korrekte Rückmeldungen zu den einzelnen Fragen und die Auflistung fehlender Antworten (missing values) 


\subsection{Kollektivbeschreibung}

Eingeschlossen wurden bei den 1807 Augenärzten, 51\% Augenärztinnen ( $n_{\text {gesamt }}=913$; Alter: $\min =28 ; \max =73 ; M W=49$; Median=49; $\mathrm{Q} 1=43 ; \mathrm{Q} 3=55)$ und 48\% Augenärzte $\left(\mathrm{n}_{\text {gesamt }}=876\right.$; Alter: $\min =27 ; \max =76 ; \mathrm{MW}=52 ;$ Median=51; $\mathrm{Q} 1=45 ; \mathrm{Q} 3=58$ ), mit einem mittleren Alter von 50 Jahren $(\min =27$ Jahre; $\max =76$ Jahre; Median=50; $Q 1=44 ; Q 3=57)$.

Hinsichtlich der verschiedenen Spezialgebiete der Augenheilkunde gaben 15\% $\left(n_{\text {gesamt }}=266\right.$; $\mathrm{n}_{\text {männlich }}=189 ; \mathrm{n}_{\text {weiblich }}=76 ; \mathrm{n}_{\mathrm{kAGeschlecht}}=1 ;$ Alter: $\min =32 ; \max =68 ; \mathrm{MW}=47 ;$ Median=46; $\mathrm{Q} 1=42$; Q3=52) der Teilnehmer das Spezialgebiet Vorderabschnitt an. 9\% $\left(n_{\text {gesamt }}=160 ; n_{\text {mänlich }}=78\right.$; $\mathrm{n}_{\text {weiblich }}=82$; Alter: $\min =34$; $\max =76 ; \mathrm{MW}=52 ;$ Median=51; $\mathrm{Q1}=45 ; \mathrm{Q} 3=57$ ) das Spezialgebiet Strabologie und nur 4\% $\left(n_{\text {gesamt }}=78 ; n_{\text {männlich }}=52 ; n_{\text {weiblich }}=25 ; n_{\text {kAGeschlecht }}=1 ;\right.$ Alter: min=33; $\max =67 ; \quad M W=49 ; \quad$ Median=48; $Q 1=43 ; \quad Q 3=55)$ der Teilnehmer das Spezialgebiet Hinterabschnitt. Die Mehrzahl der Teilnehmer 69\% $\left(\mathrm{n}_{\text {gesamt }}=1247 ; \mathrm{n}_{\text {männlich }}=532 ; \mathrm{n}_{\text {weiblich }}=701\right.$; $\mathrm{n}_{\mathrm{kAGeschlecht}}=14 ;$ Alter: $\min =27 ; \max =74 ; \mathrm{MW}=50 ;$ Median=51; $\mathrm{Q} 1=44 ; \mathrm{Q} 3=57$ ) hat kein Spezialgebiet in der Augenheilkunde, (missing values 3\%; $n=56$ ). (s. Abb. 13)

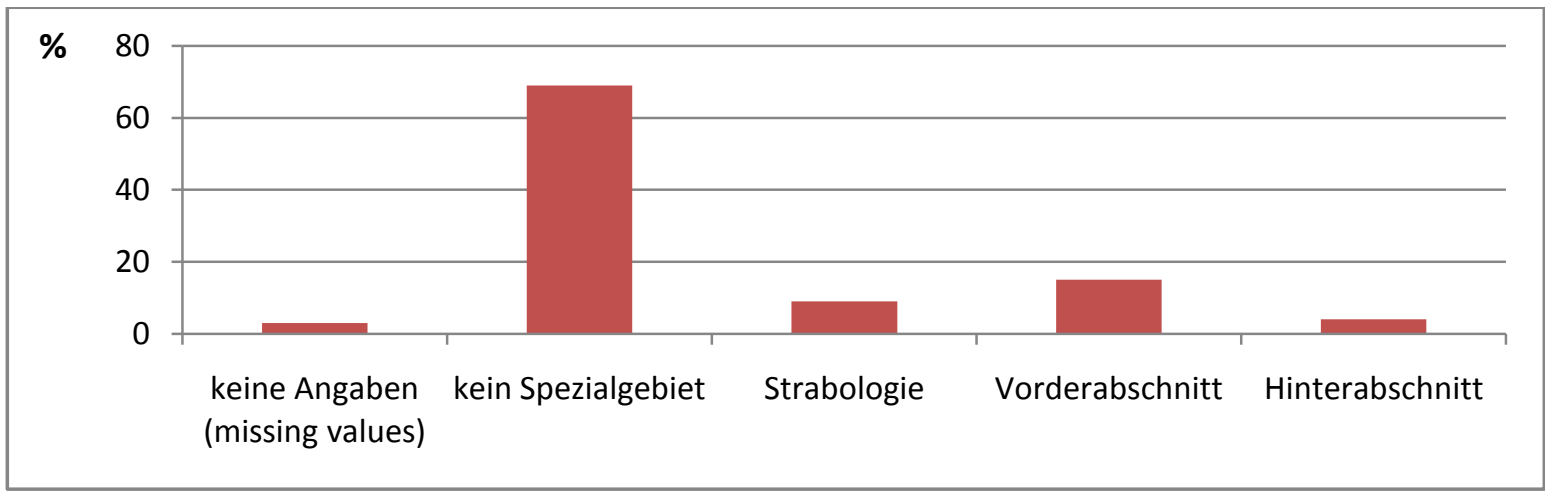

Abbildung 13: Verteilung des Gesamtkollektivs der Augenärzte/innen in den Spezialgebieten (in \%) Strabologie, Vorderabschnitt und Hinterabschnitt der Augenheilkunde und Auflistung der Augenärzte/innen, die kein Spezialgebiet haben, und der fehlenden Angaben zu dieser Frage (missing values)

Bei der Frage: „In welchem Arbeitsbereich der Augenheilkunde arbeiten Sie heute vorwiegend (h/Woche; konservativ oder operativ)?" $58 \%$ ( $n=1050)$ gaben an, überwiegend rein konservativ, 1\% $(n=26)$ rein operativ und 38\% $(n=689)$ gleichwertig operativ/konservativ tätig zu sein, (missing values $2 \% ; n=42$ ).

Hinsichtlich des konservativen Arbeitsbereichs besteht eine mittlere Wochenarbeitszeit von $35 \mathrm{~h} /$ Woche, $\left(\min 2 \mathrm{~h} /\right.$ Woche bis $\max 83 \mathrm{~h} /$ Woche; $\mathrm{n}_{\text {gesamt }}=1738 ; \mathrm{n}_{\text {mänlich }}=839 ; \mathrm{n}_{\text {weiblich }}=882$; $\mathrm{n}_{\text {kAGeschlecht }}=17$; Alter: $\min =27$; $\max =76 ; \mathrm{MW}=50 ;$ Median=50; $\mathrm{Q} 1=44 ; \mathrm{Q} 3=57$ ). Bei dem operativen Arbeitsbereich besteht eine mittlere Wochenarbeitszeit von 4h/Woche (min 
$1 \mathrm{~h} /$ Woche bis $\max 60 \mathrm{~h} /$ Woche; $\mathrm{n}_{\text {gesamt }}=715 ; \mathrm{n}_{\text {mänlich }}=458 ; \mathrm{n}_{\text {weiblich }}=254 ; \mathrm{n}_{\text {kAGeschlecht }}=3 ;$ Alter: $\min =29 ; \max =76 ; \mathrm{MW}=48 ;$ Median=47; $\mathrm{Q} 1=42 ; \mathrm{Q} 3=54)$.

Auf die Frage nach den bisher gesamt geleisteten Berufsjahren gaben die Teilnehmer $(n=1755)$ eine Dauer von 1 bis 45 Jahren an $\left(n_{\text {männlich }}=864 ; n_{\text {weiblich }}=877 ; n_{\text {kAGeschlecht }}=14 ;\right.$ Alter: $\min =27$; $\max =76 ; \mathrm{MW}=50$; Median=50; $\mathrm{Q} 1=44 ; \mathrm{Q} 3=57$ ) ( missing values $\mathrm{n}=52$ )).

Gruppiert man die Berufsjahre, waren 5\% ( $n_{\text {gesamt }}=97 ; n_{\text {mänlich }}=32 ; n_{\text {weiblich }}=64 ; n_{\text {kAGeschlecht }}=1$; Alter: $\min =27$; $\max =44 ; \mathrm{MW}=35$; Median=35; $\mathrm{Q1}=33 ; \mathrm{Q} 3=37$ ) der Teilnehmer in der Gruppe „<10 Berufsjahre“ tätig. Die Gruppe „10-20 Berufsjahre“ umfasste 32\% ( $\mathrm{n}_{\text {gesamt }}=578$; $\mathrm{n}_{\text {männlich }}=278 ; \mathrm{n}_{\text {weiblich}}=297 ; \mathrm{n}_{\mathrm{kAGeschlecht}}=3 ;$ Alter: $\min =35 ; \max =72 ; \mathrm{MW}=43 ;$ Median=43; $\mathrm{Q1}=40$; Q3=46), die Gruppe „20-30 Berufsjahre“, 37\% ( $n_{\text {gesamt }}=666 ; n_{\text {männlich }}=323 ; n_{\text {weiblich }}=337$; $\mathrm{n}_{\text {kAGeschlecht }}=6$; Alter: $\min =44$; $\max =65 ; \mathrm{MW}=52$; Median=51; $\mathrm{Q1=49;} \mathrm{Q} 3=54$ ) der Teilnehmer. In der Gruppe „30-45 Berufsjahre“, sind 23\% ( $\mathrm{n}_{\text {gesamt }}=414 ; \mathrm{n}_{\text {männlich }}=231 ; \mathrm{n}_{\text {weiblich }}=179$; $\mathrm{n}_{\mathrm{kAGeschlecht}}=4$; Alter: $\min =53$; $\max =76 ; \mathrm{MW}=61 ;$ Median=60; $\left.\mathrm{Q1}=58 ; \mathrm{Q} 3=64\right)$. Unter den 1807 machten $3 \%\left(n_{\text {gesamt }}=52 ; n_{\text {männlich }}=12 ; n_{\text {weiblich }}=36 ; n_{\text {kAGeschlecht }}=4 ;\right.$ Alter: $\min =33 ; \max =69$; $\mathrm{MW}=44$; Median=52; $\mathrm{Q1=40;} \mathrm{Q} 3=56$ ) keine Angaben zu Ihren bisher gesamten geleisteten Berufsjahren (missing values). (s. Abb. 14)

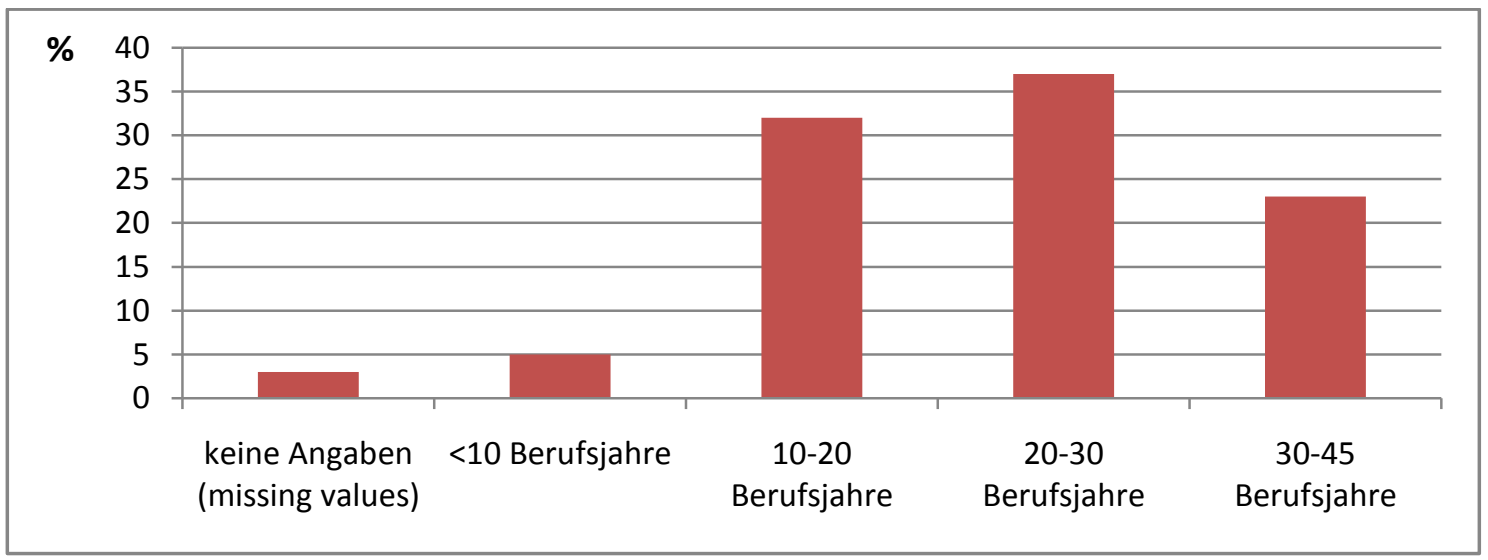

Abbildung 14: Verteilung des Gesamtkollektivs der Augenärzte/innen in den Gruppen der geleisteten Berufsjahre (in \%) (<10 Berufsjahre, 10-20 Berufsjahre, 20-30 Berufsjahre, 30-45 Berufsjahre) und die Angaben der fehlenden Aussagen (missing values) zu der Angabe der gesamt geleisteten Berufsjahre

Bezüglich der Frage nach den einzelnen Tätigkeitsschwerpunkten (konservativ und operativ) sind $31 \%\left(n_{\text {gesamt }}=569 ; n_{\text {männlich }}=174 ; n_{\text {weiblich }}=388 ; n_{\text {kAGeschlecht }}=7 ;\right.$ Alter: $\min =27 ; \max =73$; $\mathrm{MW}=50 ;$ Median=50; $\mathrm{Q1}=44 ; \mathrm{Q} 3=57$ ) der Teilnehmer rein konservativ tätig. $50 \%$ $\left(n_{\text {gesamt }}=900 ; n_{\text {männlich }}=486 ; n_{\text {weiblich }}=406 ; n_{\text {kAGeschlecht }}=8 ;\right.$ Alter: $\min =30 ; \quad \max =76 ; \quad M W=51$; Median=51; $Q 1=45 ; Q 3=58$ ) der Teilnehmer sind mehr konservativ als operativ tätig. 13\% $\left(\mathrm{n}_{\text {gesamt }}=232 ; \mathrm{n}_{\text {männlich }}=160 ; \mathrm{n}_{\text {weiblich }}=70 ; \mathrm{n}_{\text {kAGeschlecht }}=2 ;\right.$ Alter: $\min =33 ; \max =74 ; \quad M W=48$; 
Median=47; Q1=42; Q3=54), der Teilnehmer sind mehr operativ als konservativ tätig und 2\% $\left(n_{\text {gesamt }}=43 ; \quad n_{\text {männlich }}=26 ; \quad n_{\text {weiblich }}=16 ; \quad n_{\text {kAGeschlecht }}=1 ;\right.$ Alter: $\min =39 ; \quad \max =67 ; \quad M W=50$; Median=48; Q1=44; Q3=54) der Teilnehmer sind rein operativ tätig. Von den 1807 machten $3 \%\left(n_{\text {gesamt }}=63 ; n_{\text {männlich }}=30 ; n_{\text {weiblich }}=33 ;\right.$ Alter: $\min =34 ; \max =69 ; M W=51 ;$ Median=50; $Q 1=44$; $\mathrm{Q} 3=58$ ) keine Angaben (missing values) zu den einzelnen Tätigkeitsschwerpunkten konservativ und operativ.

Die operativ tätigen Augenärzte führten insgesamt 37\% $\left(n_{\text {gesamt }}=638\right)$ Laser Behandlungen, $21 \%\left(n_{\text {gesamt }}=373\right)$ Katarakt Operationen, 21\% $\left(n_{\text {gesamt }}=373\right)$ Lidoperationen, 9\% $\left(n_{\text {gesamt }}=154\right)$ Netzhaut Operationen und $16 \%\left(n_{\text {gesamt }}=271\right)$ sonstige Operationen durch.

Betrachtet man Wirbelsäulen-relevante Faktoren aus dem privaten Bereich, spielte der Faktor Sport unter den Teilnehmern wie folgt eine Rolle, 84\% $\left(n_{\text {gesamt }}=1514 ; n_{\text {männlich }}=739\right.$; $\mathrm{n}_{\text {weiblich }}=760 ; \mathrm{n}_{\text {kAGeschlecht }}=15$; Alter: $\min =27 ; \max =76 ; \mathrm{MW}=50 ;$ Median=50; $\mathrm{Q1}=44 ; \mathrm{Q} 3=56$ ) gaben an, aktiv Sport zu betreiben, $16 \% \quad\left(n_{\text {gesamt }}=282 ; \quad n_{\text {männlich }}=134 ; \quad n_{\text {weiblich }}=145\right.$; $\mathrm{n}_{\mathrm{kAGeschlecht}}=3$; Alter: $\min =31$; $\max =73 ; \mathrm{MW}=51$; Median=52; $\mathrm{Q} 1=45 ; \mathrm{Q} 3=58$ ) betreiben keinen Sport und $0,6 \% \quad\left(n_{\text {gesamt }}=11 ; n_{\text {männlich }}=3 ; n_{\text {weiblich }}=8 ;\right.$ Alter: $\min =38 ; \max =68 ; \quad M W=51$; Median=49; Q1=44; Q3=56) der Teilnehmern machten keine Angaben zum Sport. (s. Abb. 15)

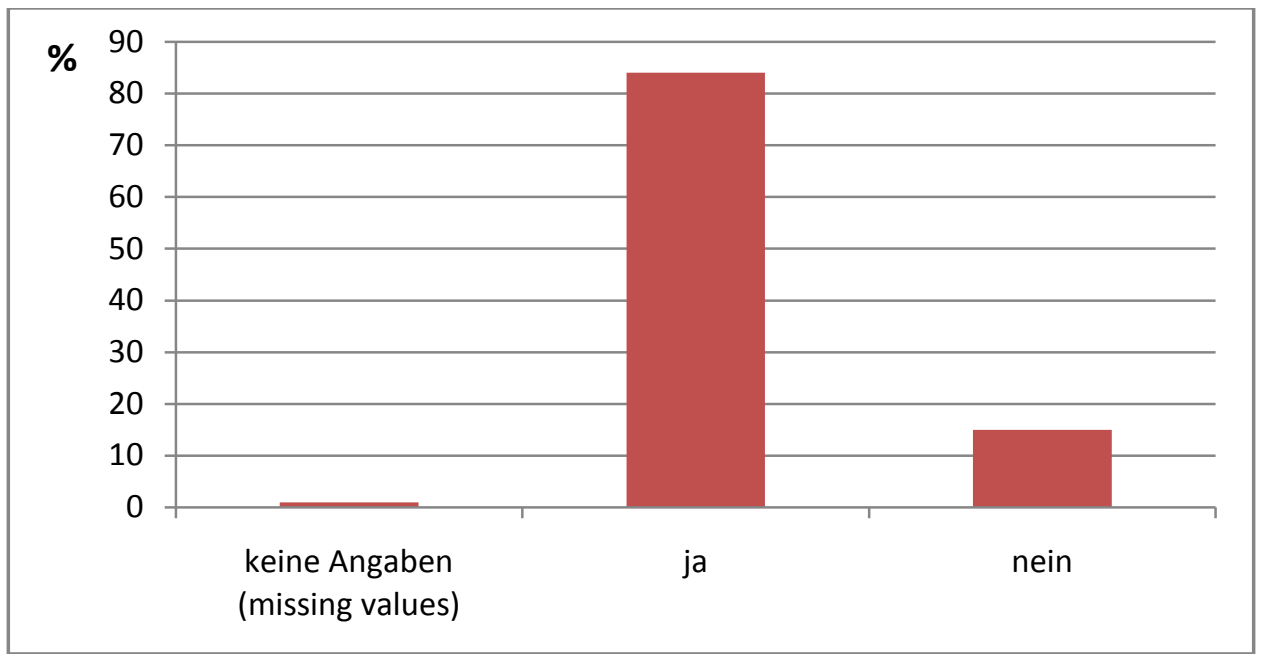

Abbildung 15: Darstellung des Gesamtkollektivs der Augenärzte/innen in Bezug auf das Ausüben von Sport (in \%), ja oder nein, sowie der fehlenden Angaben zu dieser Fragestellung (missing values) 


\subsubsection{Ergebnisse zu Zielgrößen}

\subsubsection{Zielgröße „Vorschäden“ unter den Augenärzten/innen}

In dem vorliegenden Gesamtkollektiv von 1807 Augenärzten bejahten hinsichtlich der Zielgröße Vorschäden an der Wirbelsäule zu haben 18\% ( $n_{\text {gesamt }}=325 ; n_{\text {männlich }}=168$; $\mathrm{n}_{\text {weiblich }}=152 ; \mathrm{n}_{\text {kAGeschlecht }}=5$; Alter: $\min =28 ; \max =74 ; \mathrm{MW}=50$; Median=50; $\mathrm{Q} 1=43 ; \mathrm{Q}=57$ ) der Teilnehmer.

Bei insgesamt 18\% $\quad\left(n_{\text {gesamt }}=325\right)$ der Teilnehmer mit Vorschäden, haben 70 Teilnehmer/innen mehrere Vorschäden die Verteilung der Lokalisationen der Vorschäden zeigt, dass die Skoliose mit 38\% $(n=146)$ die häufigste Vorerkrankung darstellt, gefolgt vom Morbus Scheuermann mit 25\% ( $n=96)$ und dem Diskus - Prolaps an der LWS mit 18\% $(n=70)$. Die anderen Vorerkrankungen, wie der Diskus - Prolaps an der HWS mit 7\% $(n=27)$, BWS mit 5\% ( $n=18)$ und dem lleosakralgelenk (ISG) mit 2\% $(n=7)$, sowie das Trauma mit 6\% $(n=25)$ spielen eine untergeordnete Rolle. (s. Abb. 16) Unter den Teilnehmern mit Vorschäden haben 86\% ( $n=278)$ aktuell Beschwerden, 14\% ( $n=45)$ sind ohne aktuelle Beschwerden und 1\% $(n=2)$ machten keine Angaben (missing values).

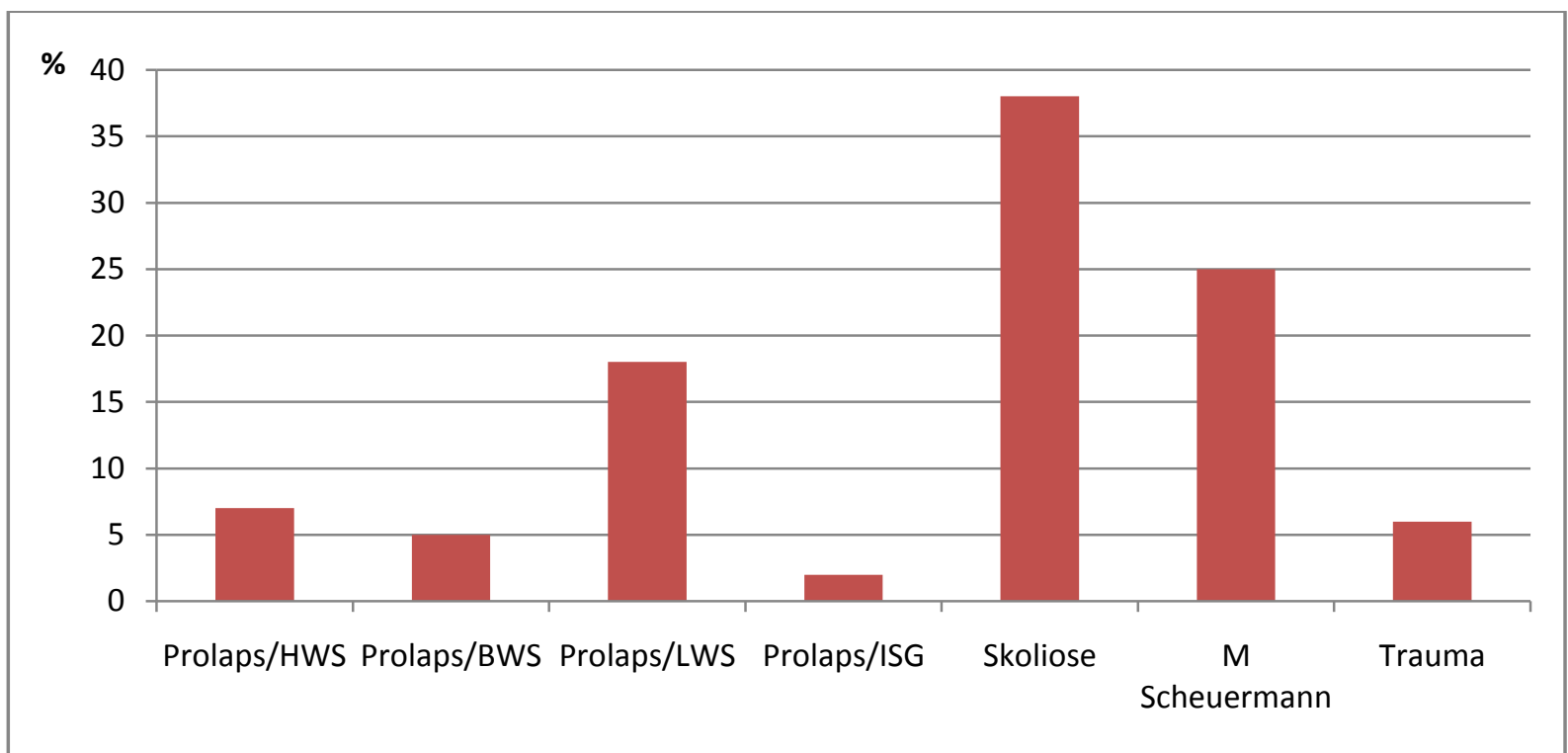

Abbildung 16: Vorschäden an der Wirbelsäule (in \%), bei denjenigen Augenärzten/innen, die Vorschäden angaben (n=325), stratifiziert nach der Lokalisation (Prolaps: HWS;BWS;LWS;ISG; Skoliose; M. Scheuermann; Trauma) 


\subsubsection{Zielgröße „aktuelle Beschwerden“ unter den Augenärzten/innen}

Von dem Gesamtkollektiv haben 81\% ( $n_{\text {gesamt }}=1464 ; \quad n_{\text {männlich }}=680 ; \quad n_{\text {weiblich }}=769$; $\mathrm{n}_{\text {kAGeschlecht }}=15 ;$ Alter: $\min =28 ; \quad \max =76 ; \quad M W=50 ; \quad$ Median=50; $\mathrm{Q1}=44 ; \quad \mathrm{Q}=57$ ) der Augenärzte/innen aktuell muskuloskeletale Beschwerden am Bewegungsapparat, davon sind 19\% $(n=278)$ Teilnehmer mit bestehenden Vorschäden. 71\% $(n=1037)$ der Teilnehmer mit aktuellen Beschwerden sind der Meinung, dass diese Beschwerden auf die Tätigkeit als Augenarzt zurückzuführen sind. $24 \% \quad(n=350)$ sehen die augenärztliche Tätigkeit als mögliche Ursache an und 4\% ( $n=52)$ glauben nicht, dass ein Zusammenhang besteht. $2 \%$ $(\mathrm{n}=25)$ machten keine Angaben (missing values) zur möglichen Ursache der bestehenden aktuellen muskuloskeletalen Beschwerden am Bewegungsapparat durch die augenärztliche Tätigkeit. (s. Abb. 17)

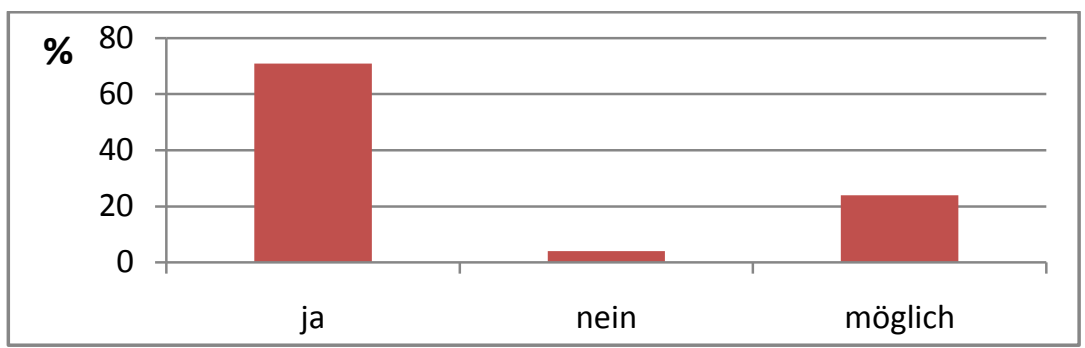

Abbildung 17: Augenärzte/innen mit aktuellen Beschwerden (in \%) und deren Meinung, dass diese Beschwerden auf die Tätigkeit als Augenarzt zurückzuführen sind

Die Teilnehmer mit aktuellen Beschwerden am muskuloskeletalen Bewegungsapparat konnten bei der Frage, welche Symptome Sie bei sich feststellen können, mehrere Symptome angeben. Sie gaben vorrangig Schmerzen mit 85\% ( $n=1245)$ und Verspannungen mit $83 \%(n=1221)$ an. Die Verteilung der weiteren Symptome lag bei $35 \%$ $(n=516)$ Bewegungseinschränkungen, 34\% ( $n=500)$ Parästhesien und $8 \% \quad(n=123)$ Instabilität. (s. Abb. 18)

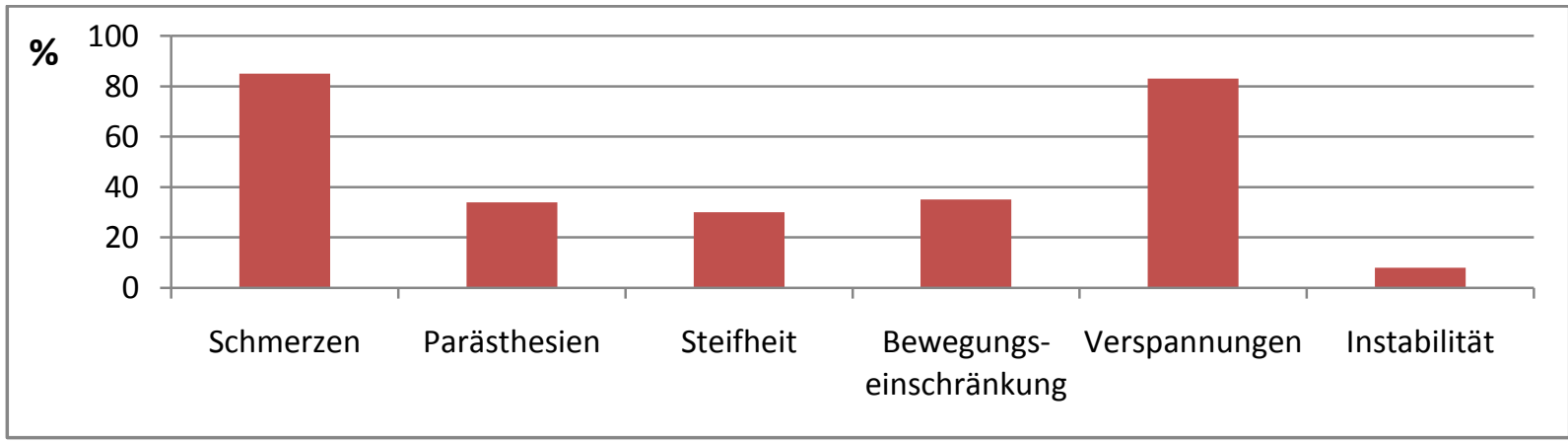

Abbildung 18: Augenärzte/innen mit aktuellen Beschwerden ( $n=1464)$ am muskuloskeletalen Bewegungsapparat (in \%), stratifiziert nach Symptomen (Schmerzen, Parästhesien, Steifheit, Bewegungseinschränkung, Verspannung und Instabilität) 
Die Beschwerdelokalisation bei den Teilnehmern mit aktuellen Beschwerden zeigt, dass die HWS mit 65\% ( $n=951)$ und die LWS mit 53\% ( $n=774)$ am häufigsten betroffen sind. Des Weiteren treten gehäuft Beschwerden an der Schulter mit 38\% $(n=557)$, Ausstrahlungen in die obere Extremität mit muskulären Beschwerden mit 26\% ( $n=384)$ und Beschwerden in der BWS mit 20\% ( $n=299)$ auf. Die anderen Beschwerdelokalisationen zeigen wie folgt eine geringere Häufigkeit unter den Teilnehmern, 19\% ( $n=277)$ mit Ausstrahlung in die obere Extremität mit neurologischen Beschwerden, 17\% ( $n=251)$ ISG, 14\% ( $n=207)$ Ausstrahlung in die untere Extremität mit neurologischen Beschwerden und 9\% $(n=139)$ Ausstrahlungen in die untere Extremität mit muskulären Beschwerden (s. Abb. 19)

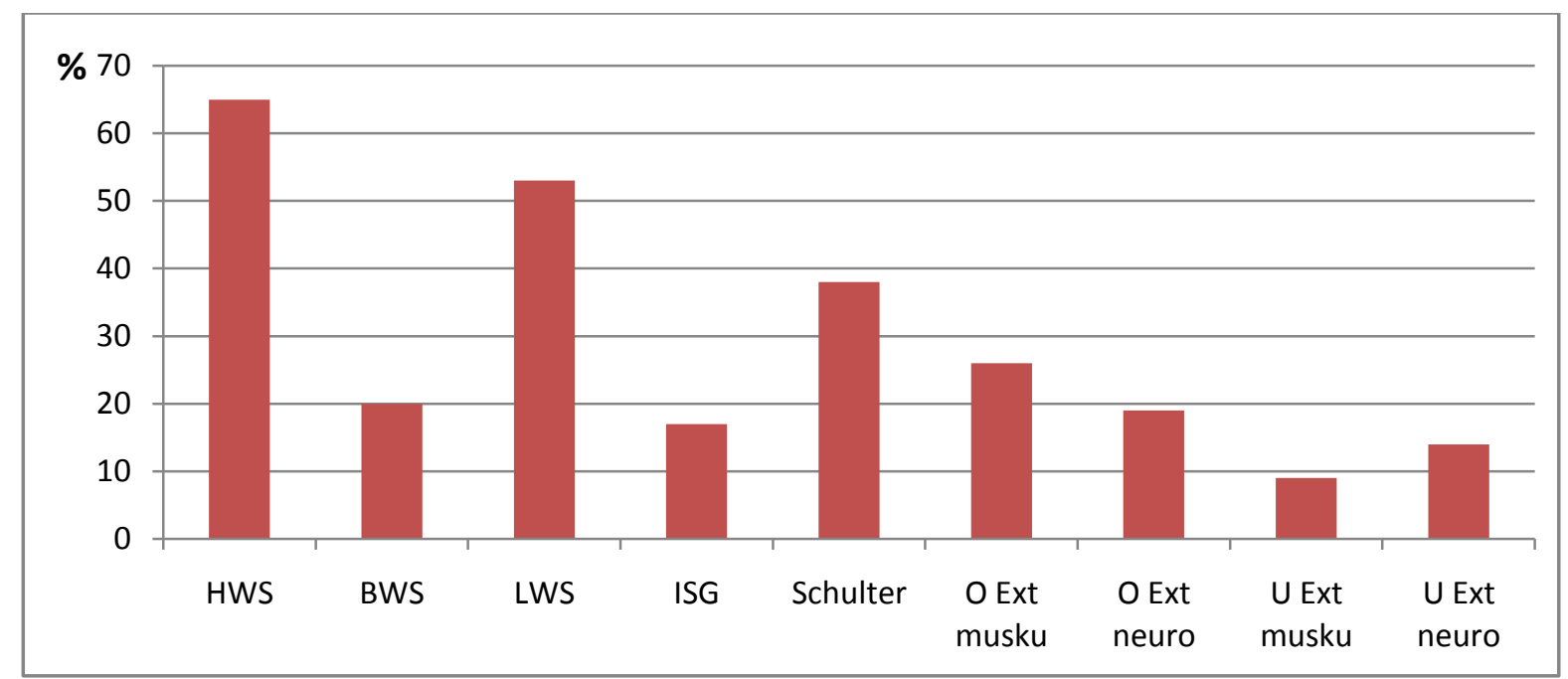

Abbildung 19: Augenärzte/innen mit aktuellen Beschwerden ( $n=1464)$ am muskuloskeletalen Bewegungsapparat (in \%) stratifiziert nach Beschwerdelokalisationen (Halswirbelsäule (HWS), Brustwirbelsäule (BWS), Lendenwirbelsäule (LWS), lleosakralgelenk (ISG), Schulter, obere Extremität mit muskulären Beschwerden (O Ext musku), obere Extremität mit neurologischen Beschwerden (O Ext neuro), untere Extremität mit muskulären Beschwerden (U Ext musku) und untere Extremität mit neurologischen Beschwerden (U Ext neuro)) 
3.2.2. Zielgröße „aktuelle Beschwerden“ und Einflußgrößen (Spezialgebiet, Tätigkeitsschwerpunkte, Sport) beeinflusst durch Alter und Geschlecht

Es besteht im Gesamtkollektiv ein mittleres Alter von 50 Jahren ( $\min =27$ Jahre; max=76 Jahre; Median=50; Q1=44; Q3=57) unter den Teilnehmern. (s. Abb. 20) Von den 1807 Augenärzten/innen hatten 7 Befragte keine Angaben (missing values) zum Alter gemacht und werden somit bei diesem Aspekt der Auswertung nicht berücksichtigt.

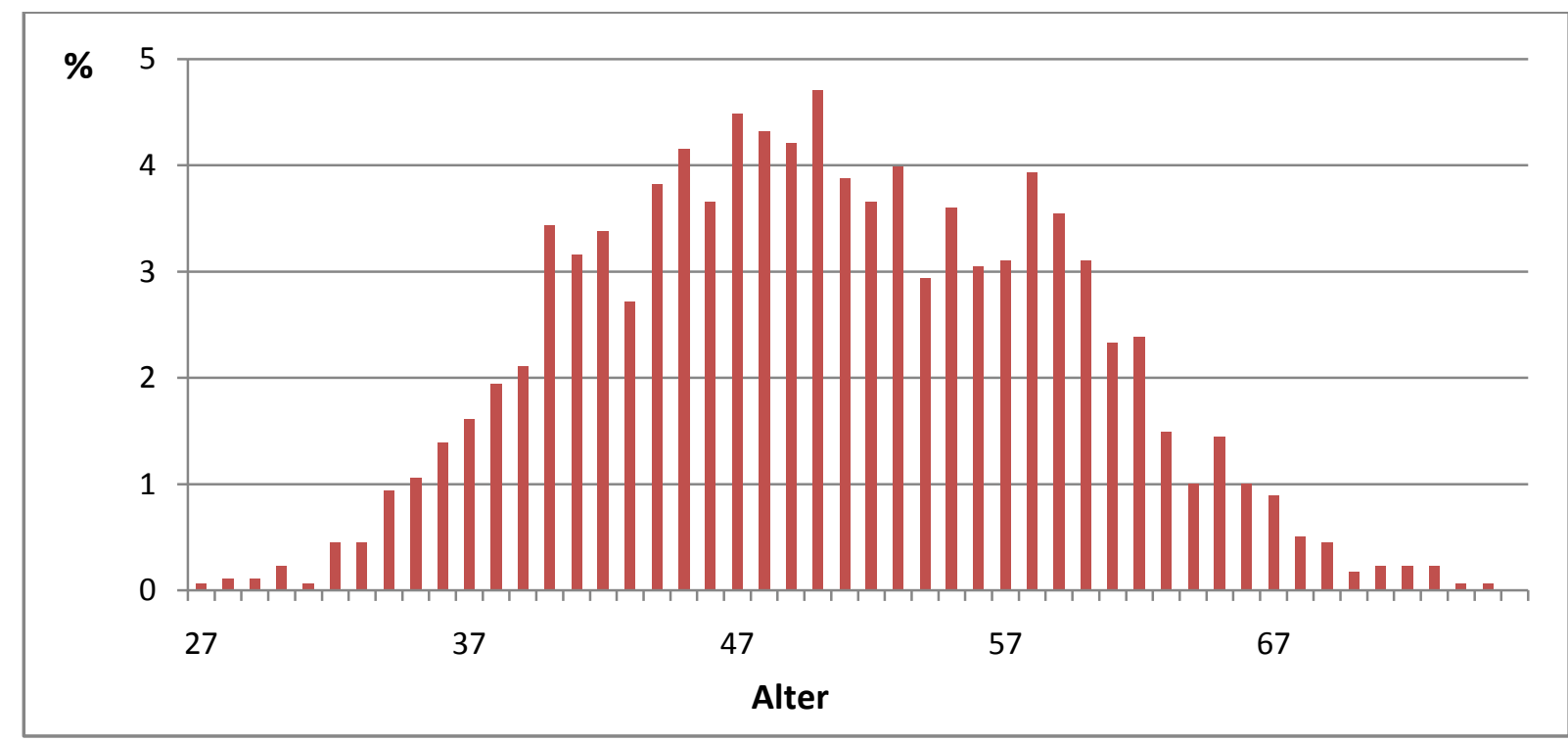

Abbildung 20: Altersverteilung der Augenärzte/innen im Gesamtkollektiv

Schlüsselt man nun das Gesamtkollektiv nach Geschlecht und Altersgruppen auf, zeigt sich, dass $100 \%$ der Teilnehmerinnen $(n=4)$ in der Altersgruppe 18 bis 29 angaben, Beschwerden zu haben. In der Altersgruppe 30 bis 39 sind die Anteile derer, die über Beschwerden klagten mit $69 \%(n=48)$ bei den männlichen Teilnehmern und $69 \%(n=78)$ bei den weiblichen Teilnehmern in etwa gleich hoch. Bei den 40- bis 49-Jährigen ist der Anteil der Frauen mit Beschwerden mit 86\% ( $n=317)$ höher als der Anteil der Männer mit Beschwerden, der 77\% ( $n=232)$ beträgt. Auch in der Altersgruppe der 50- bis 59-Jährigen liegt der Anteil der weiblichen Teilnehmern, die angaben, Beschwerden zu haben mit 87\% ( $n=280)$ etwas höher als bei den männlichen Teilnehmern, von denen in dieser Altersgruppe $79 \% \quad(n=257)$ Beschwerden bejahten. Ein ähnliches Bild ergibt sich auch bei den 60- bis 69-Jährigen, wo der Anteil der Augenärztinnen, die angaben, Beschwerden zu haben, mit $88 \%(n=83)$ ebenfalls höher ist als der Anteil von $81 \%(n=135)$ mit Beschwerden bei den Augenärzten. In der Altersgruppe der über 70-Jährigen schließlich gaben $83 \%(n=5)$ der befragten Frauen an, Beschwerden zu haben, während dies bei den befragten Männern dieser Altersgruppe auf $64 \%(n=7)$ zutraf. 
Insgesamt fällt bei der Betrachtung dieser Werte auf, dass die Anteile derer, die angaben, Beschwerden zu haben, bei den Teilnehmern aller Altersgruppen deutlich über 50\% liegt und die befragten Augenärztinnen tendenziell noch häufiger von Beschwerden betroffen waren, als ihre männlichen Kollegen. (s. Abb. 21)

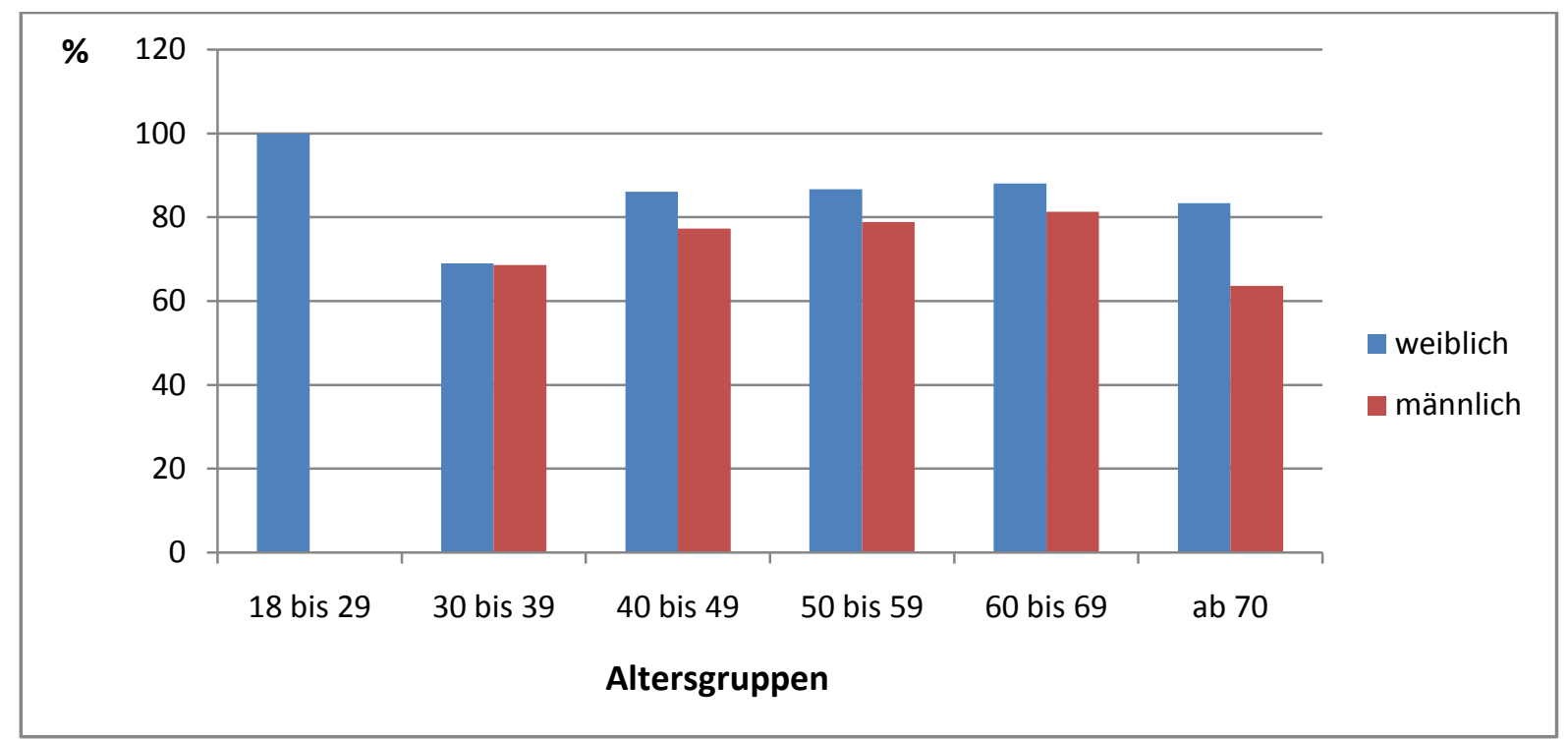

Abbildung 21: Augenärzte/innen mit aktuellen Beschwerden (in \%), aufgeteilt in weiblich und männlich in den einzelnen Altersgruppen ( 18-29 Jahre, 30-39 Jahre, 40-49 Jahre, 50-59 Jahre, 60-69 Jahre und ab 70 Jahre)

Dass Augenärzte und Augenärztinnen tatsächlich häufiger als die Normalbevölkerung von Beschwerden am muskuloskeletalen Bewegungsapparat betroffen sind, zeigen die im Folgenden dargestellten Grafiken. Vergleicht man die zuvor genannten Daten für die muskuloskeletalen Beschwerden bei weiblichen und männlichen Augenärzten mit den Daten für die Normalbevölkerung, die ein telefonisches Gesundheitssurvey des Robert - Koch Instituts (RKI) im Jahr 2003 (RKI 2006) ergeben hat, wird insgesamt deutlich, dass die Anteile derer, die angeben, Beschwerden zu haben, bei den Augenärzten/innen in allen Altersgruppen höher sind, als in der Normalbevölkerung.

So zeigt sich bei den weiblichen Teilnehmern beider Umfragen in der Altersgruppe der 18bis 29-Jährigen, dass in dieser Altersgruppe 100\% der Augenärztinnen angaben, Beschwerden zu haben, während in der Normalbevölkerung nur 22\% über Beschwerden klagten. In der Altersgruppe der 30- bis 39-Jährigen sind 69\% unter den Augenärztinnen und $23 \%$ in der Normalbevölkerung betroffen. Bei den 40- bis 49-Jährigen, besteht ein Anteil von $86 \%$ bei den Augenärztinnen und $25 \%$ bei der Normalbevölkerung mit Beschwerden gegenüber. In der Altersgruppe der 50 - bis 59-Jährigen traten bei $87 \%$ der Augenärztinnen und bei $33 \%$ in der Normalbevölkerung Beschwerden auf. Es ergibt sich bei der Altersgruppe der 60 - bis 69-Jährigen folgende Verteilung: So sind $88 \%$ der Augenärztinnen und $29 \%$ in 
der Normalbevölkerung betroffen. Bei der Altersgruppe ab 70 Jahren, ist auch hier deutlich zu sehen, dass die Augenärztinnen mit 83\% höher betroffen sind, als die Frauen in der Normalbevölkerung mit 31\%. (s. Abb. 22)

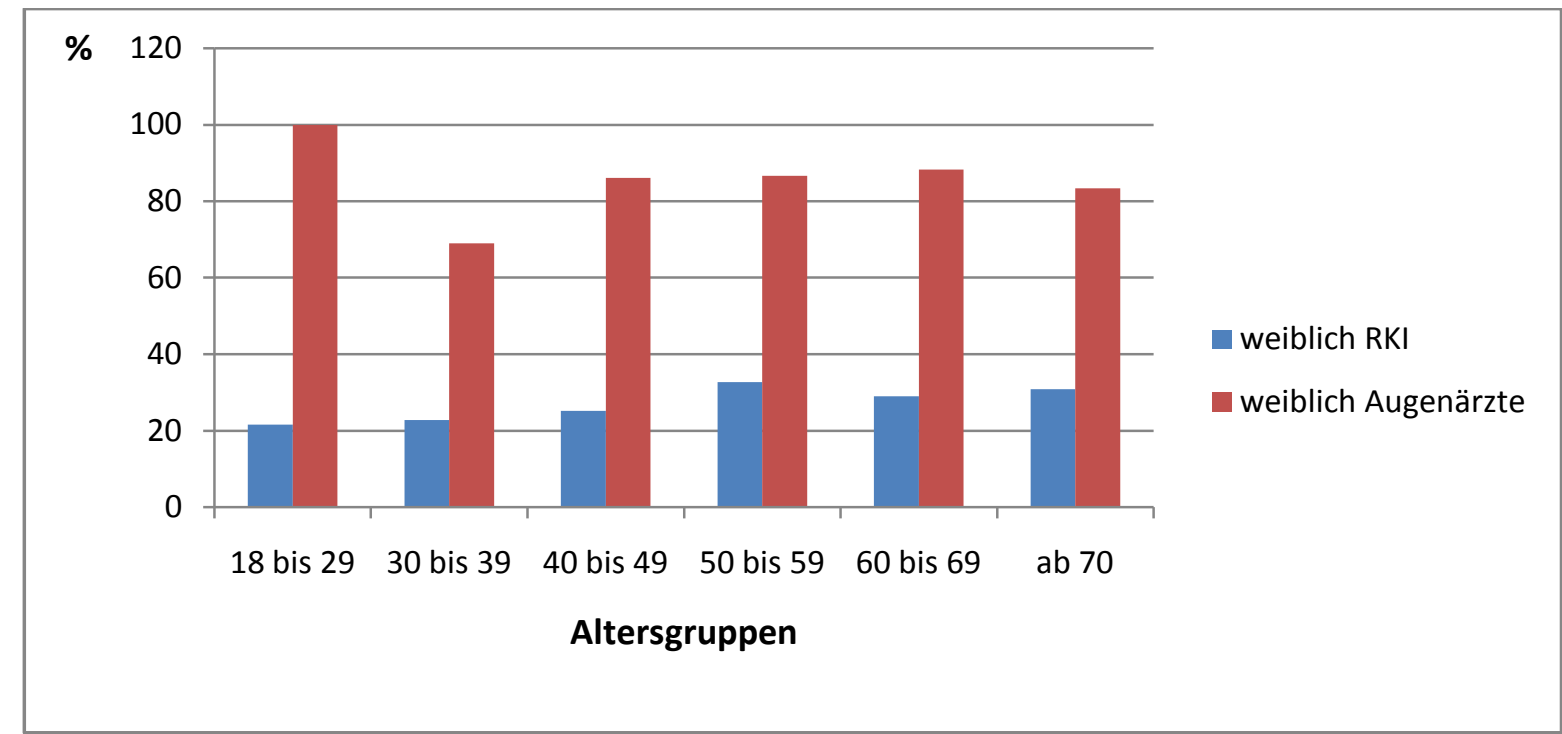

Abbildung 22: Vergleich der Augenärztinnen mit aktuellen Beschwerden (in \%), mit der weiblichen Normalbevölkerung (in \%) (welche in einer Studie des Robert-Koch-Instituts (RKI) 2003 zu bestehenden Beschwerden am muskoloskeletalen Bewegungsapparat befragt wurden) in den einzelnen Altersgruppen ( 18-29 Jahre, 30-39 Jahre, 40-49 Jahre, 50-59 Jahre, 60-69 Jahre und ab 70 Jahre) 
Ähnlich wie schon bei den weiblichen Teilnehmern ersichtlich wurde, zeigt sich auch bei den männlichen Teilnehmern beider Umfragen, dass Augenärzte häufiger von Beschwerden am muskuloskeletalen Bewegungsapparat betroffen sind als männliche Teilnehmer aus der Normalbevölkerung. So steht in der Altersgruppe der 30- bis 39-Jährigen ein Anteil der Männer mit Beschwerden in der Normalbevölkerung von 15\% einen wesentlich höheren Anteil, nämlich 69\% an Augenärzten mit Beschwerden gegenüber. Bei den 40- bis 49Jährigen gaben $16 \%$ der vom RKI befragten Männer aus der Normalbevölkerung an, unter Beschwerden zu leiden, während diese bei den Augenärzten in dieser Altersgruppe einen Anteil von $77 \%$ ausmachen. In der Altersgruppe der 50- bis 59-Jährigen steht ebenso ein wesentlich höherer Anteil von 79\% der befragten Augenärzte mit Beschwerden einem vergleichsweise geringem Anteil von $25 \%$ von Männern aus der Normalbevölkerung, die angaben, Beschwerden zu haben, gegenüber. Bei den 60- bis 69-Jährigen Teilnehmern aus der Normalbevölkerung klagten $21 \%$, während mit einem Anteil von $81 \%$ ein etwa viermal so großer Anteil der Augenärzte muskuloskeletale Beschwerden bejahte. In der Altersgruppe ab 70 Jahren gaben 22\% aus der Normalbevölkerung an, unter Beschwerden zu leiden, während hier 64\% der Augenärzte über Beschwerden klagten. (s. Abb. 23)

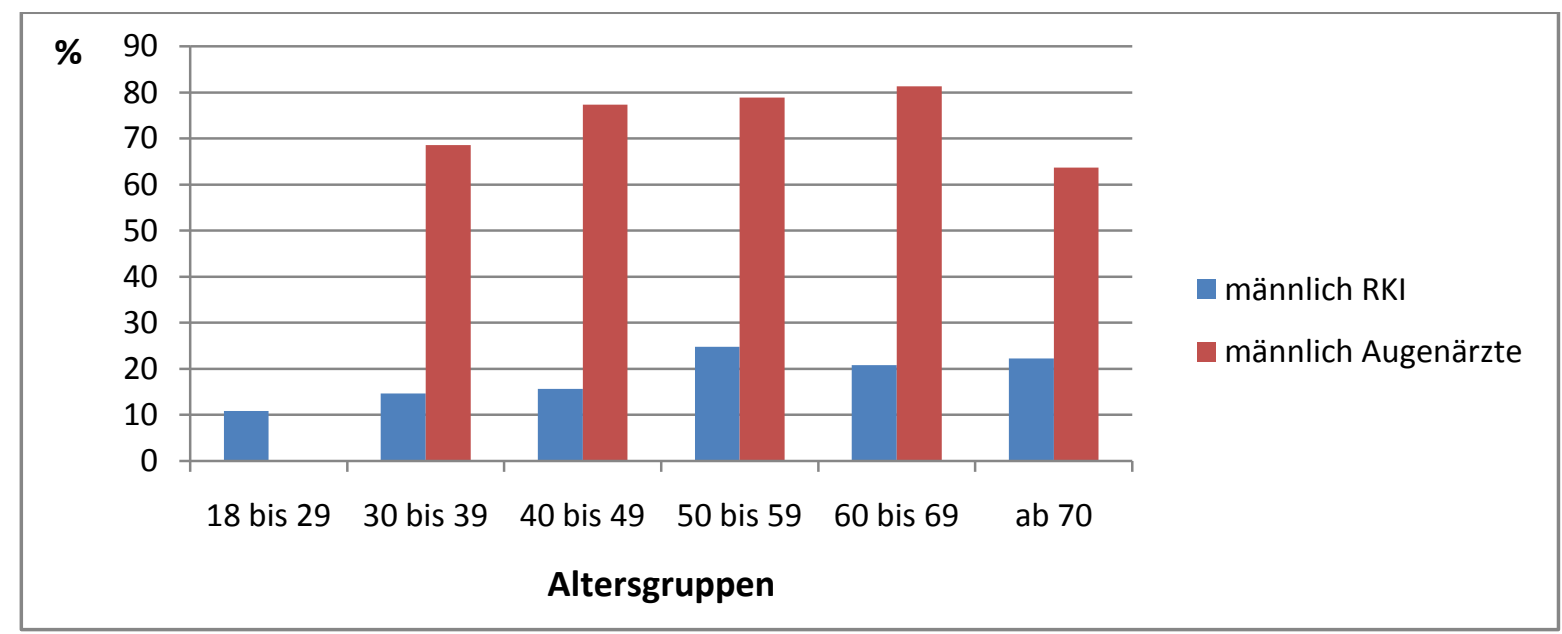

Abbildung 23: Vergleich der Augenärzte mit aktuellen Beschwerden (in \%), mit der männlichen Normalbevölkerung (in \%) (welche in einer Studie des Robert-Koch-Instituts (RKI) 2003 zu bestehenden Beschwerden am muskoloskeletalen Bewegungsapparat befragt wurden) in den einzelnen Altersgruppen ( 18-29 Jahre, 30-39 Jahre, 40-49 Jahre, 50-59 Jahre, 60-69 Jahre und ab 70 Jahre)

Betrachtet man die Altersverteilung unter den weiblichen Teilnehmern mit aktuellen Beschwerden in Bezug auf die Einflussgröße Spezialgebiet in der Augenheilkunde, ergibt sich, dass in allen Altersgruppen die meisten Augenärztinnen kein Spezialgebiet haben. So entfallen auf diejenigen, die „kein Spezialgebiet“ ankreuzten, 100\% $(n=4)$ der Altersgruppe der 18- bis 29-Jährigen, 73\% ( $n=82)$ der Altersgruppe der 30- bis 39-Jährigen, 76\% $(n=273)$ 
der Altersgruppe der 40- bis 49-Jährigen, 82\% ( $n=261)$ der Altersgruppe der 50- bis 59Jährigen, 89\% $(n=75)$ der Altersgruppe der 60- bis 69-Jährigen und 67\% ( $n=4)$ der Altersgruppe ab 70 Jahren. Die Altersverteilung der Teilnehmerinnen im Spezialgebiet der „Strabologie“ zeigt, dass dieses Gebiet eher von älteren Augenärztinnen ausgeführt wird. In den Altersgruppen lag die Verteilung wie folgt: Bei $5 \%(n=6)$ in der Altersgruppe der 30- bis 39-Jährigen, 10\% ( $n=34)$ in der Altersgruppe der 40- bis 49-Jährigen, 11\% $(n=36)$ in der Altersgruppe der 50- bis 59-Jährigen, $5 \%(n=4)$ in der Altersgruppe der 60- bis 69-Jährigen und $33 \%(n=2)$ in der Altersgruppe ab 70 Jahren. Bei der Altersgruppe der 18- bis 29Jährigen liegen keine Ergebnisse vor. Von der Gesamtzahl aller, die im Spezialgebiet „Vorderabschnitt“ tätig sind, befinden sich die meisten in den jüngeren Altersgruppen, dabei entfallen $18 \%(n=20)$ auf die Altersgruppe der 30- bis 39-Jährigen, 11\% $(n=38)$ auf die Altersgruppe der 40- bis 49-Jährigen, 4\% $(n=14)$ auf die Altersgruppe der 50- bis 59-Jährigen und $5 \%(n=4)$ auf die Altersgruppe der 60- bis 69-Jährigen. In den Altersgruppen der 18- bis 29-Jährigen und ab 70 Jahren liegen keine Ergebnisse vor. Im Spezialgebiet „Hinterabschnitt“ gibt es eine Verteilung von 4\% $(n=4)$ in der Altersgruppe der 30- bis 39Jährigen, 4\% $(n=13)$ in der Altersgruppe der 40- bis 49-Jährigen, 2\% $(n=7)$ in der Altersgruppe der 50- bis 59-Jährigen und 1\% $(n=1)$ in der Altersgruppe der 60- bis 69Jährigen. Wie auch in dem Spezialgebiet „Vorderabschnitt“, gab es auch in dem Spezialgebiet „Hinterabschnitt“ keine Beteiligung der Teilnehmerinnen in den Altersgruppen der 18- bis 29-Jährigen und ab 70 Jahren. (s. Abb. 24)

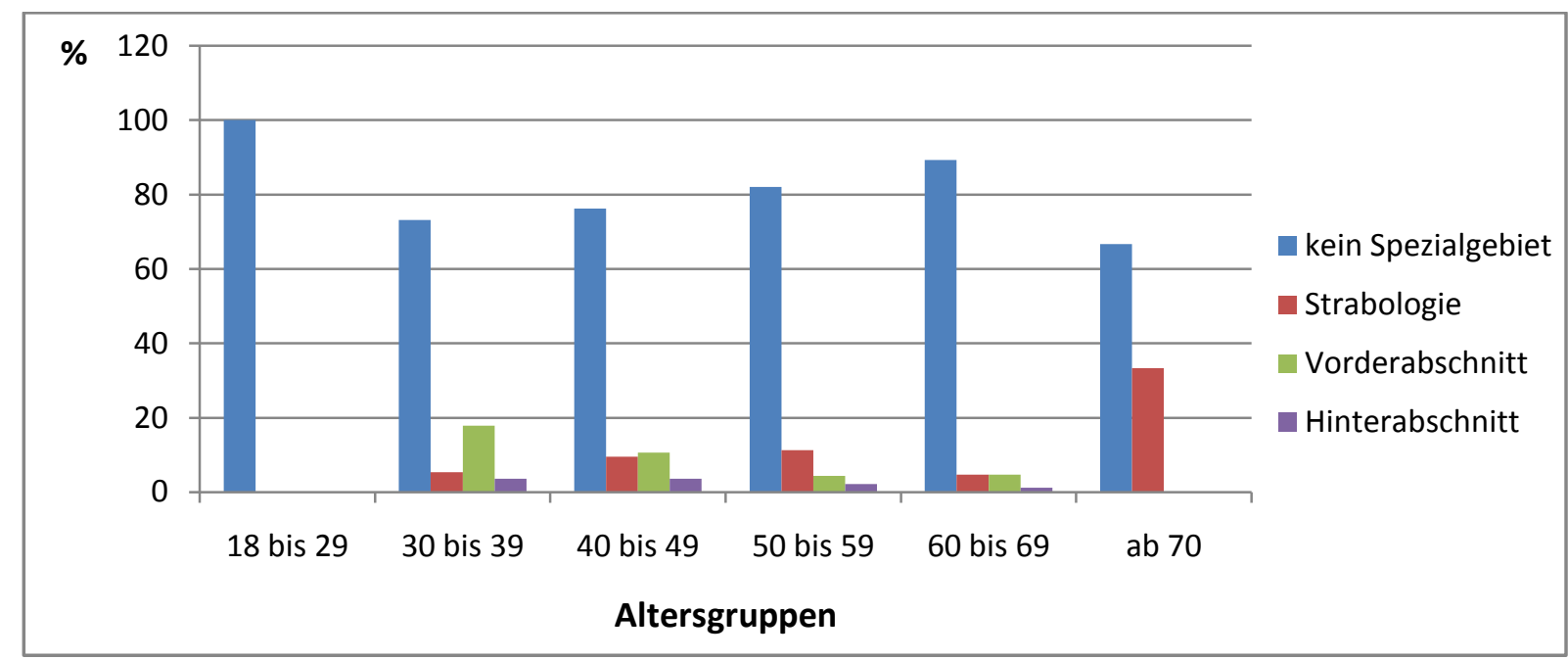

Abbildung 24: weibliche Teilnehmer mit aktuellen Beschwerden (in \%), in den einzelnen Altersgruppen ( 18-29 Jahre, 3039 Jahre, 40-49 Jahre, 50-59 Jahre, 60-69 Jahre und ab 70 Jahre), stratifiziert nach den Spezialgebieten (Stabologie, Vorderabschnitt, Hinterabschnitt und kein bestehenden Spezialgebiet) 
Eine ähnliche Verteilung zeigt sich auch in Bezug auf die Verteilung der männlichen Teilnehmer mit aktuellen Beschwerden auf die einzelnen Spezialgebiete: Der Anteil der Teilnehmer die „kein Spezialgebiet“ angaben, liegt bei den 18- bis 19-Jährigen bei $100 \%$ $(n=1)$, bei den 30 - bis 39-Jährigen bei $50 \%(n=35)$, bei den 40 - bis 49 -Jährigen bei $53 \%$ $(n=155)$, mit $72 \%(n=227)$ bei den 50 - bis 59-Jährigen, sowie mit $67 \%(n=106)$ bei den 60 bis 69-Jährigen und bei den Teilnehmern ab 70 Jahren bei $80 \%(n=8)$. In dem Spezialgebiet "Strabologie“ ist genauso wie bei den weiblichen Augenärzten zu erkennen, dass dieses Gebiet eher von den älteren Augenärzten ausgeführt wird. So ergibt sich eine Altersverteilung von 7\% $(n=5)$ in der Altersgruppe der 30- bis 39-Jährigen, 8\% $(n=23)$ in der Altersgruppe der 40- bis 49-Jährigen, $8 \%(n=25)$ in der Altersgruppe der 50- bis 59-Jährigen, $15 \%(n=23)$ in der Altersgruppe der 60- bis 69-Jährigen und $20 \%(n=2)$ in der Altersgruppe ab 70 Jahren. Für die Altersgruppe der 18- bis 29-Jährigen liegen keine Ergebnisse vor. In Bezug auf die Gesamtzahl aller, die im Spezialgebiet „Vorderabschnitt“ tätig sind, lässt sich feststellen, dass sich die meisten der Augenärzte in den jüngeren Altersgruppen befinden. Es entfallen 34\% ( $n=24)$ auf die Altersgruppe der 30- bis 39-Jährigen, 32\% $(n=95)$ auf die Altersgruppe der 40 - bis 49-Jährigen, $16 \%(n=50)$ auf die Altersgruppe der 50- bis 59Jährigen und $12 \%(n=19)$ auf die Altersgruppe der 60- bis 69-Jährigen. Für die Altersgruppen der 18- bis 29-Jährigen und ab 70 Jahren liegen keine Ergebnisse vor. Unter denjenigen, die im Spezialgebiet „Hinterabschnitt“ tätig sind, sind 9\% $(n=6)$ der Altersgruppe der 30- bis 39Jährigen, 7\% $(n=20)$ der Altersgruppe der 40- bis 49-Jährigen, 5\% $(n=15)$ der Altersgruppe der 50 - bis 59 -Jährigen und $7 \%(n=11)$ der Altersgruppe der 60- bis 69-Jährigen zuzuordnen. In der Altersgruppe der 18- bis 29-Jährigen und ab 70 Jahren liegen keine Ergebnisse vor. (s. Abb. 25)

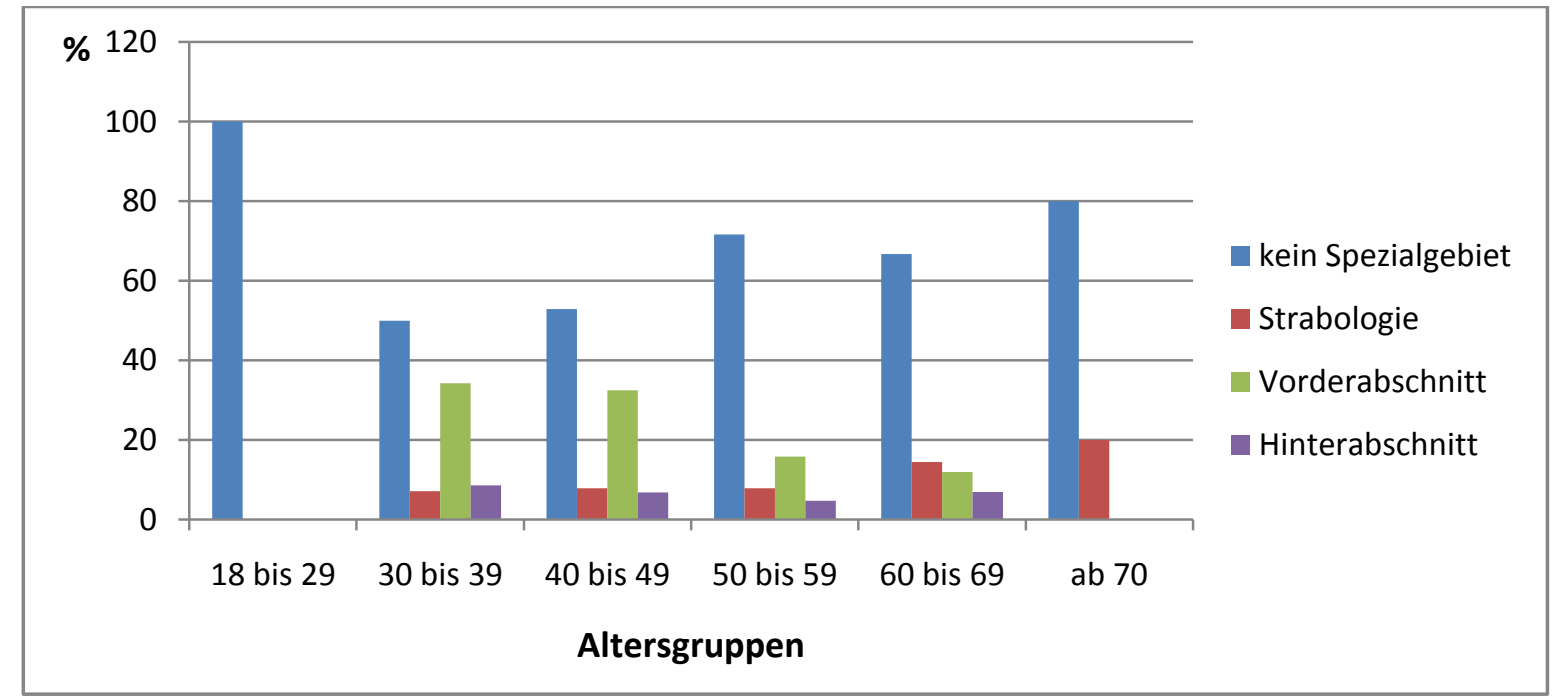

Abbildung 25: männliche Teilnehmer mit aktuellen Beschwerden (in \%), in den einzelnen Altersgruppen ( 18-29 Jahre, 3039 Jahre, 40-49 Jahre, 50-59 Jahre, 60-69 Jahre und ab 70 Jahre), stratifiziert nach den Spezialgebieten (Stabologie, Vorderabschnitt, Hinterabschnitt und kein bestehenden Spezialgebiet) 
In der Verteilung der Altersgruppen, in Bezug auf die Einflussgröße Tätigkeitsschwerpunkte „konservativ“ und „operativ“ unter den weiblichen Augenärzten mit aktuellen Beschwerden. Ist ersichtlich, dass die Mehrzahl der Augenärztinnen im „rein konservativen“ und „konservativen > operativen“ Bereich tätig sind. Es ergibt sich daraus eine Verteilung in den einzelnen Altersgruppen wie folgt: In der Altersgruppe der 18- bis 29-Jährigen sind 100\% $(n=4)$ der Augenärztinnen im „rein konservativen“ Bereich tätig sind. Es entfallen 46\% $(n=50)$ auf den „rein konservativen“ Bereich, 45\% ( $n=49)$ auf den „konservativen > operativen“ Bereich und 9\% $(n=10)$ auf den „konservativen < operativen“ Bereich in der Altersgruppe der 30- bis 39- Jährigen. Im ,rein operativen“ Bereich sind in dieser Altersgruppe keine Augenärztinnen vertreten. Bei der Altersgruppe der 40- bis 49-Jährigen arbeiten 40\% ( $n=141)$ im „rein konservativen“ Bereich, 48\% ( $n=170)$ im „konservativen > operativen“ Bereich, 10\% ( $n=37)$ im „konservativen < operativen“ Bereich und $2 \%(n=8)$,rein operativen“ Bereich. Eine ähnliche Verteilung zeigt sich auch in der Altersgruppe der 50- bis 59-Jährigen, wo $45 \%(n=139)$ im „rein konservativen“ Bereich, 48\% ( $n=150)$ im „konservativen > operativen“ Bereich, 5\% ( $n=15)$ im „konservativen < operativen“ Bereich und 3\% $(n=8)$ im „rein operativen“ Bereich der Teilnehmerinnen tätig sind. In der Altersgruppe der 60- bis 69Jährigen arbeiten 54\% ( $n=49)$ im „rein konservativen“ Bereich, 38\% ( $n=35)$ im „konservativen > operativen“ Bereich und $8 \%(n=7)$ der Teilnehmerinnen im „konservativen < operativen“ Bereich. Die Verteilung in der Altersgruppe ab 70 Jahren ergibt, dass $67 \%(n=4)$ im „rein konservativen“ Bereich, 17\% $(n=1)$ im „konservativen > operativen“ Bereich und 17\% $(n=1)$ im „konservativen < operativen“ Bereich tätig sind. In den Beiden letzten Altersgruppen arbeiten keine Augenärztinnen im „rein operativen“ Bereich. (s. Abb. 26)

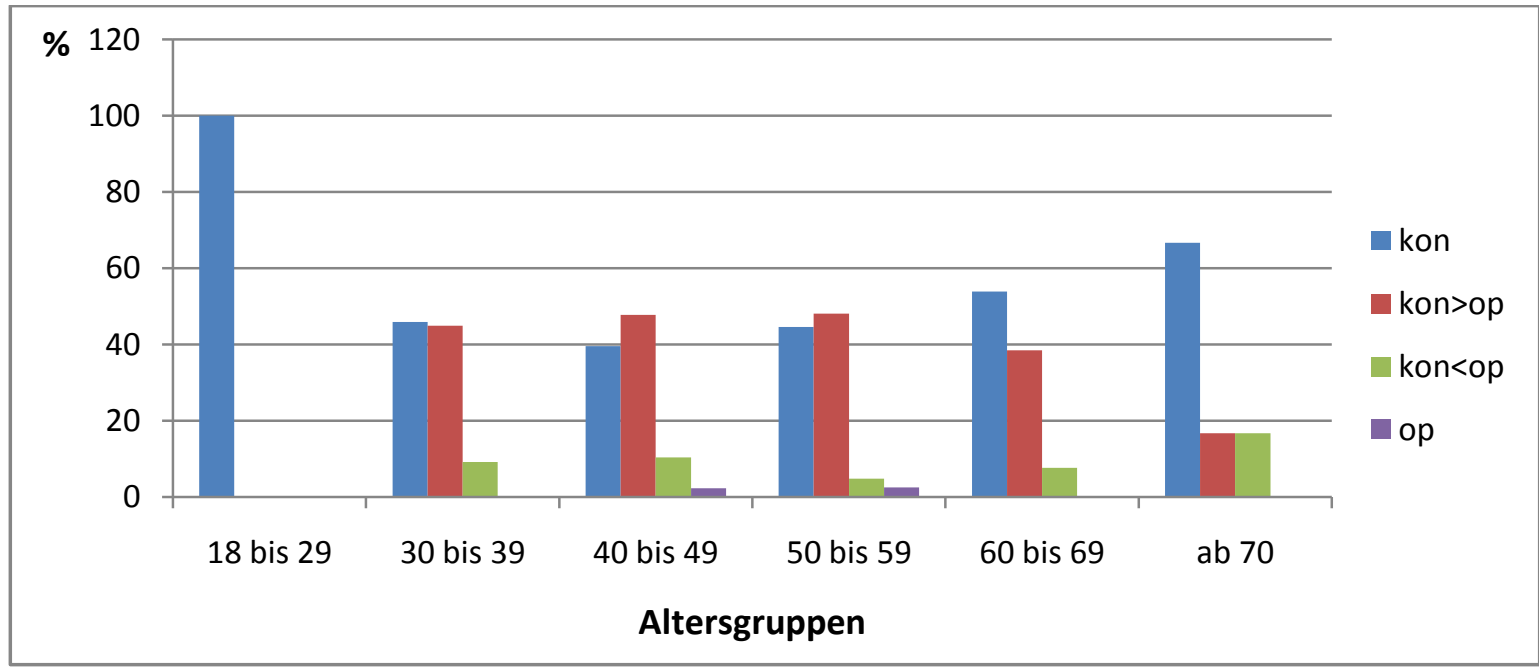

Abbildung 26: weibliche Teilnehmer mit aktuellen Beschwerden (in \%), in den einzelnen Altersgruppen ( 18-29 Jahre, 3039 Jahre, 40-49 Jahre, 50-59 Jahre, 60-69 Jahre und ab 70 Jahre), stratifiziert nach den Tätigkeitsschwerpunkten (rein konservativ, konservativ>operativ, konservativ<operativ und rein operativ) 
Ein anderes Bild zeigt die Verteilung der Altersgruppen, in Bezug auf die ,Einflussgröße Tätigkeitsschwerpunkte „konservativ“ und „operativ“ unter den männlichen Augenärzten mit aktuellen Beschwerden, im Gegensatz zu den weiblichen Augenärzten. In fast allen Altersgruppen ist ersichtlich, dass die männlichen Augenärzte vermehrt in Kombination zwischen „konservativem“ und „operativem“ Bereich arbeiten aber der Schwerpunkt überwiegend im „operativen“ Bereich liegt. In den einzelnen Altersgruppen ergibt sich eine Verteilung wie folgt: So sind in der Altersgruppe der 18- bis 29-Jährigen alle befragten Augenärzte im „rein konservativen“ Bereich tätig. Bei der Altersgruppe der 30- bis 39Jährigen, sind 13\% ( $n=9)$ im „rein konservativen“ Bereich, 57\% ( $n=39)$ im „konservativen > operativen“ Bereich, 29\% ( $n=20)$ im „konservativen < operativen“ Bereich und 1\% $(n=1)$ im „rein operativen“ Bereich tätig. In der Altersgruppe der 40- bis 49-Jährigen arbeiten 20\% $(n=57)$ im „rein konservativen“ Bereich, 51\% ( $n=148)$ im „konservativen > operativen“ Bereich, 25\% ( $n=72)$ im „konservativen < operativen“ Bereich und 5\% ( $n=14)$ im „rein operativen“ Bereich. Von den Teilnehmern, die in der Altersgruppe der 50- bis 59-Jährigen sind, entfallen 24\% ( $n=75)$ auf den „rein konservativen“ Bereich, 59\% ( $n=187)$ auf den „konservativen > operativen“ Bereich, 14\% ( $n=45)$ auf den „konservativen < operativen“ Bereich und $3 \%(n=8)$ auf den „rein operativen“ Bereich. Die Altersgruppe der 60- bis 69Jährigen verteilt sich wie folgt auf die einzelnen Tätigkeitsschwerpunkte: $19 \% \quad(n=30)$ der Teilnehmer sind im „rein konservativen“ Bereich, 68\% ( $n=107)$ im „konservativen > operativen“ Bereich, 11\% ( $n=18)$ im „konservativen < operativen“ Bereich und $2 \%(n=3)$ im „rein operativen“ Bereich aktiv. In der letzten Altersgruppe der ab 70 Jahren, sind $18 \%(n=2)$ im „rein konservativen“ Bereich, 46\% $(n=5)$ im „konservativen > operativen“ Bereich und 36\% $(n=4)$ der Augenärzte im „konservativen < operativen“ Bereich tätig. Im „rein operativen“ Arbeitsbereich liegen keine Ergebnisse vor. (s. Abb. 27)

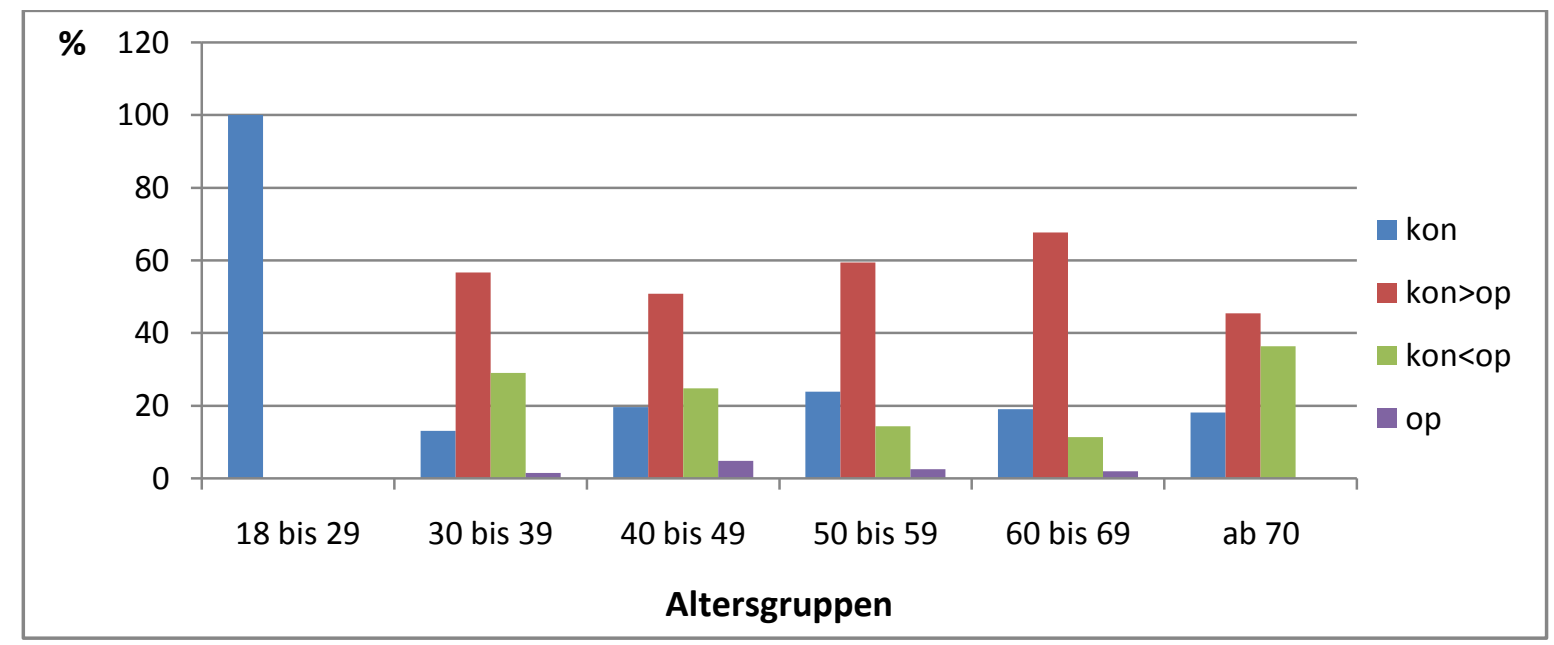

Abbildung 27: männliche Teilnehmer mit aktuellen Beschwerden (in \%), in den einzelnen Altersgruppen ( 18-29 Jahre, 30 39 Jahre, 40-49 Jahre, 50-59 Jahre, 60-69 Jahre und ab 70 Jahre), stratifiziert nach den Tätigkeitsschwerpunkten (rein konservativ, konservativ>operativ, konservativ<operativ und rein operativ) 
Des Weiteren wurde nach der Verteilung des Einflussfaktors "Sport“ in den einzelnen Altersgruppen der Augenärzte/innen mit aktuellen Beschwerden geschaut. In allen Altersgruppen ist ersichtlich, dass die Mehrzahl der Augenärzte/innen mit aktuellen Beschwerden Sport treiben. Unter den weiblichen Augenärzten liegt der Prozentsatz der Sport treibenden weit über $60 \%$ in allen Altersgruppen. Bei den 18- bis 29-Jährigen treiben $100 \%(n=4)$ der Augenärztinnen Sport. Folgende Verteilung ergibt sich in den anderen Altersgruppen der Augenärztinnen: 80\% $(n=90)$ treiben Sport und 20\% ( $n=22)$ verneinten Sport zu betreiben in der Altersgruppe der 30- bis 39-Jährigen. Unter den 40- bis 49Jährigen treiben $86 \%(n=315)$ Sport und 14\% $(n=50)$ nicht. Bei der Altersgruppe der 50 - bis 59-Jährigen betreiben $85 \%(n=275)$ Sport und 15\% ( $n=47)$ keinen Sport. In der Altersgruppe der 60- bis 69-Jährigen üben 75\% ( $n=71)$ Sport aus und 25\% $(n=23)$ betreiben keinen Sport. Bei der Altersgruppe der über 70- Jährigen liegt der Prozentsatz derer die Sport aus üben bei $67 \%(n=4)$ und $33 \%(n=2)$ betreiben keinen Sport. (s. Abb. 28$)$

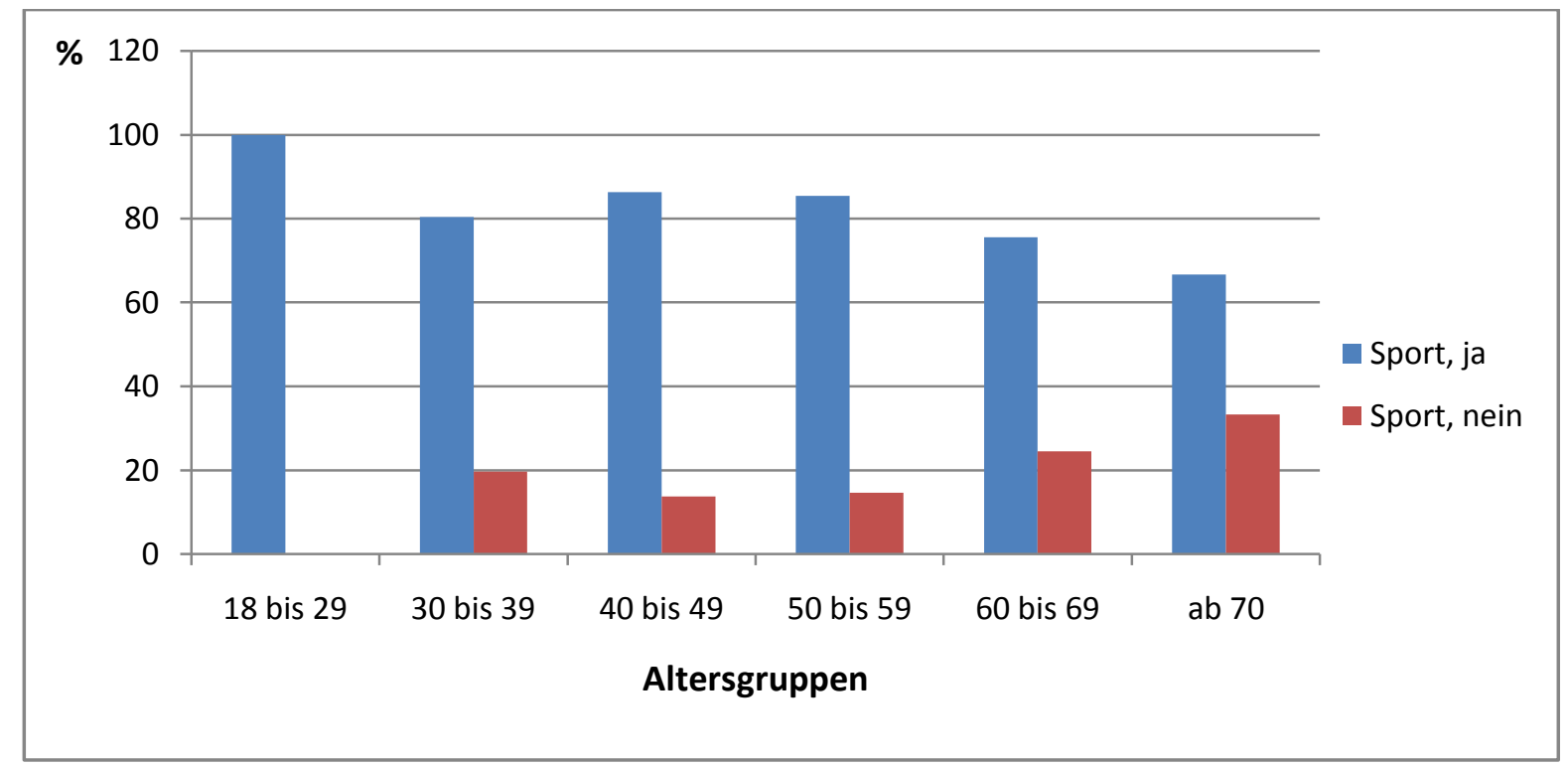

Abbildung 28: weibliche Teilnehmer mit aktuellen Beschwerden (in \%), in den einzelnen Altersgruppen ( 18-29 Jahre, 3039 Jahre, 40-49 Jahre, 50-59 Jahre, 60-69 Jahre und ab 70 Jahre), stratifiziert nach dem Einflussfaktor Sport (betreiben von Sport und nicht betreiben von Sport) 
Unter den männlichen Augenärzten mit aktuellen Beschwerden zeigt sich noch ein höherer Prozentsatz der Sport treibenden in den einzelnen Altersgruppen gegenüber den weiblichen Augenärzten. So ergibt sich das in der Altersgruppe der 18- bis 29-Jährigen 100\% $(n=1)$ der Augenärzte Sport treiben. Unter den 30- bis 39-Jährigen treiben 87\% ( $n=61)$ Sport und 13\% $(n=9)$ nicht. In der Altersgruppe der 40- bis 49-Jährigen sind es 87\% ( $n=262)$ die Sport betreiben und 13\% $(n=38)$ betreiben keinen Sport. Bei der Altersgruppe der 50- bis 59Jährigen betreiben 82\% ( $n=267)$ Sport und 18\% $(n=57)$ keinen Sport. In der Altersgruppe der 60- bis 69-Jährigen üben $83 \%(n=138)$ Sport aus und 17\% ( $n=28)$ machen keinen Sport. Eine ähnliche Verteilung liegt auch in der Altersgruppe der ab 70 Jährigen vor, 82\% $(n=9)$ der Augenärzte betreiben Sport und 18\% $(n=2)$ betreiben keinen Sport. (s. Abb. 29)

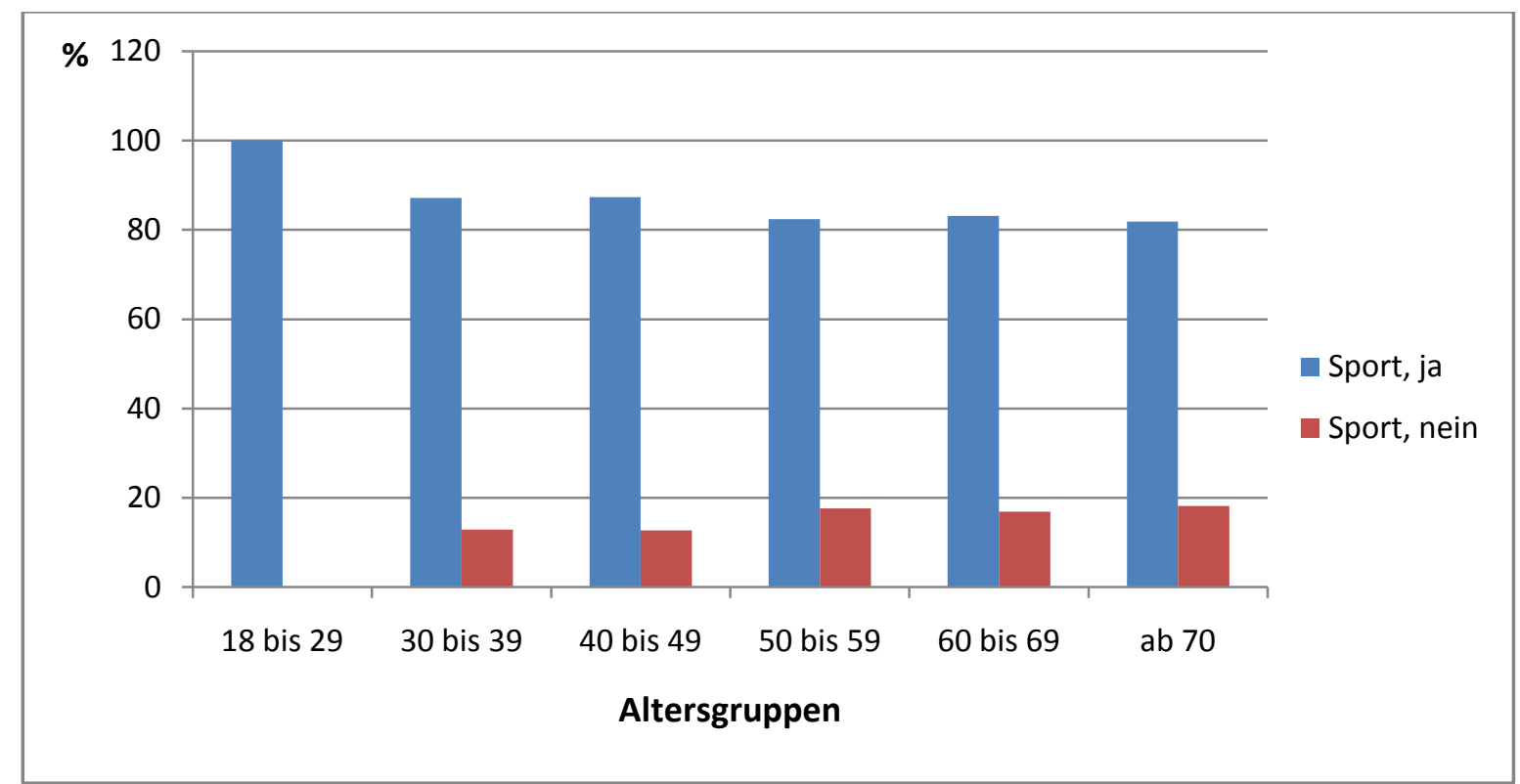

Abbildung 29: männliche Teilnehmer mit aktuellen Beschwerden (in \%), in den einzelnen Altersgruppen ( 18-29 Jahre, 3039 Jahre, 40-49 Jahre, 50-59 Jahre, 60-69 Jahre und ab 70 Jahre), stratifiziert nach dem Einflussfaktor Sport (betreiben von Sport und nicht betreiben von Sport)

Im Hinblick auf die Ergebnislage lässt sich nicht feststellen, ob der Sport positive oder negative Auswirkungen auf die muskuloskleletalen Beschwerden der Teilnehmer hat. Sport kann hier sowohl positive als auch negative Auswirkungen haben. „Ein abwechslungsreiches und vielseitiges Training, eine exakte Trainingsteuerung und Überwachung" und „sportmedizinische Voruntersuchungen auf Sporttauglichkeit“ (Beck et al. 2002, S. 410) sind beispielsweise Bedingungen, die sich vorteilhaft auf Beschwerden am muskuloskeletalen Bewegungsapparat auswirken können. Auf der anderen Seite ist es aber auch erwiesen, dass sportbedingte Überlastungen die Beschwerden hervorrufen oder negativ verstärken können (Beck et al. 2002). 
3.2.3. Zielgröße „aktuelle Beschwerden“ der Augenärzte/innen beeinflusst durch die Berufsjahre und deren Auswirkungen auf die Symptome und Lokalisationen der Beschwerden

Eine weitere Fragestellung ist, wie die Zielgröße „aktuelle Beschwerden“ durch die Berufsjahre der Augenärzte/innen beeinflusst wird. Man sieht sehr deutlich, dass mit steigenden Berufsjahren auch die Beschwerden häufiger werden. So sind in der Gruppe der $<10$ Berufsjahre 69\% $(n=67)$ der Teilnehmer mit aktuellen Beschwerden und 31\% $(n=30)$ haben keine Beschwerden, in der Gruppe der 10 - 20 Berufsjahre sind es 79\% ( $n=455)$ der Teilnehmer mit aktuellen Beschwerden und 21\% ( $n=121)$ ohne Beschwerden, in der Gruppe der 20 - 30 Berufsjahre sind es 83\% $(n=551)$ mit Beschwerden und 17\% ( $n=115)$ ohne Beschwerden, in der Gruppe der 30 - 45 Berufsjahre sind es 84\% ( $n=346)$ der Teilnehmer mit aktuellen Beschwerden und 16\% ( $n=66)$ haben keine Beschwerden. (s. Abb. 30)

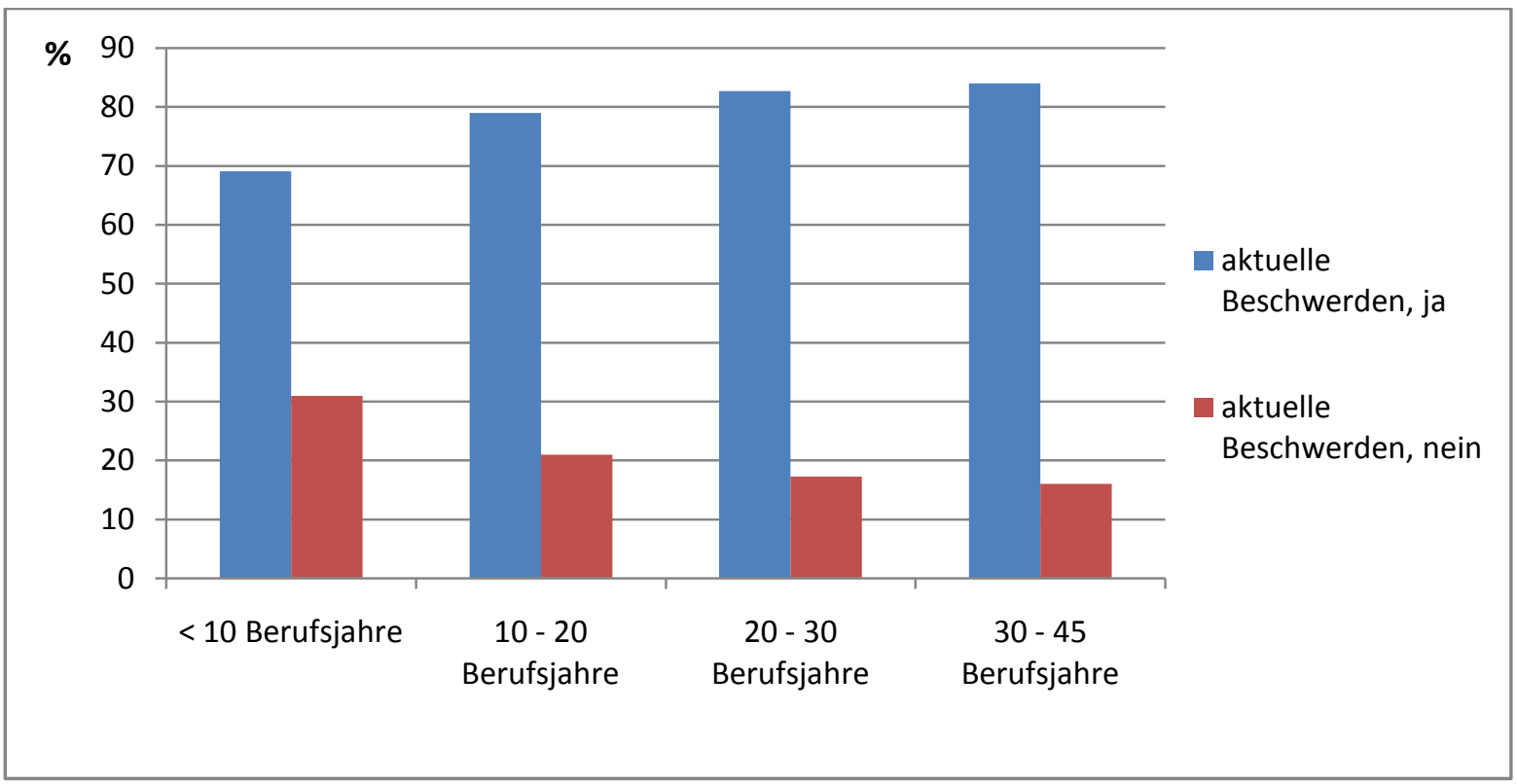

Abbildung 30: Augenärzte/innen mit aktuellen Beschwerden und ohne aktuelle Beschwerden (in \%) und deren Verteilung in den einzelnen Gruppen der Berufsjahre (<10 Berufsjahre, 10-20 Berufsjahre, 20-30 Berufsjahre, 30-45 Berufsjahre)

Bei der Gegenüberstellung der Berufsjahren und der bestehenden Symptomen in der Gruppe der Augenärzte/innen mit aktuellen Beschwerden zeigt sich, dass sich mit steigenden Berufsjahren auch die Symptome häufen. In den einzelnen Berufsgruppen haben die Teilnehmer mindestens 1 bis maximal 6 Symptome angegeben. Die meisten Symptome treten bei der Gruppe mit 20 - 30 Berufsjahren auf. In dieser Gruppe wurden dabei hauptsächlich die Symptome Verspannungen und Schmerzen genannt. Allerdings liegt auch bei den anderen Berufs - Gruppen der Schwerpunkt der berichteten Symptome bei den Verspannungen und Schmerzen. Die Symptome Parästhesien, Bewegungseinschränkung 
und Instabilität zeigt eine steigende Tendenz mit zunahme der Berufsjahre. Das Symptom Steifigkeit nimmt in den ersten drei Gruppen der Berufsjahren zu und es lies sich ein minimaler Abfall in der Berufsgruppe der 30 - 45 verzeichnen. Somit lässt sich festhalten, dass die Symptome mit steigendem Berufsjahren zunehmen. Was begründet sein kann zum einem in den zunehmenden degenerativen Veränderungen an der Wirbelsäule aber auch durch über Jahre durchgeführte unphysiologische Haltung bei den einzelnen Untersuchungsmethoden als Augenarzt. (s. Abb. 31)

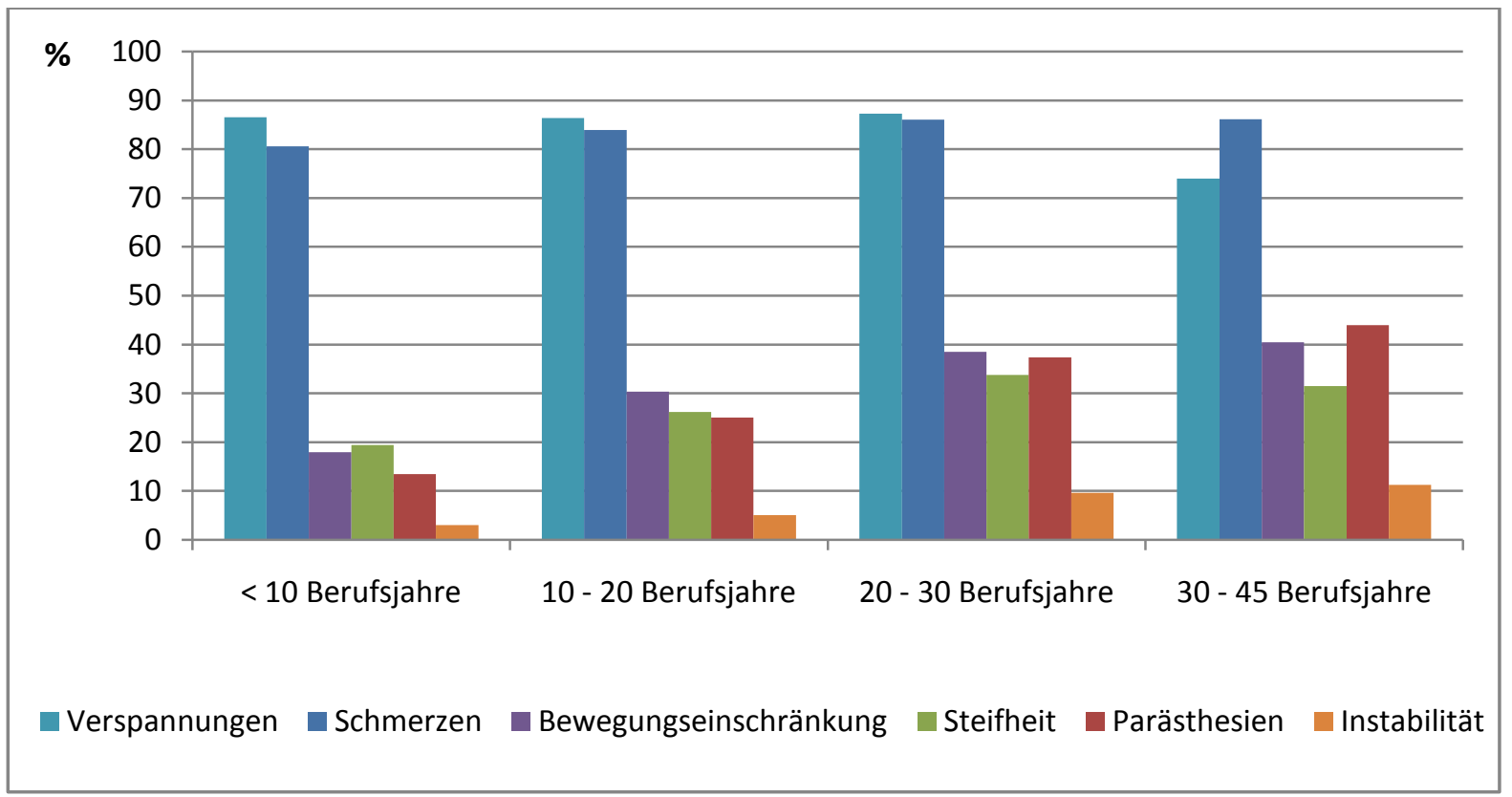

Abbildung 31: Augenärzte/innen mit aktuellen Beschwerden (in \%) (unter Berücksichtigung der Mehrfach-Antwort zu den Symptomen) und deren Verteilung in den einzelnen Gruppen der Berufsjahre (<10 Berufsjahre, 10-20 Berufsjahre, 20-30 Berufsjahre, 30-45 Berufsjahre), stratifiziert nach den Symptomen (Verspannungen, Schmerzen, Bewegungseinschränkungen, Steifheit, Parästhesien und Instabilität)

Die Auswertung der Beschwerdelokalisation im muskuloskeletalen Bewegungsapparat in Bezug auf die Gruppen der Berufsjahre der Teilnehmer mit aktuellen Beschwerden, wo bei auch hier mehrfach Antworten möglich waren. Ergibt ein ähnliches Bild, wie bei der Verteilung der Symptome in den einzelnen Gruppen der Berufsjahre, dass mit steigenden Berufsjahren die Häufigkeit der Beschwerden und damit die Häufigkeit der Lokalisationen der Beschwerden zunehmen. Die häufigsten Lokalisationen der Beschwerden sind bei allen vier Gruppen der Berufsjahre gleich: an erster Stelle stehen Beschwerden an der HWS, an zweiter Stelle kommen Beschwerden an der LWS, an dritter Stelle stehen die Beschwerden an der Schulter und an vierter Stelle die Beschwerden mit Ausstrahlungen in die obere Extremität mit muskulären Beschwerden. Bei den weniger häufigeren Lokalisationen der Beschwerden an der BWS, am ISG, an der oberen Extremität mit neurologischen 
Beschwerden, an der unteren Extremität muskulären Beschwerden und an der unteren Extremität mit neurologischen Beschwerden ist die Reihenfolge minimal unterschiedlich in den einzelnen Gruppen der Berufsjahre. (s. Abb. 32)

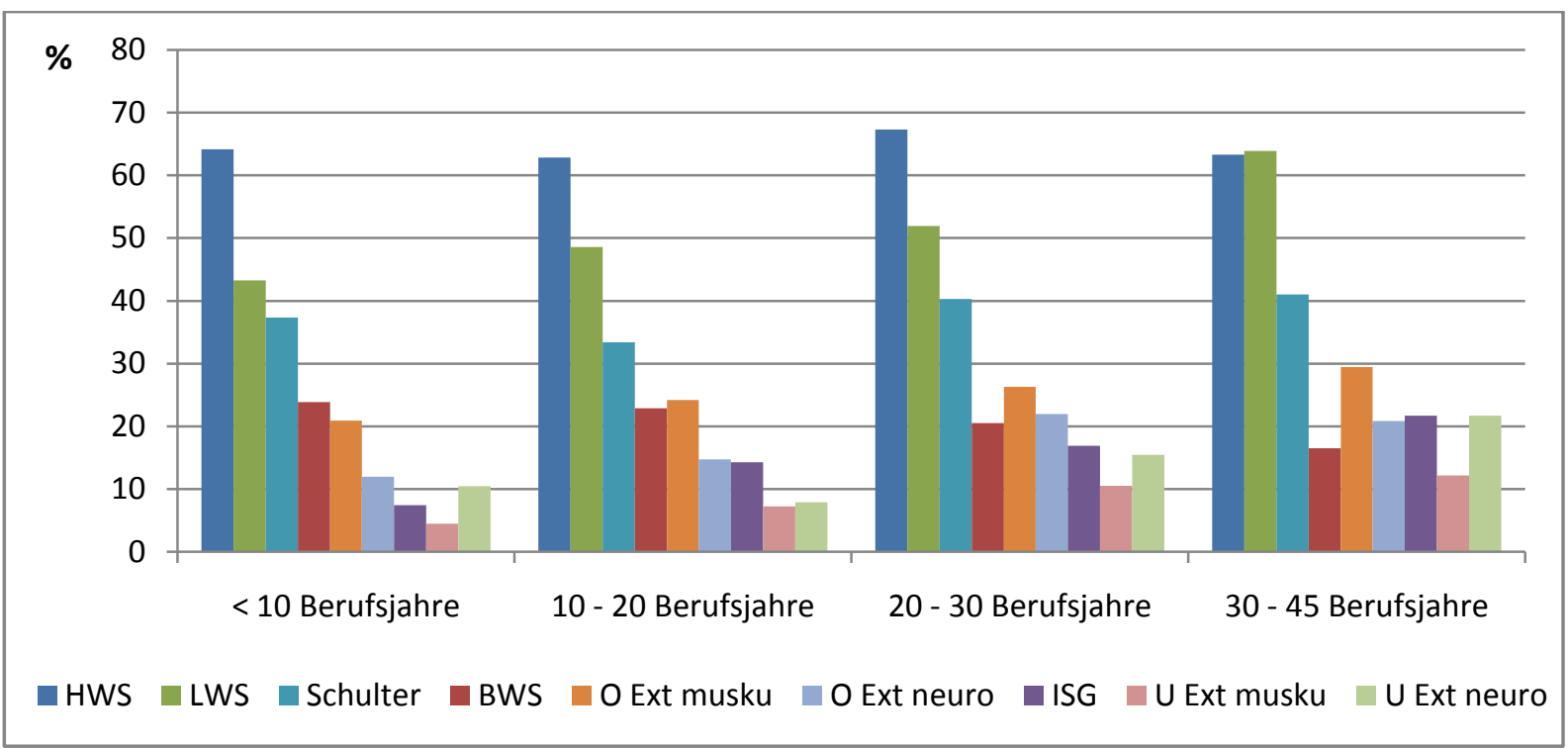

Abbildung 32: Augenärzte/innen mit aktuellen Beschwerden (in \%) (unter Berücksichtigung der Mehrfach-Antwort zu den Beschwerdelokalisationen) und deren Verteilung in den einzelnen Gruppen der Berufsjahre (<10 Berufsjahre, 10-20 Berufsjahre, 20-30 Berufsjahre, 30-45 Berufsjahre), stratifiziert nach den Beschwerdelokalisationen (Halswirbelsäule (HWS), Brustwirbelsäule (BWS), Lendenwirbelsäule (LWS), lleosakralgelenk (ISG), Schulter, obere Extremität mit muskulären Beschwerden (O Ext musku), obere Extremität mit neurologischen Beschwerden (O Ext neuro), untere Extremität mit muskulären Beschwerden (U Ext musku) und untere Extremität mit neurologischen Beschwerden (U Ext neuro)) 
3.2.4. Zielgröße „aktuelle Beschwerden“ der Augenärzte/innen beeinflusst durch die Tätigkeitsschwerpunkte „konservativ“, „operativ“ und Kombination von „konservativ“ und „operativ“ und deren Auswirkungen auf die Symptome und Lokalisationen der Beschwerden

Des Weiterem schauten wir, ob es einen Zusammenhang zwischen den Tätigkeitsschwerpunkten „konservativ“, „operativ“ und der Kombination von „konservativ“ und „operativ“ mit den bestehen aktuellen Beschwerden gibt. In allen Gruppen der Tätigkeitsschwerpunkte liegt der Prozentsatz der Augenärzte/innen weit über $70 \%$ mit aktuellen Beschwerden. Daraus ergibt sich eine Verteilung: Im „konservativen“ Bereich haben 79\% ( $n=448)$ der Teilnehmer aktuelle Beschwerden und 21\% ( $n=119)$ keine Beschwerden, im „konservativer > operativer" Breich sind es 83\% ( $n=743)$ der Teilnehmer mit aktuellen Beschwerden und 17\% ( $n=155)$ ohne Beschwerden, im „konservativer < operativer" Bereich sind es $81 \%(n=187)$ mit aktuellen Beschwerden und 19\% $(n=45)$ ohne Beschwerden und im „operativer" Bereich sind es 74\% ( $n=32)$ der Teilnehmer mit aktuellen Beschwerden und $26 \%(n=11)$ haben keine Beschwerden. (s. Abb. 33)

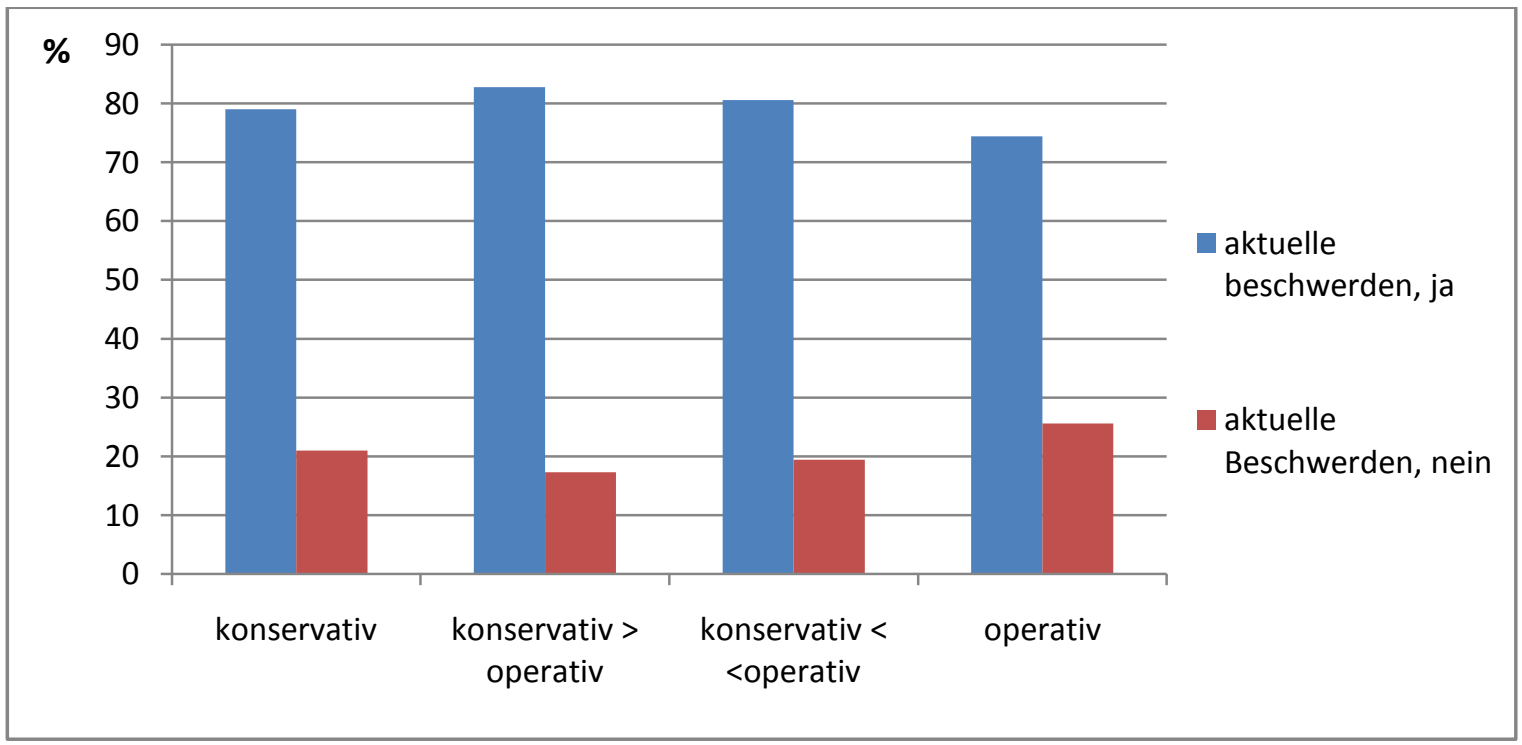

Abbildung 33: Augenärzte/innen mit aktuellen Beschwerden und ohne aktuelle Beschwerden (in \%) und deren Verteilung in den einzelnen Tätigkeitsschwerpunkten (rein konservativ, konservativ>operativ, konservativ<operativ und rein operativ)

Die Betrachtung der verschiedenen Tätigkeitsschwerpunkte erbrachte in Bezug auf die bestehenden Symptome, wobei die Augenärzte/innen mit aktuellen Beschwerden mindestens 1 bis maximal 6 Symptome angeben konnten, folgendes Ergebnis: in allen Tätigkeitsschwerpunkten sind die Symptome Schmerzen und Verspannungen am häufigsten und mit über $80 \%$ jeweils vertreten. 
Die Symptome Parästhesien, Steifheit, Bewegungseinschränkung und Instabilität des muskuloskeletalen Bewegungsapparates sind in den einzelnen Tätigkeitsschwerpunkten „konservativ“, „operativ“ und der Kombination von „konservativ“ und „operativ“ unterschiedlich vertreten. (s. Abb. 34)

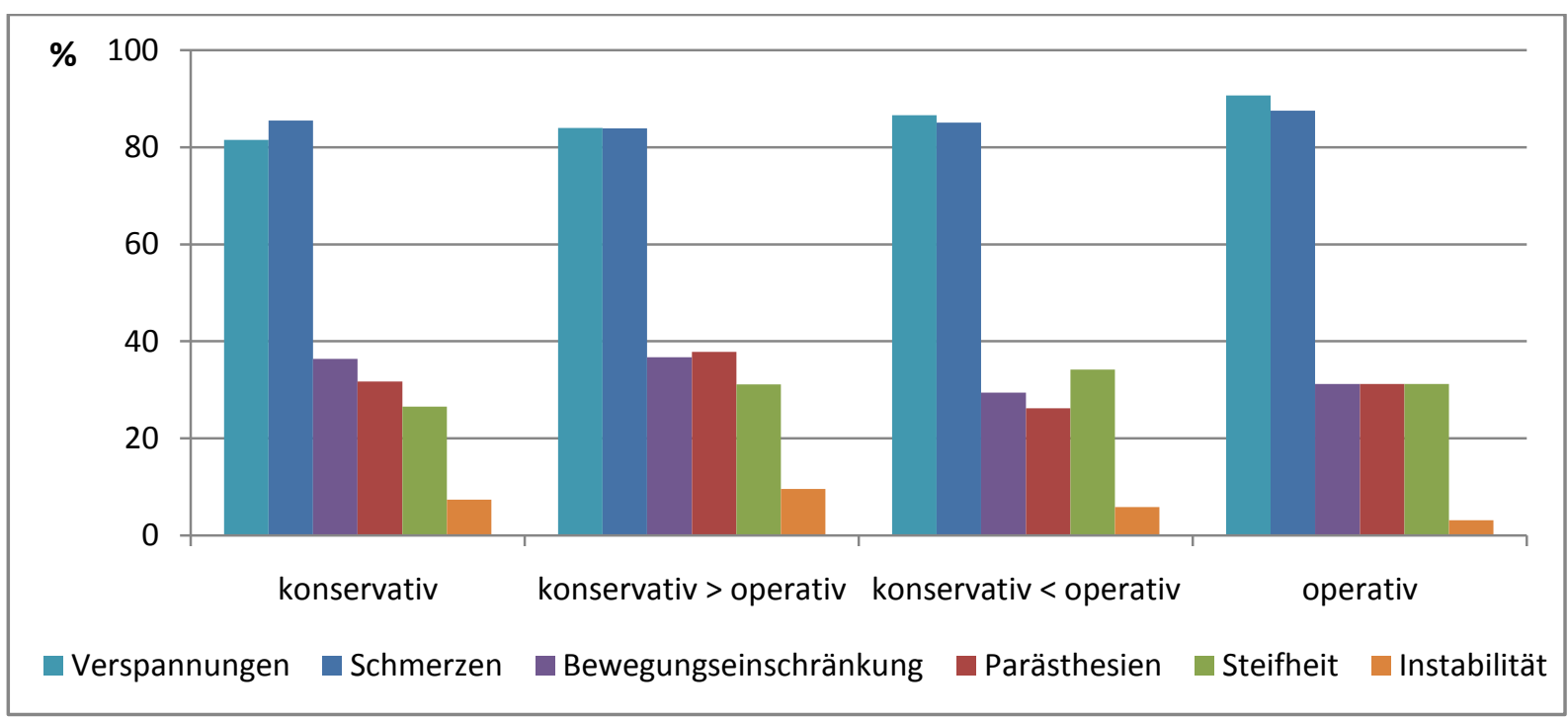

Abbildung 34: Augenärzte/innen mit aktuellen Beschwerden (in \%) (unter Berücksichtigung der Mehrfach-Antwort zu den Symptomen) und deren Verteilung in den einzelnen Tätigkeitsschwerpunkte (rein konservativ, konservativ>operativ, konservativ<operativ und rein operativ), stratifiziert nach den Symptomen (Verspannungen, Schmerzen, Bewegungseinschränkungen, Steifheit, Parästhesien und Instabilität)

Bei der Verteilung der Beschwerdelokalisation bezogen auf die Tätigkeitsschwerpunkte „konservativ", „operativ“ und der Kombination von "konservativ“ und "operativ“ in der Augenheilkunde ist zu erkennen, dass drei Bereiche besonders im Vordergrund stehen. Es bestand jedoch bei der Angabe der Beschwerdelokalisation die Möglichkeit der Mehrfach Antwort. Die Lokalisationen der Beschwerden am muskuloskeletalen Bewegungsapparates ist in den Tätigkeitsschwerpunkten „konservativ“ und „konservativ > operativ“ wie folgt: an erster Stelle stehen die Beschwerden an der HWS, an zweiter Stelle die Beschwerden an der LWS und an dritter Stelle die Beschwerden an der Schulter bei den Augenärzten/innen mit aktuellen Beschwerden.

Bei den Tätigkeitsschwerpunkten im „operativen“ Bereich und „konservativen < operativen“ Bereich stehen die Beschwerden der LWS an erster Stelle, gefolgt von den Beschwerden an der HWS. Die weitere Verteilung der anderen Beschwerden in den überwiegenden „operativen“ Tätigkeitsschwerpunkten ist sehr unterschiedlich. So stehen im „operativen“ Bereich die Beschwerden mit Ausstrahlungen in die obere Extremität mit muskulären Beschwerden und im „konservativen < operativen“ Bereich die Beschwerden an der Schulter dicht hinter den erst genannten. 
Die weiteren Lokalisationen der Beschwerden, wie an der BWS, am ISG, an der oberen Extremität mit neurologischen Beschwerden, an der unteren Extremität muskulären Beschwerden und an der unteren Extremität mit neurologischen Beschwerden ist die Reihenfolge minimal unterschiedlich in den einzelnen Tätigkeitsschwerpunkten in der Augenheilkunde, bei den Augenärzten/innen mit aktuellen Beschwerden. (s. Abb. 35)

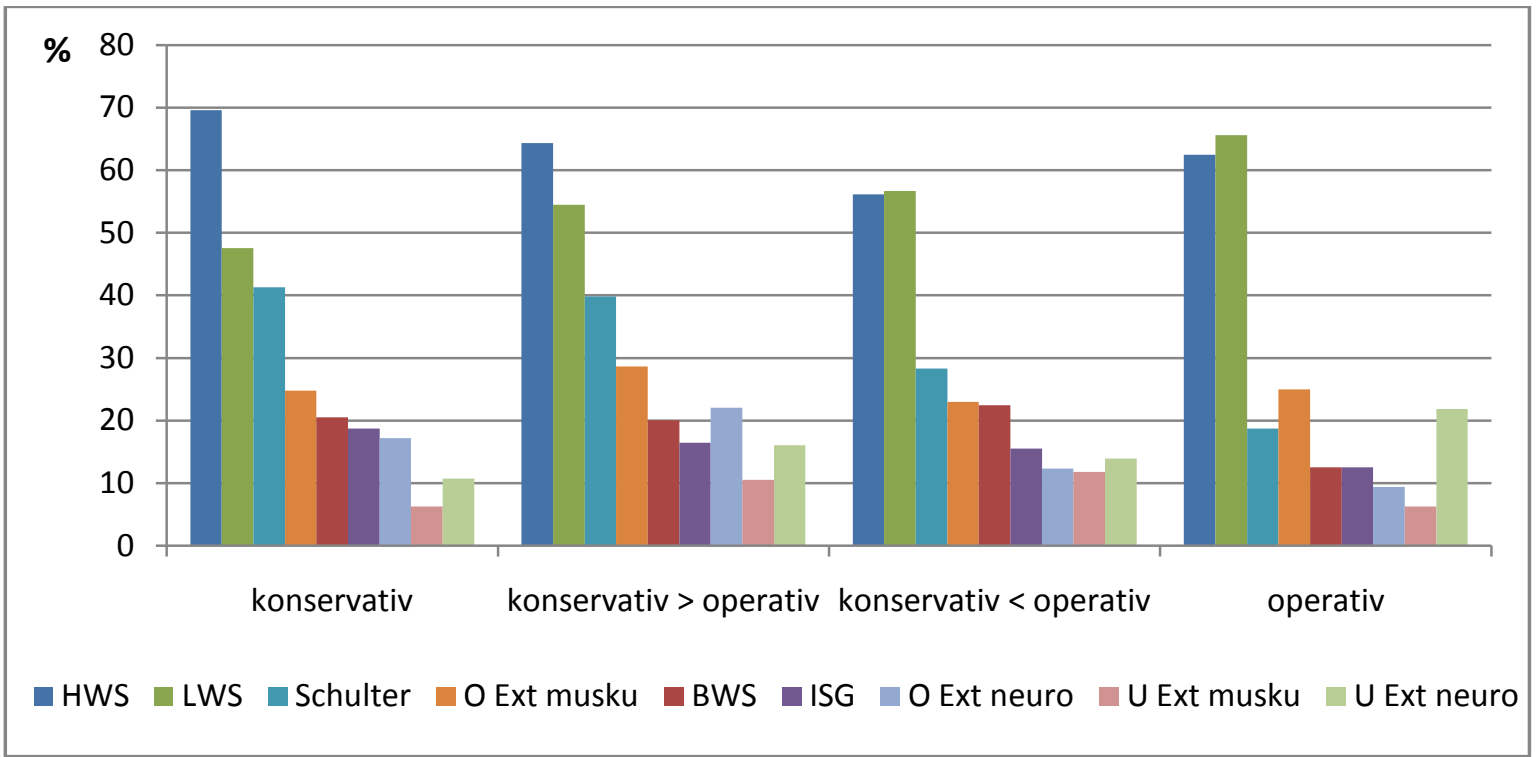

Abbildung 35: Augenärzte/innen mit aktuellen Beschwerden (in \%) (unter Berücksichtigung der Mehrfach-Antwort zu den Beschwerdelokalisationen) und deren Verteilung in den einzelnen Tätigkeitsschwerpunkte (rein konservativ, konservativ>operativ, konservativ<operativ und rein operativ), stratifiziert nach den Beschwerdelokalisationen (Halswirbelsäule (HWS), Brustwirbelsäule (BWS), Lendenwirbelsäule (LWS), lleosakralgelenk (ISG), Schulter, obere Extremität mit muskulären Beschwerden (O Ext musku), obere Extremität mit neurologischen Beschwerden (O Ext neuro), untere Extremität mit muskulären Beschwerden (U Ext musku) und untere Extremität mit neurologischen Beschwerden (U Ext neuro)) 


\subsection{Zielgröße „aktuelle Beschwerden“ und Einflußgrößen (Spezialgebiet,}

Tätigkeitsschwerpunkte, Sport) beeinflusst durch Alter und Geschlecht

Von den insgesamt 1464 Augenärzte/innen mit aktuellen Beschwerden, äußerten 56\% ( $n=827)$ der Teilnehmer die meisten Beschwerden bei der Tätigkeit an der Spaltlampe zu haben. Bei $15 \%(n=227)$ der Teilnehmer traten die Beschwerden hauptsächlich bei der Funduskopie auf, 10\% ( $n=150)$ geben Beschwerden bei der Arbeit am Schreibtisch an. 7\% $(n=104)$ der Teilnehmer gaben an, vornehmlich beim Operieren und 5\% $(n=70)$ der Teilnehmer berichteten vorwiegend beim Refraktionieren (Prüfung der Sehstärke) Beschwerden zu haben. 6\% $(n=86)$ der Teilnehmer machten hierzu keine Angaben (missing values). (s. Abb. 36)

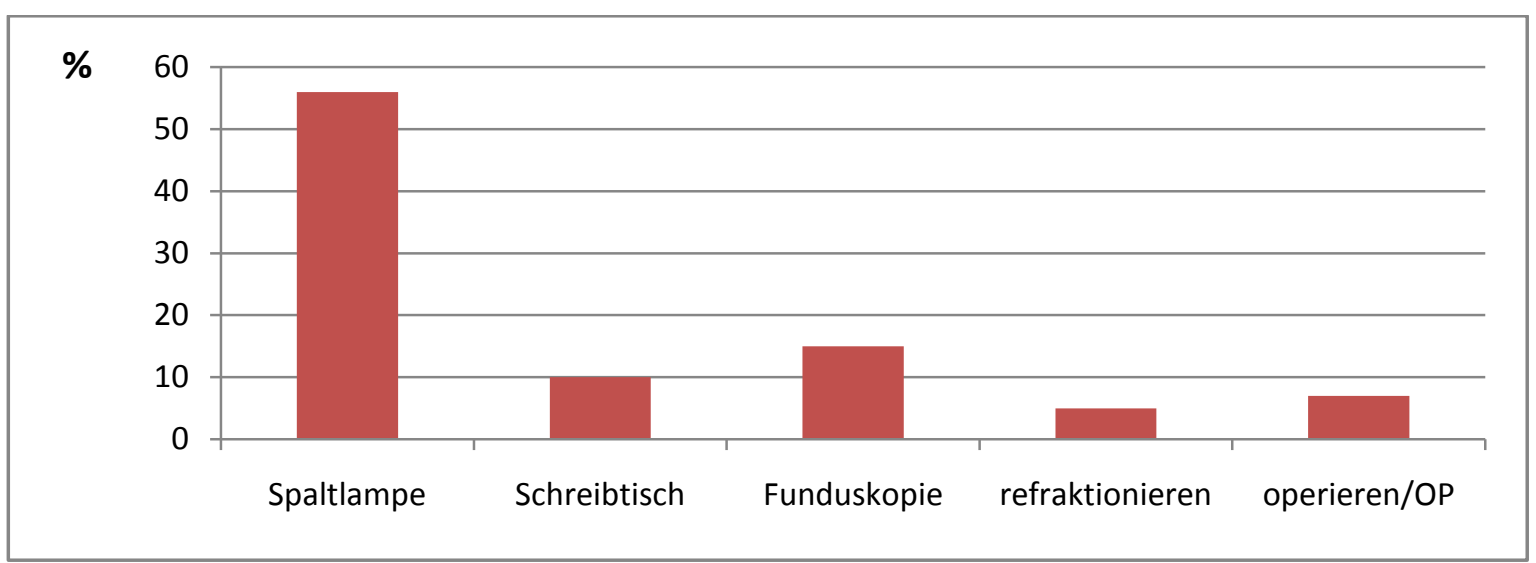

Abbildung 36: Darstellung der einzelnen augenärztlichen Tätigkeiten (Spaltlampe, Schreibtisch, Funduskopie, refraktionieren und operieren) und deren Verteilung unter den Augenärzten/innen mit aktuellen Beschwerden (in \%) 
3.4. Behandlungen, denen sich die Augenärzte/innen mit aktuellen Beschwerden am muskuloskeletalen Bewegungsapparat unterzogen

Die Augenärzte/innen mit aktuellen Beschwerden, konnten bei der Fragestellung nach den durchgeführten Behandlungen mehrere Antworten geben, was sich in der Gesamtsumme aller Behandlungen widerspiegelt.

$55 \%(n=807)$ der Teilnehmer führen ein Rückentraining durch, um ihre Beschwerden zu mindern, 53\% ( $n=782)$ nehmen Massagen in Anspruch, 47\% ( $n=694)$ gehen zur Krankengymnastik, 46\% ( $n=669)$ versuchen durch Medikamente ihre Beschwerden zu lindern. 43\% (n=636) führen Wärmebehandlungen durch, 14\% ( $n=205)$ nehmen osteopathische Behandlungen in Anspruch und 11\% ( $n=158)$ üben wegen ihrer Beschwerden Yoga aus. Bei 6\% $(n=85)$ der Teilnehmer mit aktuellen Beschwerden wurden Operationen durchgeführt. (s. Abb. 37)

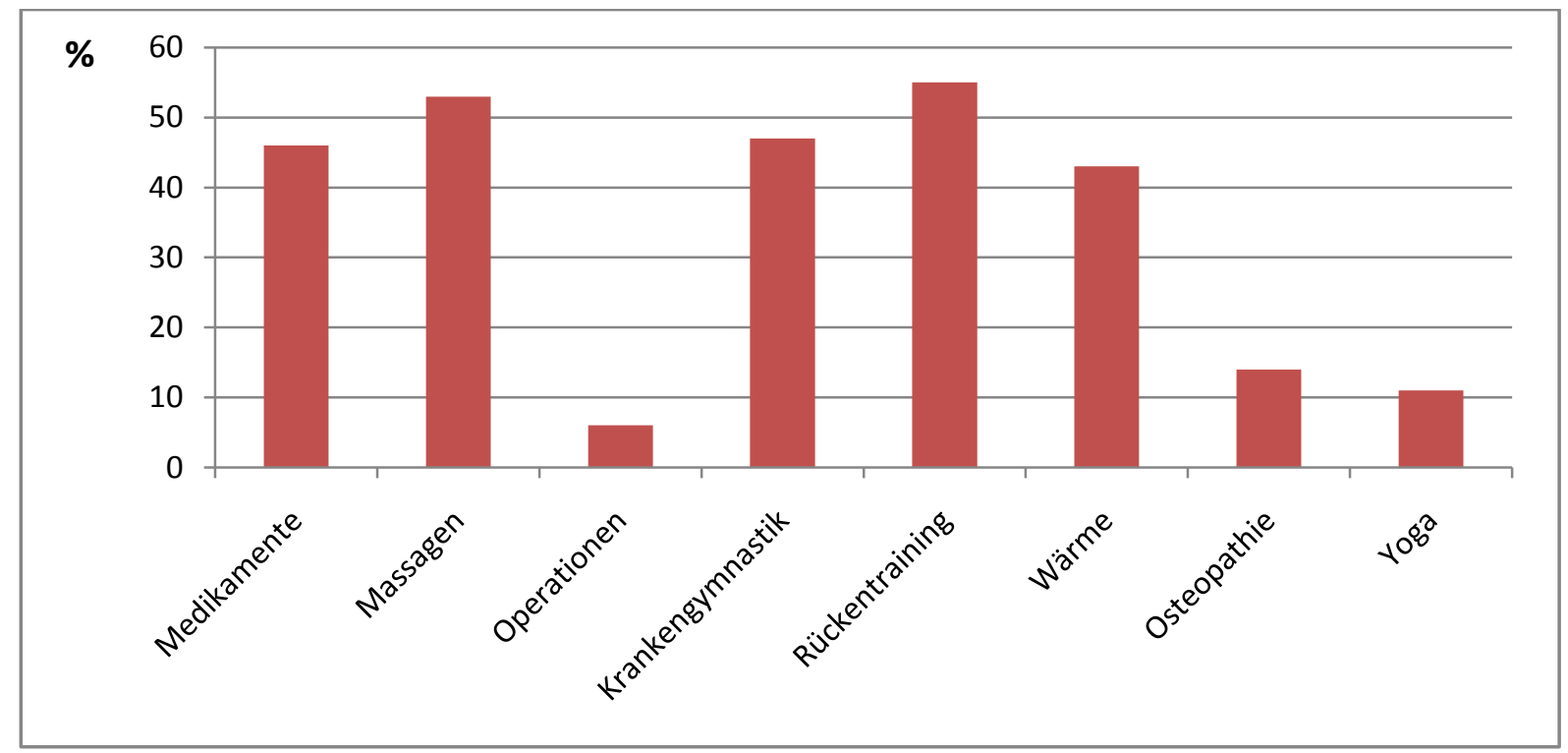

Abbildung 37: Augenärzte/innen mit aktuellen Beschwerden (in \%), stratifiziert nach durchgeführten Behandlungen (Medikamente, Massagen, Operationen, Krankengymnastik, Rückentraining, Wärme, Osteopathie und Yoga) 
3.5. Angaben aller Augenärzte/innen zur Intensität der Schmerzen/Beschwerden am muskuloskeletalen Bewegungsapparat

Alle Augenärzte/innen wurden zur Einstufung ihrer erlebten Schmerzen/Beschwerden mit Hilfe der Numerischen Analogskala (NAS) befragt. Diese Skala erstreckt sich von 0 (keine Schmerzen) bis 10 (unerträgliche Schmerzen). Im Mittel wurden die Schmerzen mit 3,6 bewertet. (s. Abb. 38)

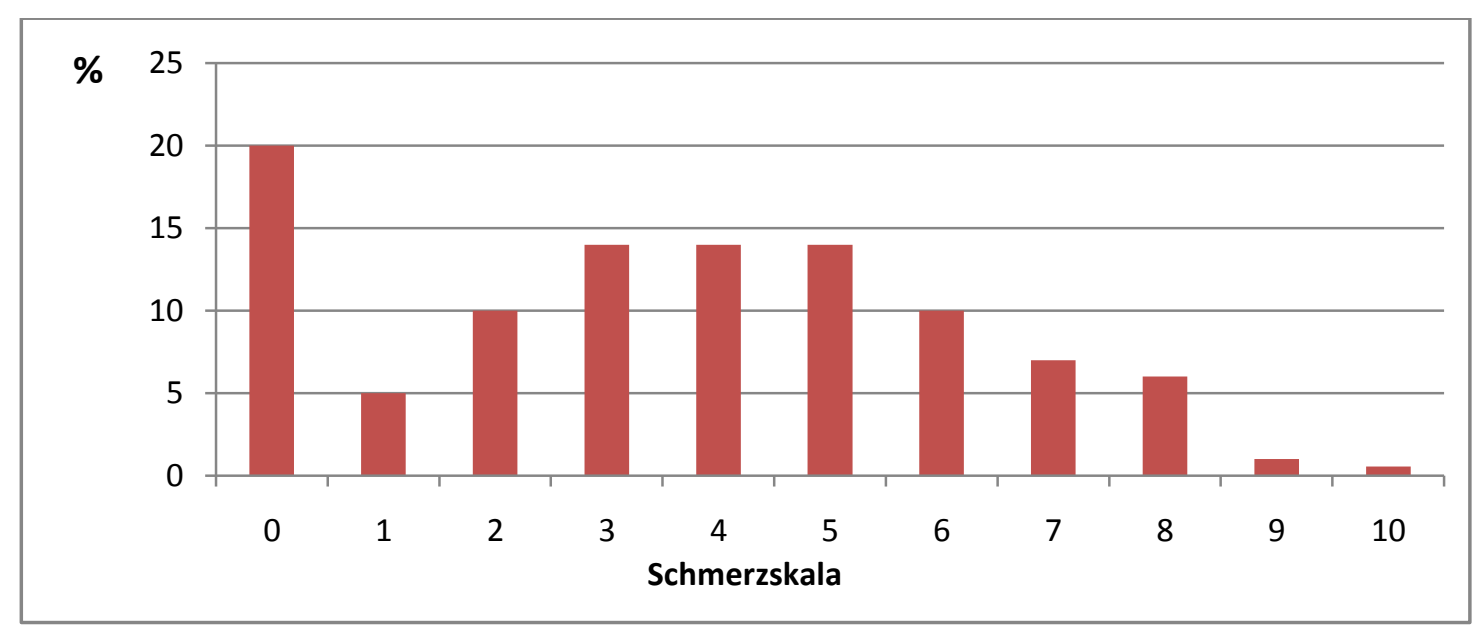

Abbildung 38: Einstufung der Intensität der Schmerzen/Beschwerden der Augenärzte/innen (in \%) auf der Numerischen Analogskala (0-keine Schmerzen bis 10-unerträgliche Schmerzen) 
3.6. Angaben zur Optimierung des Arbeitsplatzes in der Augenheilkunde

Eine Optimierung des Arbeitsplatzes halten 57\% ( $n=1038)$ der Augenärzte/innen für sinnvoll. Von denen befürworten sowohl $68 \%(n=708)$ der Teilnehmer eine höhenverstellbare Spaltlampen Einheit, als auch 57\% $(n=588)$ der Teilnehmer halten ein schwenkbares/abgewinkeltes Okular der Spaltlampe für sinnvoll.

Weitere Vorschläge zur Verbesserung des Arbeitsplatzes und der augenärztlichen Tätigkeit waren: ergonomische Sitzmöbel, ergonomisch zur Spaltlampe zugeordnete Schreibtische, Funduskopie am liegenden Patienten, wechselnde Anordnung des Schreibtisches rechts oder links der Spaltlampe um Einseitigkeit zu vermeiden, automatischer Phoropter, größere Beweglichkeit der Spaltlampe, bessere Fixation des Patientenkopfes und Positionierung des Patienten, Abstützung der Arme beim Funduskopieren und Operieren, Optimierung des OPTisches um mehr Beinfreiheit zu erreichen.

All diese Verbesserungsvorschläge zur Optimierung des Arbeitsplatzes und der augenärztlichen Tätigkeit könnten in Zusammenarbeit mit Herstellern ophthalmologischer Geräte als Grundlage für Verbesserungsmaßnahmen genutzt werden. 


\section{Diskussion}

4.1. Vergleich der Ergebnisse der Erhebung unter den Teilnehmern des BVA zu epidemiologischen Daten aus der Normalbevölkerung

Im Folgenden sollen die zuvor dargelegten Ergebnisse der Erhebung unter den Teilnehmern des BVA unter Hinzuziehung von Ergebnissen anderer Erhebungen in der Normalbevölkerung diskutiert werden.

Das zuvor bereits erwähnte Gesundheitssurvey des RKI und eine Studie der Betriebskrankenkassen Bundesverband (BKK BV) im November 2008 legen klar dar im Vergleich zu der Erhebung unter den Teilnehmern des BVA, dass insgesamt die Häufigkeit von bestehenden Rückenschmerzen unter den Augenärzten/innen wesentlich höher ist als in der Normalbevölkerung. Bei der Lokalisation der Beschwerden steht die LWS - Symptomatik in der Normalbevölkerung im Vordergrund, die Teilnehmer des BVA haben am häufigsten Beschwerden in der HWS dicht gefolgt von den Beschwerden in der LWS.

Das Gesundheitssurvey des RKI untersuchte die 12-Monatsprävalenz chronischer Rückenschmerzen (Neuhauser et al. 2005) innerhalb der Normalbevölkerung, auf die geschlechterspezifische Verteilung. $22 \%$ der befragten Frauen und $16 \%$ der Männer gaben dabei an, unter Rückenschmerzen zu leiden.

Ein Vergleich der Ergebnisse des Gesundheitssurveys des RKI mit den Ergebnissen der Teilnehmer des BVA zeigt, dass die Anteile der befragten Augenärzte/innen mit Beschwerden in allen Altersgruppen deutlich höher sind. So gaben beispielsweise in der Altersgruppe der 30- bis 39-Jährigen 69\% der Augenärztinnen an, von Beschwerden betroffen zu sein, während in dieser Altersgruppe nur 23\% der Normalbevölkerung Beschwerden bejahten. (s. Abb. 22) Ähnliches wurde auch bei der Auswertung der Daten der männlichen Teilnehmer deutlich. So steht hier beispielsweise ein Anteil von 69\% der 30 bis 39-jährigen Augenärzte mit Beschwerden einem Anteil von 15\% mit Beschwerden in der Normalbevölkerung gegenüber. (s. Abb. 23)

Darüber hinaus wurden die Befragten des bundesweiten telefonischen Gesundheitssurveys des RKI 2003 nach der Schmerzintensität mit Hilfe der NAS befragt. Der Median der Schmerzstärke auf einer Skala von 1 bis 10 betrug bei Frauen 5 und bei Männern 4 (Neuhauser et al. 2005). Bei den Teilnehmern des BVA liegt der Median der Schmerzstärke bei Frauen mit 3,8 und bei Männern mit 3,4 etwas tiefer, wobei es sich hier aber nicht um Befragte mit ausschließlich chronischen Beschwerden handelt. Nach beiden Umfragen lässt 
sich aber vermuten, das Frauen entweder stärker betroffen sind oder die Schmerzen intensiver und stärker wahrnehmen.

In einer der neuesten Umfragen zum Thema „Rückenschmerzen in der Allgemeinbevölkerung in Deutschland“, welche von dem BKK BV im November 2008 durchgeführt wurde, wurden 6013 Personen telefonisch über einen Zeitraum von 14 Jahren befragt. Aus dieser Langzeitstudie geht hervor, dass in den letzten zehn Jahren die Häufigkeit von Rückenschmerzen um eine Viertel gestiegen ist. Dabei zeigte sich, dass der Anteil der Personen, die täglich Rückenschmerzen haben, sich in den 10 Jahren von 1998 mit 6\% bis 2008 mit 15\% mehr als verdoppelt hat. Der Anteil der mehrmals pro Woche Betroffenen ist von $10 \%$ auf $16 \%$ angestiegen. Im Gegenzug ist der Anteil der seltener als einmal im Monat unter Schmerzen Leidenden leicht zurückgegangen (BKK BV 2008).

Des Weiteren wurde in der Befragung durch die BKK BV festgestellt: „Fast drei Viertel (73\%) der befragten Rückenschmerzpatienten gab 2008 an, dass bei ihnen der Schmerz im Lendenwirbelbereich sitzt." (BKK BV 2008, S.2). Im Gegensatz dazu ergab die Analyse der Ergebnisse aus der Erhebung unter den Teilnehmern des BVA, dass unter der Option der Mehrfachnennung 65\% ( $n=951)$ der befragten Augenärzte/innen die Beschwerden an der HWS lokalisierten und insgesamt 53\% ( $n=774)$ an der LWS. (s. Abb. 19)

Über die Ursache von Rückenschmerzen ergab die Befragung der BKK BV, dass „knapp ein Drittel der Betroffenen (29\%) [...] seine Rückenschmerzen auf Belastungen im Beruf“ (BKK BV 2008, S.2) zurück führt. Unter den 1807 Teilnehmern der BVA Mitgliedern, die derzeit muskuloskeletale Beschwerden haben, waren 71\% $(n=1037)$ der Meinung, dass diese Beschwerden auf die Tätigkeit als Augenarzt zurückzuführen sind. Dies ist ein deutlich höherer Anteil als bei der Befragung der BKK BV. 24\% ( $n=350)$ sehen die augenärztliche Tätigkeit als eine mögliche Ursache an und 4\% $(n=52)$ glauben nicht, dass ein Zusammenhang besteht. (s. Abb. 17)

Die Befragung der 6013 Personen der BKK BV nach den häufigsten Behandlungsmethoden für Rückenschmerzen ergab, dass im Jahr 2008 zwei Drittel der Patienten Medikamente gegen ihre Rückenschmerzen erhielten. „Zehn Jahre zuvor war es noch die Hälfte. Im ZehnJahres-Zeitraum ist die Verordnung von Krankengymnastik am stärksten gestiegen. Wurde diese 1998 in 22 Prozent der Fälle verordnet, so war die Krankengymnastik 2008 schon in 61 Prozent der Fälle die erste Wahl." (BKK BV 2008, S.3). Ein ähnliches Bild präsentiert sich in der Umfrage unter den Mitgliedern des BVA. Im Vordergrund der Behandlungsmethoden unter den Augenärzten/innen stehen Rückentraining mit 55\% 
$(n=807)$, Massagen mit 53\% $(n=782)$ und Krankengymnastik mit 47\% $(n=694)$. Die Behandlung mit Medikamenten führt mit 46\% ( $n=669)$ der Teilnehmer ein deutlich geringerer Anteil durch, dicht gefolgt von den Wärmebehandlungen bei $43 \%(n=636)$ der Teilnehmer und 11\% ( $n=158)$ üben wegen ihrer Beschwerden Yoga aus, 14\% ( $n=205)$ nehmen osteopathische Behandlungen in Anspruch und 6\% $(n=85)$ der Teilnehmer mit aktuellen Beschwerden unterzogen sich Operationen. (s. Abb. 37)

\subsection{Häufigkeit und Einfluss von Vorschäden auf bestehende Beschwerdelokalisationen}

Bei 18\% ( $n=325)$ der befragten Teilnehmer bestehen Vorschäden am muskuloskeletalen Bewegungsapparat. Unter den Teilnehmern mit Vorschäden haben $86 \%(n=278)$ aktuell Beschwerden.

Bei den Teilnehmern mit Vorschäden stellten sich drei dominierende Bereiche dar, wovon die Skoliose mit 38\% $(n=146)$ die häufigste Vorerkrankung darstellt, gefolgt vom Morbus Scheuermann mit 25\% ( $n=96)$ und dem Prolaps an der LWS mit 18\% ( $n=70)$. Die anderen Vorerkrankungen, wie der Prolaps an der HWS mit 7\% ( $n=27)$, BWS mit 5\% $(n=18)$ und dem ISG mit $2 \%(n=7)$, sowie das Trauma mit $6 \%(n=25)$ spielen eine untergeordnete Rolle. (s. Abb. 16)

In der Befragung der Augenärzte/innen wurde nicht im Speziellen darauf eingegangen, ob die Vorschäden mit den jetzigen Beschwerdelokalistionen in Verbindung stehen. Somit lässt sich nicht genau eruieren, ob zum Beispiel die Erkrankung mit Morbus Scheuermann eine tragende Rolle spielt für die jetzigen Beschwerden. Morbus Scheuermann ist eine Wachstumsstörung der jugendlichen Wirbelsäule, welche zu einer schmerzhaften Fehlhaltung führen kann. $4-6 \%$ der Gesamtbevölkerung sind von dieser Erkrankung betroffen (Andreae et al. 2008). Unter den Teilnehmern sind 5\% ( $n=96)$ vom Gesamtkollektiv betroffen. Die Erkrangung ist bei männlichen Jugendlichen doppelt so häufig als bei weiblichen Jugendlichen in der Normalbevölkerung. Die Wachstumsstörungen treten während des pubertären Wachstumsschubs auf, zwischen dem 10. und 15. Lebensjahr (Andreae et al. 2008). Bei der Betrachtung der 96 Augenärzte/innen mit Morbus Scheuermann, haben 82\% ( $n=79)$ aktuell Beschwerden. Die Geschlechterverteilung unter den Augenärzten/innen mit Morbus Scheuermann ist wie folgt: $72 \%(n=69)$ männliche und $26 \%(n=25)$ weibliche Teilnehmer und 2\% $(n=2)$ machten keine Angaben (missing values) zum Geschlecht. Somit liegt der Geschlechtsanteil der männlichen Teilnehmern über dem der Normalbevölkerung. Das Alter der Teilnehmer mit Morbus Scheuermann betrug zwischen 24 bis 38 Jahre. In der Verteilung der Beschwerdelokalisationen unter den 
Teilnehmern mit Morbus Scheuermann zeigt sich kein Unterschied in Bezug auf das Gesamtkollektiv der Augenärzte/innen.

\subsection{Einfluss der Berufsjahre}

In der Studie Back Pain Ophthalmologists (Chatterjee et al. 1994) wurde aufgezeigt, dass es einen Zusammenhang zwischen den geleisteten Berufsjahren und den Beschwerden gibt. Mit Zunahme der Berufsjahre steigt auch die Beschwerdehäufigkeit, das gleiche Bild ist auch unter den Mitgliedern des BVA zu beobachten.

Von den insgesamt 1807 Teilnehmern haben aktuell 81\% $(n=1464)$ Beschwerden an der Wirbelsäule und den Extremitäten. Erwartungsgemäß steigen die Beschwerden mit der Anzahl der Berufsjahre. Es ist bekannt, dass auch ohne zusätzliche Berufsbelastung degenerative Veränderungen an der Wirbelsäule schon im Alter von 20. bis 25 Jahren beginnen (Hasenbring 2003). Durch die jahrelang gleichbleibenden Bewegungsabläufe als konservativ und operativ tätiger Augenarzt können dennoch zusätzliche degenerative Veränderungen an der Wirbelsäule erwartet werden. So waren auch in dieser Umfrage die Gruppen mit 20 - 30 Berufsjahren und 30 - 45 Berufsjahren am häufigsten betroffen. Die Lokalisationen der aktuellen Beschwerden waren unabhängig von den Berufsjahren gleich verteilt: an erster Stelle standen Beschwerden an der HWS, an zweiter Stelle Beschwerden an der LWS, an dritter Stelle Beschwerden an der Schulter und an vierter Stelle Beschwerden mit Ausstrahlungen in die obere Extremität mit muskulären Beschwerden. (s. Abb. 32)

\subsection{Relevanz der Häufigkeit und Lokalisation der aktuellen Beschwerden in den einzelnen Tätigkeitsschwerpunkten}

Eine weitere Studie in der Augenheilkunde Symptoms of Musculoskeletal Disorders in Ophthalmologists (Dhimitri et al. 2005) untersuchte unter anderem die Lokalisation von Beschwerden im Nackenbereich, der oberen Extremität und im Bereich der Lendenwirbelsäule in der Allgemeinen Augenheilkunde und einzelnen Spezialisierungen der Augenheilkunde. In der Allgemeinen Augenheilkunde waren die Beschwerden am häufigsten an den oberen Extremitäten, gefolgt von den Beschwerden im Nackenbereich und den Beschwerden in der LWS. Im Vergleich zu den Mitgliedern des BVA stehen dort die Beschwerden an der HWS und LWS im Vordergrund, wobei die Allgemeine Augenheilkunde in „konservativ“ und „operativ“ untergliedert wurde. 
Die Mehrzahl der Augenärzte/innen äußerte sich, überwiegend „konservativ“ tätig zu sein. Die Lokalisationen der Beschwerden am muskuloskeletalen Bewegungsapparat ist in den Tätigkeitsschwerpunkten „konservativ“ und „konservativ > operativ“ wie folgt: An erster Stelle stehen die Beschwerden an der HWS, an zweiter Stelle die Beschwerden an der LWS und an dritter Stelle die Beschwerden an der Schulter.

In den Tätigkeitsschwerpunkten „operativ“ und „konservativ < operativ“ stehen die Beschwerden der LWS an erster Stelle, dicht gefolgt von den Beschwerden an der HWS. Die weitere Verteilung der anderen Beschwerden in den "operativen“ und „konservativen < operativen" Tätigkeitsschwerpunkten ist sehr unterschiedlich (s. Abb. 35).

Es lässt sich ein Zusammenhang zwischen den Tätigkeitsschwerpunkten „konservativ“, „operativ“ und der Kombination von „konservativ“ und „operativ“ und den Lokalisationen der Beschwerden am muskuloskeletalen Bewegungsapparat erkennen.

\subsection{Optimierung des Arbeitsplatzes}

Des Weiteren wurden in den Studien von Kohlmann und Schmidt (2005/2008) und Lühmann et al. (2003) epidemiologische Befunde aus bevölkerungsbezogenen Studien zu Riskofaktoren für die Entstehung und Persistenz von Rückenschmerzen aufgezeichnet. (s. Abb. 39) Es wurde das komplexe Zusammenspiel von verschiedenen Einflussfaktoren und deren Auswirkungen auf Rückenschmerzen dargestellt.

\begin{tabular}{|c|c|c|c|c|}
\hline Zusammenhang & Klinische Faktoren & Lebensstil,soziales Umfeld & Arbeitsplatzfaktoren & $\begin{array}{l}\text { Psychosoziale } \\
\text { Risikofaktoren }\end{array}$ \\
\hline gering & $\begin{array}{l}\text { - Übergewicht } \\
\text { - weibliches Geschlecht }\end{array}$ & $\begin{array}{l}\text { - Rauchen } \\
\text { - körperliche Inaktivität }\end{array}$ & & \\
\hline mittel & $\begin{array}{l}\text { schlechter subj. } \\
\text { Gesundheitzustand }\end{array}$ & $\begin{array}{l}\text { - geringes Einkommen } \\
\text { - niedrige Schicht } \\
\text { - niedrige Bildung }\end{array}$ & $\begin{array}{l}\text { - Arbeitspaltz Unzufriedenheit } \\
\text { - monotone Arbeit } \\
\text { - Stress } \\
\text { - geringe soziale Unterstützung } \\
\text { - Vibrationen } \\
\text { - schweres Heben } \\
\text { - ungünstige Körperhaltung }\end{array}$ & \multirow[t]{2}{*}{$\begin{array}{l}\text { - Katastrophisierung- } \\
\text { Fear-Avoidance } \\
\text { (Angst-Vermeidung) } \\
\text { - Somatisierung } \\
\text { - Depressivität } \\
\text { - Distress }\end{array}$} \\
\hline stark & $\begin{array}{l}\text { frühere Schmerzen in } \\
\text { anderen Körperregionen }\end{array}$ & & & \\
\hline sehr stark & frühere Rückenschmerzen & & & \\
\hline
\end{tabular}

Abbildung 39: Epidemiologische Befunde aus bevölkerungsbezogenen Studien zu Risikofaktoren für die Entstehung und Persistenz von Rückenschmerzen Quelle: Modifiziert aus Kohlmann und Schmidt (2005/2008), S.13

Es lassen sich Hinweise auf einen möglichen Zusammenhang zwischen Rückenschmerzen und verschiedenen sozialökonomischen und auf den Arbeitsplatz bezogenen Faktoren ableiten. Auch 57\% ( $n=1038)$ der Teilnehmer halten eine Optimierung des Arbeitsplatzes in ergonomischer Hinsicht zur Linderung der Beschwerden für sinnvoll. Weitere Anmerkungen 
der Teilnehmer zu auslösenden Faktoren der Beschwerden am muskuloskeletalen Bewegungsapparat waren unter anderem zu hohes Arbeitsaufkommen mit permanentem Druck bzw. Stress und zu wenig Bewegung während der Arbeit. Aus der oben genannten Erhebung können auch monotone Arbeit und ungünstige Körperhaltung für die Berufsgruppe der Augenärzte in Betracht gezogen werden.

Wie zum Beispiel: Im konservativen Bereich liegt der hauptsächliche Arbeitsschwerpunkt an der Spaltlampe (s. Abb. 1). Bei dieser Arbeit kommt es zu einem unphysiologischen Abknicken der HWS über mehrere Minuten pro Patient. Die Hersteller von Spaltlampen bieten bereits schwenkbare Okulare und auch höhenverstellbare Einheiten an, um die Untersuchungsposition zu berbessern.

Ein weiterer Faktor ist die Anzahl der durchgeführten Behandlungen pro Tag, sowie eine schlechte Haltung bei Arbeiten am Bildschirmarbeitsplatz, bei häufigem Refraktionieren am Phoropter, sowie beim Untersuchen der Netzhaut entweder mit dem Kontaktglas an der Spaltlampe oder mittels indirekter Ophthalmoskopie. Die dort verbundenen Zwangshaltungen verstärken die Probleme im HWS - Bereich. Erstaunlicherweise treten kaum Beschwerden im Schulter - Arm Bereich auf, obwohl dies beim Refraktionieren zu erwarten gewesen wäre. Einen Einfluss könnte der in vielen Augenarztpraxen bereits verbreitete automatische Phoropter haben.

Im operativen Arbeitsbereich stehen die Beschwerden an der LWS im Vordergrund, dicht gefolgt von den Beschwerden an der HWS. Wie in Abb. 2 zu erkennen, kommt es durch das bewegungsarme Verharren in ein und derselben Position am OP - Mikroskop, die teilweise Anspannung und die stärkere Beugung in der LWS zu verstärkten Beschwerden in diesem Bereich, aber auch das Blicken durch die Okulare führt zum Abknicken der HWS. Hier gibt es bereits von Seiten der Industrie ergonomische, aber teure Okularhalterungen für das Mikroskop, deren Anschaffung sich aber langfristig lohnt.

Die Teilnehmer dieser Studie haben viele Angaben zu den Verbesserungsvorschlägen für die Arbeitsgeräte gemacht sowie Hinweise zur ergonomischen Verbesserung des Arbeitsplatzes. Diese in Zusammenarbeit mit Herstellern ophthalmologischer Geräte gemachten Vorschläge zur Optimierung der Arbeitsplätze sollten als Grundlage für Verbesserungsmaßnahmen genutzt werden.

Des Weiteren sollten vor diesem Hintergrund die gesetzlichen Verpflichtungen des Arbeitsgebers in Bezug auf den Arbeitsschutz am Arbeitsplatz betrachtet werden. Die gesetzlichen Regelungen der Berufsgenossenschaften haben die vorrangige Aufgabe, den Arbeitgeber in Belangen von Arbeits- und Wegeunfällen, Berufskrankheiten und arbeitsbedingten Gesundheitsgefahren zu beraten, dieses in Bezug auf die Prävention arbeitsbedingter Erkrankungen. Der Arbeitgeber ist verpflichtet, erforderliche Maßnahmen 
zur Sicherheit und Gesundheit des Arbeitsnehmers am Arbeitsplatz durchzuführen und hat die Aufgabe, diese Maßnahmen auf Ihre Wirksamkeit hin zu überprüfen. Die Gesunderhaltung der Beschäftigten am Arbeitsplatz liegt dabei aber nicht nur in seiner Verpflichtung, sondern in Zeiten des zunehmenden Fachkräftemangels auch in seinem wirtschaftlichen Interesse. In Zusammenarbeit mit den Berufsgenossenschaften und den Arbeitgebern können die Ergebnisse Grundlage dafür sein, eine Diskussion zur Optimierung des Gesundheitsschutzes in der Augenheilkunde anzustoßen. 


\section{Zusammenfassung}

In der vorliegenden Arbeit wurde der Zusammenhang zwischen den Arbeitsbedingungen als Augenarzt und den daraus möglichen resultierenden Beschwerden an der Wirbelsäule und den Extremitäten untersucht. Da Beschwerden am muskuloskeletalen Bewegungsapparat zu den häufigsten gesundheitlichen Beschwerden in der Normalbevölkerung zählen, wurde im Rahmen der Arbeit ein Fragebogen erstellt und damit eine deutschlandweite Befragung durchgeführt, um an einem ausreichend großen Kollektiv mögliche spezifische Symptome, Beschwerden und Erkrankungen, verursacht durch die augenärztliche Tätigkeit, zu erkennen.

Von den insgesamt angeschriebenen 5954 Mitgliedern des BVA haben sich 30\% $(n=1807)$ an der Befragung beteiligt. Von den 1807 Teilnehmern gaben 81\% ( $n=1464)$ zur Zeit aktuell Beschwerden an der Wirbelsäule und den Extremitäten an.

Die Auswertung der Ergebnisse hat gerade auch vor dem Hintergrund von Vergleichen mit Daten aus der Normalbevölkerung im Rahmen des bundesweiten telefonischen Gesundheitssurveys des RKI ergeben, dass Augenärzte/innen insgesamt deutlich häufiger von Rückenschmerzen betroffen sind.

Hinsichtlich der Lokalisation der Beschwerden unter allen Augenärzten/innen werden an erster Stelle die HWS - Beschwerden genannt, gefolgt von den LWS- und den Schulter Beschwerden. Allerdings konnte hier in einer Gegenüberstellung gezeigt werden, dass andere Erhebungen in der Normalbevölkerung, in Bezug auf die Verteilung andere Resultate ergaben und diese daher von den Eigenschaften des Gesamtkollektivs abzuhängen scheinen. Bezüglich der Gruppen der Berufsjahre ist ersichtlich, dass mit steigenden Berufsjahren auch die Beschwerden zunehmen.

In Bezug auf die Tätigkeitsschwerpunkte „konservativ“, „operativ“ und die untersuchten Kombinationen von „konservativ“ und „operativ“ fällt auf, dass unter den Teilnehmern, die im konservativen Bereich tätig sind oder ihren Arbeitsschwerpunkt dort haben, die Beschwerden häufiger an der HWS lokalisiert wurden als an der LWS. Im operativen Bereich stellen sich die Beschwerdelokalisationen in umgedrehter Reihenfolge dar. Als eine mögliche Erklärung für diese Unterschiede in der Beschwerdelokalisation kann die unterschiedliche Arbeitshaltung in den Tätigkeitsbereichen „konservativ“ und „operativ“ gesehen werden.

Abschließend kann gesagt werden, dass sich in Bezug auf die bei Augenärzten/innen deutlich häufiger auftretenden Beschwerden im muskuloskeletalen Bereich ein Zusammenhang mit den Tätigkeitsschwerpunkten genauso wie mit den Berufsjahren der Augenärzte/innen erkennen lässt. 
Für eine Empfehlung, dass die Beschwerden als berufsbedingt anzuerkennen sind, entspricht die Datenlage nicht der bestehenden Definition der Berufskrankheit „Rückenschmerzen“. Dennoch lässt sich schon hier die Notwendigkeit zur Verbesserung der Arbeitsplätze im Sinne der Ergonomie erkennen, die auch von einem Großteil der Teilnehmer betont wurde. In Zusammenarbeit mit den Berufsgenossenschaften und den Arbeitgebern können die Ergebnisse Grundlage dafür sein, eine Diskussion zur Optimierung des Gesundheitsschutzes bei den Augenärzten/innen anzustoßen. 
6. Anhang: Fragebögen

Fragebogen 2006 und 2009

\section{Tagung der Retinologischen Gesellschaft 2006 in Kiel Die richtige Haltung als Augenarzt - Prophylaxe von Wirbelsäulenschäden}

\section{Umfrage unter den Mitgliedern des BVA 2006}
1. Ihr Alter Jahre
2. $\square$ Kollegin
$\square$ Kollege
keine Angabe

3. Welchen Arbeitsschwerpunkt in der Augenheilkunde haben Sie?

$\begin{array}{ll}\square \text { konservativ } & \square \quad \text { operativ } \\ \square \text { sonstiges } & \square \quad \text { keine Angabe }\end{array}$

beides

4. Wie lange arbeiten Sie bereits im augenärztlichen Fachgebiet?
$\square$ bis 10 Jahre
$\square$ 11-20 Jahre
$>20$ Jahre
im Ruhestand
keine Angabe

5. Wie/Wo sind Sie beschäftigt? (Falls zutreffend, mehrere Angaben möglich)

Art der Beschäftigung $\quad \square$ selbständig $\quad \square$ angestellt

sonstiges

Ort der Beschäftigung

Praxis

Krankenhaus

keine Angabe

sonstiges

keine Angabe

6. Sind bei Ihnen morphologische Schäden an der Wirbelsäule bekannt?

keine bekannt

degenerativ (Rö)

Bandscheiben-Vorfall

keine Angabe

7. Haben Sie Wirbelsäulenbeschwerden und wenn ja, welcher Art sind diese?

$\begin{array}{llllll}\text { Beschwerden } & \square & \text { ja } & \square \text { nein } & \square \text { intermittierend } \\ \text { Dauer der Beschwerden } & \square \text { seit kurzem } & \square<1 \text { Jahr } & \square \text { keine Angabe } \\ \text { Art der Beschwerden } & \square \text { akut } & \square \text { chronisch } & \square \text { J Jahr } \\ & & & \square \text { keine Angabe }\end{array}$

Falls Sie Frage 7 mit nein beantworten, bitte Fragen 8-12 auslassen und weiter mit Fragen 13-16

8. Wie sehen Ihre „Leitsymptome“ aus? (Falls zutreffend, mehrere Angaben möglich)
Schmerz
Parästhesien
Bewegungseinschränkung/Immobilität
Steifheit
andere

keine Angabe

9. Wo haben Sie am meisten Beschwerden? (Falls zutreffend, mehrere Angaben möglich)
$\square \quad$ HWS
$\square \quad$ Schult
$\square \quad$ LWS
$\square \quad$ obere Extremität
BWS
Schulter
$\square \quad$ untere Extremität
$\square \quad$ keine Angabe

10. Haben Sie wegen Ihrer Beschwerden eine Behandlung durchgeführt? (Falls zutreffend, mehrere Angaben möglich) 
bisher keine chiropraktisch

keine Angabe

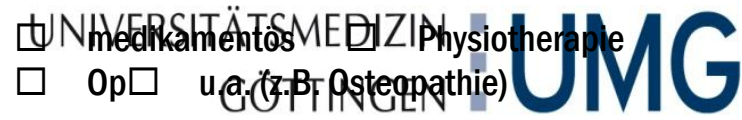

11. Welche Konsequenzen haben Sie angesichts Ihrer Beschwerden ergriffen? (Falls zutreffend, mehrere Angaben möglich)
$\square$ bisher keine
Physiotherapie
$\square \quad$ allg. Fitness/Sport
Analgetika bei Bed.
Rückentraining

\section{Wie bewerten Sie das Gesamtausmaß Ihrer Beschwerden?}
keine/leicht (,ich habe keine wesentliche Beeinträchtigung im Alltag“) moderat (,ich habe mich arrangiert, kann die Einschränkung kompensieren“)
schwer („erlebe ständige Einschränkung“)
sehr schwer („fürchte Berufsunfähigkeit“)
keine Angabe

13. Wie viele Patienten sehen Sie pro Woche?

weniger als 100

$\square \quad 100-300$

$>300$

keine Angabe

14. Haben Sie ein Spezialgebiet?

nein $\quad \square$ Vorderabschnitt

sonstiges (bitte spezifizieren)

Hinterabschnitt

keine Angabe

15. Führen Sie Laserbehandlungen durch?
$\square$ nein
$\square$ ja
is zu $20 /$ Woche
keine Angabe
Falls ja,
$\square$ bis zu 20/Woche
$\square>20 /$ Woche

16. Wie viele Operationen führen Sie pro Woche durch?
Katarakt
keine
bis zu 20
$>20 /$ Woche
keine Angabe
Netzhaut/Glaskörper OP
keine
bis zu 20
$>20 /$ Woche
keine Angabe
Lider
keine
bis zu 20
$>20 /$ Woche
keine Angabe
Sonstige
keine
bis zu 20
$>20 /$ Woche

Ggf. Kommentar:

$\odot$ Vielen Dank für Ihre Mitarbeit

S. Bopp ${ }^{1}$, L. Sagebiel ${ }^{2}$, C. Weiß ${ }^{3}$ und H. Hoerauf ${ }^{4}$

1 PD Dr. med. S. Bopp, Augenklinik Universitätsallee Bremen

2 L. Sagebiel, Physiotherapie und Physikalische Therapie, UK S-H, Campus Lübeck

3 Dr. med. C. Weiß, Koordinierungszentrum für klinische Studien Köln

${ }^{4}$ Prof. Dr. med. H. Hoerauf, Klinik für Augenheilkunde, UK S-H, Campus Lübeck

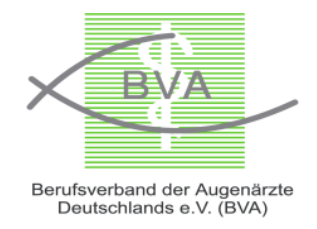


Universitäts-Augenklinik

Abteilung Augenheilkunde

z. Hd. Frau Silke Eichberg

Robert-Koch-Str. 40

37075 Göttingen

Fax: 0551-396787

\section{Umfrage unter den Mitgliedern des BVA 2009}

\section{Fragebogen: Muskuloskeletale Beschwerden unter Augenärzten}

1. Ihr Alter

Jahre

$\square$ w

$\mathrm{m}$

2. Haben Sie ein Spezialgebiet in der Augenheilkunde?
$\square$ nein
Strabologie
$\square$ Vorderabschnitt
Hinterabschnitt

3. In welchem Arbeitsbereich der Augenheilkunde arbeiten Sie heute vorwiegend?

\begin{tabular}{|l|l|l|l|l|l}
\hline & & & h/Woche konservativ und/oder $\square$ \\
\hline
\end{tabular} h/Woche operativ

a) Wie lange arbeiten Sie schon in der Augenheilkunde (mit Assistenzzeit)? gesamt $\square$ Jahre

davon: konservativ

Jahre und operativ

Jahre

4. Falls Sie aktuell operativ arbeiten, wie viele Operationen führen Sie typischer Weise pro Woche durch?

Katarakt

Netzhaut/Glaskörper OP

Lider/Strabismus

Laserbehandlungen

sonstige

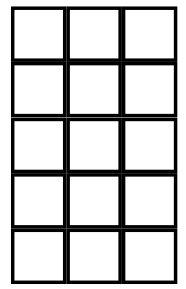

Anzahl

Anzahl

Anzahl

Anzahl

Anzahl

5. Gibt es Vorschäden an der Wirbelsäule, bevor Sie mit Ihrer Tätigkeit als Augenarzt (mit Assistenzzeit) begonnen haben?

$\square$ ja $\square$ nein

Falls ja,

a) ... welche Art/en von Vorschäden? (mehrere Antworten möglich)
Prolaps wo $\rightarrow$
$\square$ HWS
$\square$ BWS
$\square$ LWS
$\square$ Skoliose
M. Scheuermann
Trauma

ISG

6. Haben Sie aktuell Beschwerden im Bereich der WS oder/und Extremitäten?

$\square$ ja $\square$ nein

Falls ja,

a) Führen Sie diese Beschwerden auf Belastungen durch Ihre Tätigkeit als Augenarzt zurück?

$\square$ ja $\square$ nein $\square$ möglich

b) ... welche Symptome haben sie im Bereich der WS oder/und Extremitäten? (mehrere Antworten möglich)

$\square$ Schmerzen

Parästhesien

Steifheit 
$\square$ Bewegungseinschränkungen/Immobilität

Instabilität/Durchbrechgefühl

Verspannungen

c) ... wo haben Sie die meisten Beschwerden? (mehrere Antworten möglich)
HWS
$\square$ BWS
$\square$ LWS
$\square$ ISG
Schulter
Ausstrahlung in d. obere Extremität $\rightarrow \square$ muskulär oder $\square$ neurogen
Ausstrahlung in d. untere Extremität $\rightarrow \square$ muskulär oder $\square$ neurogen

d) Bei welcher Tätigkeit haben Sie die meisten Beschwerden?
$\square$ Spaltlampe
$\square$ Schreibtisch
Funduskopie
$\square$ refraktionieren
operieren/OP

e) Haben Sie wegen Ihrer Beschwerden eine Behandlung durchführen lassen oder selbst Heilverfahren eingeleitet? (mehrere Angaben möglich)
$\square$ Medikamente
Krankengymnastik
$\square$ Massagen
$\square$ OP
$\square$ Osteopathie
Rückentraining
Yoga
Wärme

7. Wie bewerten Sie das Gesamtausmaß Ihrer Beschwerden auf der Skala von 0 bis 10 ( 0 =keine Beschwerden, 10=ganz starke Beschwerden)?

8. Treiben/trieben Sie Sport?
$\square$ ja
$\square$ nein

Falls ja,

Aktuell: Wie viele

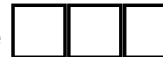
h/Woche und über wie viele Jahre

Früher: Wie viele h/Woche und über wie viele Jahre

9. Könnten Sie sich vorstellen, dass durch eine Optimierung Ihres Arbeitsplatzes eine Linderung der Beschwerden zu erzielen wäre?

$\square$ ja $\quad \square$ nein

a) Welche Veränderung/en finden Sie an Ihren Arbeitsplatz sinnvoll?

höhenverstellbare Spaltlampen - Einheit

schwenkbar/abgewinkelte Okulare für die Spaltlampe

sonstiges:

\section{Vielen Dank für Ihre Mitarbeit!}

Bei Rückfragen: - S. Eichberg, Universitätsmedizin Göttingen

Tel.: 0551- 2507059

E-Mail: s.eichberg@med.uni-goettingen.de

- Prof. Dr. med. H. Hoerauf , Augenklinik der Universitätsmedizin Göttingen Sekretariat:Tel.: 0551-39-67 76 Telefax: 0551-39-67 87

E-Mail: augenklinik@med-uni-goettingen.de

\section{Literaturverzeichnis}


(Al Wazzan et al. 2001) = Al Wazzan KA, Almas K, Al Shethri SE (2001): Rücken- und Nackenbeschwerden bei Zahnärzten und zahnmedizinischem Personal. J Contemp Dent Pract: (2)3:017-030

(Andreae et al. 2008) = Andreae S, Avelini P, Berg M: Lexikon der Krankheitem und Untersuchungen. 2. Auflage; Georg Thieme Verlag Stuttgart 2008

(Beck et al. 2002) = Beck H, Martin E, Motsch J, Schulte am Esch J, Kochs E, Krier C, Buzello W, Adams H A, van Aken H, Reinhardt K, Zimpfer M, Hempelmann G, Sefrin P: AINS-(Anästhesie-Intensivmedizin-Notfallmedizin-Schmerztherapie) Paket/Schmerztherapie: Band 4. 1. Auflage; Georg Thieme Verlag Stuttgart 2002

(BKK BV 2008) = Betriebskrankenkassen Bundesverband (2008): Langzeitstudie Rückenschmerzen. BKK Faktenspiegel November 2008;1-4

(Carey 1989) = Carey TS (1989): Occupational back pain: issues in prevention and treatment. Bailliére`s Clin Rheumatol;ㄹ: $143-56$

(Chatterjee et al. 1994) = Chatterjee A, Ryan WG, Rosen ES (1994): Back pain ophthalmologists. Eye; $\underline{8}: 473-474$

(Dhimitiri et al. 2005) = Dhimitiri KC, McGwin G, McNeal SF, et al. (2005): Symptoms of musculoskeletal disorders in ophthalmologists. Am J Ophthalmol 139:179-181

(Frank 1993) $=$ Frank A (1993): Low back pain. BMJ; $\underline{306}: 901-9$

(Hasenbring 2003) = Hasenbring M: Rückenschmerzen: Der Chronifizeirung frühzeitig entgegenwirken; in: Neuroorthopädie. Rückenschmerz interdisziplinär; hrsg. v. Casser HR, Forst R; Steinkopff Verlag Darmstadt 2003, 13-24

(Kohlmann und Schmidt 2005/2008) = Kohlmann T, Schmidt CO (2005/2008): Rückenschmerzen in Deutschland-ein epidemiologischer Überblick; in: Gesundheitsberichte spezial, Band 5: Rückengesundheit fördern und verbessern. Dokumentation der Fachtagung zu einem der zehn Gesundheitsziele im Land Nordrhein-Westfalen; hrsg. v. Ministerium für Arbeits, Gesundheit und Soziales des Landes Nordrhein-Westfalen; Düsseldorf 2008, 9-15 
(Lühmann et al. 2003) $=$ Lühmann D, Müller VE, Raspe H: Prävention von Rückenschmerzen. Expertise im Auftrag der Bertelsmann Stiftung und der Akademie für Manuelle Medizin, Universität Münster. Lübeck 2003

(Neuhauser et al. 2005) $=$ Neuhauser H, Ellert U, Ziese T (2005): Chronische Rückenschmerzen in der Allgemeinbevölkerung in Deutschland 2002/2003. Prävalenz der besonders betroffenen Bevölkerungsgruppen. Das Gesundheitswesen;10:685-93

(RKI 2006) = Robert-Koch-Institut (Hrsg): Gesundheit in Deutschland.

Gesundheitsberichterstattung des Bundes. Robert-Koch-Institut, Berlin 2006

(Seidel et al. 2005) = Seidel EJ, Fischer A, Seidel S, Zweiling K, Günzel W (2005): Belastungen der Wirbelsäule bei augenärztliche Tätigkeit. Der Augenarzt;모:205-207

(TKK 1997) = Techniker Krankenkasse (Hrsg): Rückenschmerzen. Eine Information für Patienten und Angehörige; 1. Auflage; Arcis Verlag GmbH, München/Düsseldorf 1997

(Weiß 2006/2008) = Weiß C: Koordinierungszentrum für klinische Studien Köln; SchriftlicheMitteilung 2008 über Daten von 2006 


\section{Danksagung}

Herrn Prof. Dr. med. H. Hoerauf möchte ich dafür danken, dass er mir das interessante Thema über muskuloskeletale Beschwerden unter Augenärzten überlassen hat. Seine gute Betreuung und seine vielen Anregungen haben maßgeblich zum Gelingen meiner Doktorarbeit beigetragen.

Frau PD Dr. A. Heutelbeck und Herrn Prof. E. Hallier, beide aus der Abteilung Arbeitsmedizin und Sozialmedizin der UMG, möchte ich danken für konstruktive Zusammenarbeit und Hilfestellung bei der Entwicklung des Fragebogens.

Auch Herrn Prof. Dr. R. Hilgers danke ich für seine konstruktive Kritik und seine vielen Ratschläge insbesondere beim Erstellen des Fragebogens und dessen Auswertung. Ein herzlicher Dank gilt ebenfalls den Mitarbeitern der Abteilung Medizinische Statistik der UMG, für die Unterstützung bei der Datenauswertung.

Außerdem möchte ich noch Frau B. Hartwig im Sekretariat der Augenheilkunde der UMG für Ihre Hilfsbereitschaft, Geduld, Freundlichkeit und Unterstützung im Hinblick auf die Rückführung der Fragebögen danken.

Des Weiteren bedanke ich mich bei Herrn Ch. Münscher aus der Abteilung Informationstechnologie Applikationen Forschung und Lehre der UMG, sowohl für die Erstellung der Online - Präsentation des Fragebogens als auch für die Erhebung der Online Daten.

Dem Berufsverband der Augenärzte Deutschlands (BVA) danke ich für die Verteilung des Fragebogens und der Firma bon Optik für die finanzelle Unterstützung dieser Studie.

Ebenso all denen ein Dankeschön, die hier nicht namentlich erwähnt sind, aber zur Entstehung dieser Dissertation beigetragen haben. 